Supporting Information

\title{
Selective Synthesis of Allylated Oxime Ethers and Nitrones Based on Palladium- Catalyzed Allylic Substitution of Oximes
}

Hideto Miyabe, Kazumasa Yoshida, Valluru Krishna Reddy, Akira Matsumura and Yoshiji Takemoto*

Graduate School of Pharmaceutical Sciences, Kyoto University, Yoshida, Sakyo-ku, Kyoto 606-8501, Japan.

FAX: +81-75-753-4569; E-mail: takemoto@pharm.kyoto-u.ac.jp

Table of contents

1. Experimental procedure: S1-2.

2. Characterization data of all obtained compounds: S2-8.

3. Copies of ${ }^{1} \mathrm{H}$ and ${ }^{13} \mathrm{C}$ NMR spectra of all obtained compounds: S8-67.

\section{Experimental procedure}

General. Melting points are uncorrected. ${ }^{1} \mathrm{H}$ and ${ }^{13} \mathrm{C}$ NMR spectra were recorded at 500 $\mathrm{MHz}$, and at $125 \mathrm{MHz}$, respectively. IR spectra were recorded using FTIR apparatus. Mass spectra were obtained by EI, CI or FAB methods. Preparative TLC separations were carried out on precoated silica gel plates $\left(\mathrm{E}\right.$. Merck $\left.60 \mathrm{~F}_{254}\right)$. E-Oximes were employed, after the separation of $E$ - and $Z$-isomers prepared from corresponding aldehydes and $\mathrm{HONH}_{2} \cdot \mathrm{HCl}$. Regarding the configurations of nitrones, $Z$-nitrone (major isomer) and $E$-nitrone (minor isomer) were obtained in all cases. These $E$ - and $Z$-isomers were easily separated by preparative TLC.

General procedure for palladium (0)-catalyzed reaction of $1 \mathrm{~A}$ with carbonate $2 \mathrm{a}$. A mixture of oxime $\mathbf{1 A}(60.6 \mathrm{mg}, 0.50 \mathrm{mmol})$, allylic carbonate $2 \mathbf{a}(144 \mathrm{mg}, 0.75 \mathrm{mmol})$, and $\mathrm{Pd}\left(\mathrm{PPh}_{3}\right)_{4}(23.1 \mathrm{mg}, 0.020 \mathrm{mmol})$ in the solvent $(2.5 \mathrm{~mL})$ shown in Table 1 was stirred under argon atmosphere at $20^{\circ} \mathrm{C}$. After the reaction was completed, the reaction mixture was concentrated at reduced pressure. The ratio of products and combined yield were determined after rough purification by column chromatography (hexame:AcOEt=1:1). Purification of the residue by preparative TLC (hexane:AcOEt=25:1, 2-fold development) afforded the products $\mathbf{3 A a - 5 A a}$.

General procedure for palladium (0)-catalyzed reaction of $1 \mathrm{~A}-\mathrm{G}$ with acetates in the presence of $\mathbf{E t}_{2} \mathbf{Z n}$. To a solution of oxime 1A-G $(0.50 \mathrm{mmol})$ in THF $(1.0 \mathrm{~mL})$ was added $\mathrm{Et}_{2} \mathrm{Zn}(1.0 \mathrm{M}$ in hexane, $0.50 \mathrm{~mL}, 0.50 \mathrm{mmol})$ under argon atmosphere at $20{ }^{\circ} \mathrm{C}$. After being stirred at the same temperature for $10 \mathrm{~min}$, a solution of allylic acetate $\mathbf{6 a - i}(0.75$ $\mathrm{mmol})$ and $\mathrm{Pd}\left(\mathrm{PPh}_{3}\right)_{4}(46.2 \mathrm{mg}, 0.040 \mathrm{mmol})$ in THF $(1.0 \mathrm{~mL})$ was added to the reaction 
mixture at $20^{\circ} \mathrm{C}$. After the reaction was completed, the reaction mixture was diluted with saturated aqueous potassium sodium $(+)$-tartrate and then extracted with AcOEt. The organic phase was dried over $\mathrm{MgSO}_{4}$ and concentrated at reduced pressure. Purification of the residue by preparative TLC (hexane:AcOEt=25:1, 2-fold development) afforded the product 3Aa-Ga.

General procedure for palladium (0)-catalyzed reaction of $1 \mathrm{~A}-\mathrm{G}$ with acetates in the presence of $\mathbf{K}_{2} \mathbf{C O}_{3}$. A mixture of oxime $\mathbf{1 A - G}(0.50 \mathrm{mmol})$, allylic acetate $\mathbf{6 a - f}(0.75$ $\mathrm{mmol}), \mathrm{Pd}\left(\mathrm{PPh}_{3}\right)_{4}(34.7 \mathrm{mg}, 0.030 \mathrm{mmol})$ and $\mathrm{K}_{2} \mathrm{CO}_{3}(69 \mathrm{mg}, 0.50 \mathrm{mmol})$ in $\mathrm{CH}_{2} \mathrm{Cl}_{2}(1.0$ $\mathrm{mL}$ ) was stirred under argon atmosphere at $20{ }^{\circ} \mathrm{C}$. After the reaction was completed, the reaction mixture was diluted with water and then extracted with AcOEt. The organic phase was dried over $\mathrm{MgSO}_{4}$ and concentrated at reduced pressure. Purification of the residue by preparative TLC (hexane:AcOEt=25:1, 2-fold development) afforded the product 3Aa-Ga.

General procedure for palladium (II)-catalyzed reaction of $1 \mathrm{~A}$ and $1 \mathrm{E}$ with acetates. A mixture of oxime $\mathbf{1 A}$ or $\mathbf{1 E}(0.50 \mathrm{mmol})$, allylic acetate $\mathbf{6 a - j}$ or $7(0.75 \mathrm{mmol}), \mathrm{Pd}(\mathrm{cod}) \mathrm{Cl}_{2}$ (14.3 mg, $0.050 \mathrm{mmol}$ ) was stirred under argon atmosphere at $90{ }^{\circ} \mathrm{C}$. After the reaction was completed, purification of the reaction mixture by preparative TLC (hexane:AcOEt=5:1, 2fold development) afforded the product 4Aa-Ga.

General procedure for palladium (II)-catalyzed reaction of 1 A with 7 using ligand 9. A mixture of oxime 1A $(33.5 \mathrm{mg}, 0.277 \mathrm{mmol})$, allylic acetate 7 (105 $\mathrm{mg}, 0.415 \mathrm{mmol})$, ligand $9(11.0 \mathrm{mg}, 0.0332 \mathrm{mmol}), \mathrm{Pd}(\operatorname{cod}) \mathrm{Cl}_{2}(7.9 \mathrm{mg}, 0.0277 \mathrm{mmol})$ was stirred under argon atmosphere at $30{ }^{\circ} \mathrm{C}$. After the reaction was stirred for $24 \mathrm{~h}$, purification of the reaction mixture by preparative TLC (hexane:AcOEt $=5: 1,2$-fold development) afforded the product 8 (59.1 mg, 68\% yield). Enantioselectivity of nitrone $\mathbf{8}$ was determined by HPLC analysis using Chiralcel AD-H (hexane/2-propanol=80/20, $0.5 \mathrm{ml} / \mathrm{min}, 254 \mathrm{~nm}, \mathrm{t}_{\mathrm{r}}=57.1$ and $63.2 \mathrm{~min}$.

\section{Characterization data of all obtained compounds}

(E)-O-(3-Phenylprop-2-enyl)benzaldehyde oxime (3Aa). ${ }^{1)}$ A colorless oil. IR $\left(\mathrm{CHCl}_{3}\right)$ 2926, 1494, $1448 \mathrm{~cm}^{-1} .{ }^{1} \mathrm{H}$ NMR $\left(\mathrm{CDCl}_{3}\right) \delta 8.13(1 \mathrm{H}, \mathrm{s}), 7.63-7.48(2 \mathrm{H}, \mathrm{m}), 7.46-7.16(8 \mathrm{H}$, m), $6.67(1 \mathrm{H}, \mathrm{d}, J=15.9 \mathrm{~Hz}), 6.42(1 \mathrm{H}, \mathrm{dt}, J=15.9,6.2 \mathrm{~Hz}), 4.83(2 \mathrm{H}, \mathrm{d}, J=6.2 \mathrm{~Hz}) .{ }^{13} \mathrm{C}$ NMR $\left(\mathrm{CDCl}_{3}\right) \delta 148.9,136.6,133.5,132.2,129.8,128.7,128.5,127.8,127.1,126.6$, 125.1, 74.9. MS $\left(\mathrm{EI}^{+}\right) \mathrm{m} / \mathrm{z}: 237\left(\mathrm{M}^{+}, 2\right), 117$ (100). HRMS calcd for $\mathrm{C}_{16} \mathrm{H}_{15} \mathrm{NO}: 237.1154$, Found: 237.1155 .

(E)-O-[3-(4-Trifluoromethylphenyl)prop-2-enyl]benzaldehyde oxime (3Ab). A colorless crystal. mp 55-58 ${ }^{\circ} \mathrm{C}$ (hexane). IR $\left(\mathrm{CHCl}_{3}\right) 2926,1492,1447,1415 \mathrm{~cm}^{-1} .{ }^{1} \mathrm{H} \mathrm{NMR}\left(\mathrm{CDCl}_{3}\right)$ d $8.15(1 \mathrm{H}, \mathrm{s}), 7.65-7.55(4 \mathrm{H}, \mathrm{m}), 7.49(2 \mathrm{H}, \mathrm{d}, J=7.9 \mathrm{~Hz}), 7.40-7.36(3 \mathrm{H}, \mathrm{m}), 6.71(1 \mathrm{H}, \mathrm{d}$, $J=16.2 \mathrm{~Hz}), 6.50(1 \mathrm{H}, \mathrm{dt}, J=16.2,6.1 \mathrm{~Hz}), 4.86(2 \mathrm{H}, \mathrm{d}, J=6.1 \mathrm{~Hz}) .{ }^{13} \mathrm{C} \mathrm{NMR}\left(\mathrm{CDCl}_{3}\right) \delta$ $149.2,140.2$, 132.1, 131.6, 130.0, 129.5 (q, $J=32 \mathrm{~Hz}), 128.7,128.1,127.1,126.7,125.5$ (q, 
$J=4.1 \mathrm{~Hz}), 124.2(\mathrm{q}, J=272 \mathrm{~Hz}), 74.4 . \mathrm{MS}\left(\mathrm{EI}^{+}\right) \mathrm{m} / \mathrm{z}: 305\left(\mathrm{M}^{+}, 2\right), 185$ (100). HRMS calcd for $\mathrm{C}_{17} \mathrm{H}_{14} \mathrm{~F}_{3} \mathrm{NO}$ : 305.1027, Found: 305.1025. Anal. Calcd for $\mathrm{C}_{17} \mathrm{H}_{14} \mathrm{~F}_{3} \mathrm{NO}$ : C, 66.88; $\mathrm{H}$, 4.62; N, 4.59; F, 18.67. Found: C, 66.65; H, 4.73; N, 4.44; F, 18.96.

(E)-O-[3-(4-Methoxyphenyl)prop-2-enyl]benzaldehyde oxime (3Ac). A colorless oil. IR $\left(\mathrm{CHCl}_{3}\right) 2935,1512,1464,1445 \mathrm{~cm}^{-1} .{ }^{1} \mathrm{H} \mathrm{NMR}\left(\mathrm{CDCl}_{3}\right)$ \& $8.14(1 \mathrm{H}, \mathrm{s}), 7.63-7.57(2 \mathrm{H}, \mathrm{m})$, 7.40-7.28 (5H, m), $6.84(2 \mathrm{H}, \mathrm{d}, J=8.6 \mathrm{~Hz}), 6.63(1 \mathrm{H}, \mathrm{d}, J=15.9 \mathrm{~Hz}), 6.29(1 \mathrm{H}, \mathrm{dt}, J=15.9$, $6.5 \mathrm{~Hz}), 4.81(2 \mathrm{H}, \mathrm{d}, J=6.5 \mathrm{~Hz}), 3.78(3 \mathrm{H}, \mathrm{s}) .{ }^{13} \mathrm{C} \mathrm{NMR}\left(\mathrm{CDCl}_{3}\right) \delta 159.4,148.8,133.3$, 132.3, 129.8, 129.4, 128.7, 127.8, 127.1, 122.7, 113.9, 75.1, 55.2. MS (EI $)$ m/z: $267\left(\mathrm{M}^{+}\right.$, 6), 147 (100). HRMS calcd for $\mathrm{C}_{17} \mathrm{H}_{17} \mathrm{NO}_{2}$ : 267.1259, Found: 267.1253 .

(E)-O-[3-(1-Naphtyl)prop-2-enyl]benzaldehyde oxime (3Ad). A colorless oil. IR $\left(\mathrm{CHCl}_{3}\right) 2925,1507,1493,1446 \mathrm{~cm}^{-1} .{ }^{1} \mathrm{H}$ NMR $\left(\mathrm{CDCl}_{3}\right) \delta 8.17(1 \mathrm{H}, \mathrm{s}), 8.12(1 \mathrm{H}, \mathrm{d}, J=7.9$ $\mathrm{Hz}), 7.82(1 \mathrm{H}, \mathrm{d}, J=6.4 \mathrm{~Hz}), 7.75(1 \mathrm{H}, \mathrm{d}, J=8.2 \mathrm{~Hz}), 7.68-7.55(3 \mathrm{H}, \mathrm{m}), 7.51-7.30$ (7H, m), $6.44(1 \mathrm{H}, \mathrm{dt}, J=15.8,6.1 \mathrm{~Hz}), 4.94(2 \mathrm{H}, \mathrm{d}, J=6.1 \mathrm{~Hz}), 3.78(3 \mathrm{H}, \mathrm{s}) .{ }^{13} \mathrm{C} \mathrm{NMR}\left(\mathrm{CDCl}_{3}\right) \delta$ $149.0,134.5,133.6,132.3,131.2,130.6,129.9,128.7,128.5,128.4,128.1,127.1,126.1$, 125.8, 125.6, 124.1, 123.8, 75.0. MS (EI $\left.{ }^{+}\right) \mathrm{m} / \mathrm{z}: 287\left(\mathrm{M}^{+}, 10\right), 167$ (100). HRMS calcd for $\mathrm{C}_{20} \mathrm{H}_{17} \mathrm{NO}: 287.1310$, Found: 287.1311 .

(E)-O-[3-(2-Naphtyl)prop-2-enyl]benzaldehyde oxime (3Ae). A colorless crystal. mp 70$73{ }^{\circ} \mathrm{C}$ (hexane). IR $\left(\mathrm{CHCl}_{3}\right) 2927,1506,1446 \mathrm{~cm}^{-1} .{ }^{1} \mathrm{H} \mathrm{NMR}\left(\mathrm{CDCl}_{3}\right) \delta 8.16(1 \mathrm{H}, \mathrm{s}), 7.83-$ $7.75(4 \mathrm{H}, \mathrm{m}), 7.66-7.58(3 \mathrm{H}, \mathrm{m}), 7.49-7.34(5 \mathrm{H}, \mathrm{m}), 6.85(1 \mathrm{H}, \mathrm{d}, J=15.9 \mathrm{~Hz}), 6.56(1 \mathrm{H}, \mathrm{dt}$, $J=15.9,6.1 \mathrm{~Hz}), 4.90(2 \mathrm{H}, \mathrm{d}, J=6.1 \mathrm{~Hz}) .{ }^{13} \mathrm{C} \mathrm{NMR}\left(\mathrm{CDCl}_{3}\right) \delta 149.0,134.1,133.6,133.1$, 132.2, 129.9, 128.7 (2C), 128.2, 128.0, 127.7, 127.1, 126.7, 126.3, 126.0, 125.5, 123.6, 75.0. MS (EI $\left.{ }^{+}\right) \mathrm{m} / \mathrm{z}: 287\left(\mathrm{M}^{+}, 6\right), 167$ (100). HRMS calcd for $\mathrm{C}_{20} \mathrm{H}_{17} \mathrm{NO}: 287.1310$, Found: 287.1312. Anal. Calcd for $\mathrm{C}_{20} \mathrm{H}_{17} \mathrm{NO}$ : C, 83.59; H, 5.96; N, 4.87. Found: C, 83.72; H, 5.97; N, 4.83 .

(E)-O-[3-(4-Fluorophenyl)prop-2-enyl]benzaldehyde oxime (3Af). A colorless crystal. mp 54-57 ${ }^{\circ} \mathrm{C}$ (hexane). IR $\left(\mathrm{CHCl}_{3}\right) 2926,1509,1447,1414 \mathrm{~cm}^{-1} .{ }^{1} \mathrm{H}$ NMR $\left(\mathrm{CDCl}_{3}\right) \delta 8.14$ $(1 \mathrm{H}, \mathrm{s}), 7.63-7.57(2 \mathrm{H}, \mathrm{m}), 7.42-7.34(5 \mathrm{H}, \mathrm{m}), 7.05-6.97(2 \mathrm{H}, \mathrm{m}), 6.65(1 \mathrm{H}, \mathrm{d}, J=15.8 \mathrm{~Hz})$, $6.34(1 \mathrm{H}, \mathrm{dt}, J=15.8,6.1 \mathrm{~Hz}), 4.82(2 \mathrm{H}, \mathrm{d}, J=6.1 \mathrm{~Hz}) .{ }^{13} \mathrm{C} \mathrm{NMR}\left(\mathrm{CDCl}_{3}\right) \delta 162.5(\mathrm{~d}, J=247$ $\mathrm{Hz}$ ), 149.0, 132.8, 132.3, 132.2, 129.8, 128.7, 128.1 (d, J=8.3 Hz), 127.1, 124.9, 115.4 (d, $J=21.7 \mathrm{~Hz}), 74.7$. MS $\left(\mathrm{EI}^{+}\right) \mathrm{m} / \mathrm{z}: 255\left(\mathrm{M}^{+}, 1\right), 135$ (100). HRMS calcd for $\mathrm{C}_{16} \mathrm{H}_{14} \mathrm{FNO}$ : 255.1059, Found: 255.1062. Anal. Calcd for $\mathrm{C}_{16} \mathrm{H}_{14} \mathrm{FNO}$ : C, 75.28; H, 5.53; N, 5.49; F, 7.44. Found: C, 75.33; H, 5.72; N, 5.45; F, 7.46.

(E)-O-[3-(3-Chlorophenyl)prop-2-enyl]benzaldehyde oxime (3Ag). A colorless oil. IR $\left(\mathrm{CHCl}_{3}\right) 2925,1477,1447,1425 \mathrm{~cm}^{-1} .{ }^{1} \mathrm{H} \mathrm{NMR}\left(\mathrm{CDCl}_{3}\right) \delta 8.13(1 \mathrm{H}, \mathrm{s}), 7.63-7.56(2 \mathrm{H}, \mathrm{m})$, 7.43-7.33 (4H, m), 7.28-7.17 (3H, m), $6.59(1 \mathrm{H}, \mathrm{d}, J=15.9 \mathrm{~Hz}), 6.42(1 \mathrm{H}, \mathrm{dt}, J=15.9,5.5$ $\mathrm{Hz}), 4.82(2 \mathrm{H}, \mathrm{d}, J=5.5 \mathrm{~Hz}) .{ }^{13} \mathrm{C}$ NMR $\left(\mathrm{CDCl}_{3}\right) \delta 149.1,138.6,134.5,132.2,131.8,129.9$, 129.8, 128.7, 127.7, 127.1, 126.9, 126.5, 124.8, 74.5. $\left.\mathrm{MS}_{(\mathrm{EI}}^{+}\right) \mathrm{m} / \mathrm{z}: 271\left(\mathrm{M}^{+}, 3\right), 151(100)$. HRMS calcd for $\mathrm{C}_{16} \mathrm{H}_{14} \mathrm{ClNO}$ : 271.0764, Found: 271.0764. 
(E)-O-[3-(4-Chlorophenyl)prop-2-enyl]benzaldehyde oxime (3Ah). A colorless crystal. mp 68-71 ${ }^{\circ} \mathrm{C}$ (hexane). IR $\left(\mathrm{CHCl}_{3}\right) 2926,1491,1448,1405 \mathrm{~cm}^{-1} .{ }^{1} \mathrm{H} \mathrm{NMR}\left(\mathrm{CDCl}_{3}\right) \delta 8.14$ $(1 \mathrm{H}, \mathrm{s}), 7.23-7.57(2 \mathrm{H}, \mathrm{m}), 7.40-7.27(7 \mathrm{H}, \mathrm{m}), 6.64(1 \mathrm{H}, \mathrm{d}, J=15.9 \mathrm{~Hz}), 6.40(1 \mathrm{H}, \mathrm{dt}$, $J=15.9,6.2 \mathrm{~Hz}), 4.83(2 \mathrm{H}, \mathrm{d}, J=6.2 \mathrm{~Hz}) .{ }^{13} \mathrm{C} \mathrm{NMR}\left(\mathrm{CDCl}_{3}\right) \delta 149.1,135.1,133.4,132.2$,

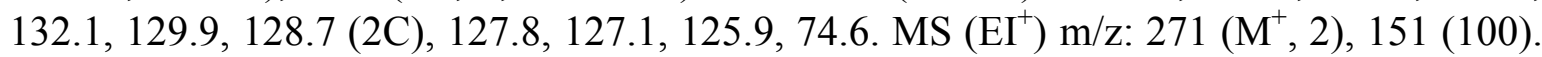
HRMS calcd for $\mathrm{C}_{16} \mathrm{H}_{14} \mathrm{ClNO}$ : 271.0764, Found: 271.0769. Anal. Calcd for $\mathrm{C}_{16} \mathrm{H}_{14} \mathrm{ClNO}$ : C, 70.72; H, 5.19; N, 5.15; Cl, 13.05. Found: C, 70.78; H, 5.32; N, 5.18; Cl, 13.25.

(E)-O-[3-(4-Methylphenyl)prop-2-enyl]benzaldehyde oxime (3Ai). A colorless crystal. mp $70-72{ }^{\circ} \mathrm{C}$ (hexane). IR $\left(\mathrm{CHCl}_{3}\right) 2925,1512,1493,1448,1414 \mathrm{~cm}^{-1} .{ }^{1} \mathrm{H} \mathrm{NMR}\left(\mathrm{CDCl}_{3}\right) \delta$ $8.14(1 \mathrm{H}, \mathrm{s}), 7.63-7.56(2 \mathrm{H}, \mathrm{m}), 7.40-7.34(3 \mathrm{H}, \mathrm{m}), 7.31(2 \mathrm{H}, \mathrm{d}, J=7.9 \mathrm{~Hz}), 7.12(2 \mathrm{H}, \mathrm{d}$, $J=7.9 \mathrm{~Hz}), 6.65(1 \mathrm{H}, \mathrm{d}, J=15.9 \mathrm{~Hz}), 6.38(1 \mathrm{H}, \mathrm{dt}, J=15.9,6.4 \mathrm{~Hz}), 4.83(2 \mathrm{H}, \mathrm{d}, J=6.4 \mathrm{~Hz})$, $2.34(3 \mathrm{H}, \mathrm{s}) .{ }^{13} \mathrm{C} \mathrm{NMR}\left(\mathrm{CDCl}_{3}\right) \delta 148.9,137.7,133.9,133.5,132.3,129.8,129.3,128.7$, 127.1, 126.5, 124.0, 75.0, 21.1. MS $\left(\mathrm{EI}^{+}\right) \mathrm{m} / \mathrm{z}: 251\left(\mathrm{M}^{+}, 1\right), 131$ (100). HRMS calcd for $\mathrm{C}_{17} \mathrm{H}_{17} \mathrm{NO}$ : 251.1310, Found: 251.1314. Anal. Calcd for $\mathrm{C}_{17} \mathrm{H}_{17} \mathrm{NO}$ : C, 81.24; $\mathrm{H}, 6.82$; $\mathrm{N}$, 5.57. Found: C, 81.54; H, 6.92; N, 5.53.

(E)-O-(3-Phenylprop-2-enyl)-4-trifluoromethylbenzaldehyde oxime (3Ba). A colorless crystal. mp 90-93 ${ }^{\circ} \mathrm{C}$ (hexane). IR $\left(\mathrm{CHCl}_{3}\right) 2928,1494,1450,1413 \mathrm{~cm}^{-1} .{ }^{1} \mathrm{H} \mathrm{NMR}\left(\mathrm{CDCl}_{3}\right)$ $\delta 8.15(1 \mathrm{H}, \mathrm{s}), 7.71(2 \mathrm{H}, \mathrm{d}, J=8.1 \mathrm{~Hz}), 7.60(2 \mathrm{H}, \mathrm{d}, J=8.1 \mathrm{~Hz}), 7.42(2 \mathrm{H}, \mathrm{d}, J=7.6 \mathrm{~Hz}), 7.36-$ $7.23(3 \mathrm{H}, \mathrm{m}), 6.70(1 \mathrm{H}, \mathrm{d}, J=15.9 \mathrm{~Hz}), 6.42(1 \mathrm{H}, \mathrm{dt}, J=15.9,6.4 \mathrm{~Hz}), 4.86(2 \mathrm{H}, \mathrm{d}, J=6.4$ $\mathrm{Hz}) .{ }^{13} \mathrm{C} \mathrm{NMR}\left(\mathrm{CDCl}_{3}\right) \delta 147.4,136.5,135.7,133.8,131.4(J=32.1 \mathrm{~Hz}), 128.6,128.0$,

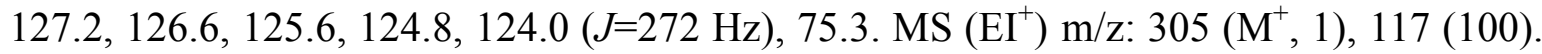
HRMS calcd for $\mathrm{C}_{17} \mathrm{H}_{14} \mathrm{~F}_{3} \mathrm{NO}$ : 305.1027, Found: 305.1023. Anal. Calcd for $\mathrm{C}_{17} \mathrm{H}_{14} \mathrm{~F}_{3} \mathrm{NO}$ : C, 66.88; H, 4.62; N, 4.59; F, 18.67. Found: C, 67.16; H, 4.81; N, 4.59; F, 18.67.

(E)-O-(3-Phenylprop-2-enyl)-4-methoxybenzaldehyde oxime (3Ca). A colorless crystal. mp $72-75{ }^{\circ} \mathrm{C}$ (hexane). IR $\left(\mathrm{CHCl}_{3}\right) 2935,1513,1463,1419 \mathrm{~cm}^{-1} .{ }^{1} \mathrm{H} \mathrm{NMR}\left(\mathrm{CDCl}_{3}\right) \delta 8.09$ $(1 \mathrm{H}, \mathrm{s}), 7.53(2 \mathrm{H}, \mathrm{d}, J=8.6 \mathrm{~Hz}), 7.40(2 \mathrm{H}, \mathrm{d}, J=7.5 \mathrm{~Hz}), 7.31(2 \mathrm{H}, \mathrm{d}, J=7.5 \mathrm{~Hz}), 7.23(1 \mathrm{H}$, $\mathrm{m}), 6.88(2 \mathrm{H}, \mathrm{d}, J=8.6 \mathrm{~Hz}), 6.69(1 \mathrm{H}, \mathrm{d}, J=15.9 \mathrm{~Hz}), 6.43(1 \mathrm{H}, \mathrm{dt}, J=15.9,6.2 \mathrm{~Hz}), 4.81$ $(2 \mathrm{H}, \mathrm{d}, J=6.2 \mathrm{~Hz}), 3.82(3 \mathrm{H}, \mathrm{s}) .{ }^{13} \mathrm{C} \mathrm{NMR}\left(\mathrm{CDCl}_{3}\right) \delta 161.0,148.6,136.7,133.3,128.5$

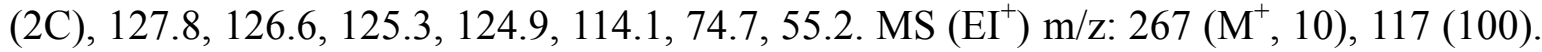
HRMS calcd for $\mathrm{C}_{17} \mathrm{H}_{17} \mathrm{NO}_{2}$ : 267.1259, Found: 267.1260. Anal. Calcd for $\mathrm{C}_{17} \mathrm{H}_{17} \mathrm{NO}_{2}$ : C, 76.38; H, 6.41; N, 5.24. Found: C, 76.52; H, 6.39; N, 5.22.

(E)-O-(3-Phenylprop-2-enyl)-2-pyridinecarboxaldehyde oxime (3Da). A colorless oil. IR $\left(\mathrm{CHCl}_{3}\right) 2928,1494,1470,1436 \mathrm{~cm}^{-1} .{ }^{1} \mathrm{H}$ NMR $\left(\mathrm{CDCl}_{3}\right) \delta 8.61(1 \mathrm{H}, \mathrm{d}, J=4.0 \mathrm{~Hz}), 8.23$ $(1 \mathrm{H}, \mathrm{s}), 7.80(1 \mathrm{H}, \mathrm{d}, J=7.9 \mathrm{~Hz}), 7.67(1 \mathrm{H}, \mathrm{m}), 7.41(2 \mathrm{H}, \mathrm{d}, J=7.6 \mathrm{~Hz}), 7.32(2 \mathrm{H}, \mathrm{d}, J=7.6$ $\mathrm{Hz}), 7.27-7.20(2 \mathrm{H}, \mathrm{m}), 6.69(1 \mathrm{H}, \mathrm{d}, J=15.9 \mathrm{~Hz}), 6.42(1 \mathrm{H}, \mathrm{dt}, J=15.9,6.4 \mathrm{~Hz}), 4.89(2 \mathrm{H}, \mathrm{d}$, $J=6.4 \mathrm{~Hz}) .{ }^{13} \mathrm{C} \mathrm{NMR}\left(\mathrm{CDCl}_{3}\right) \delta 151.7,149.7,149.6,136.5,136.4,133.7,128.6,127.9$, 126.6, 124.8, 124.0, 121.1, 75.4. MS (EI $\left.{ }^{+}\right) \mathrm{m} / \mathrm{z}: 238\left(\mathrm{M}^{+}, 2\right), 117$ (100). HRMS calcd for $\mathrm{C}_{15} \mathrm{H}_{14} \mathrm{~N}_{2} \mathrm{O}: 238.1106$, Found: 238.1114 . 
$\boldsymbol{O}$-(3-Phenylprop-2-enyl)benzophenone oxime (3Ea). ${ }^{2)} \mathrm{A}$ colorless crystal. mp $66-68{ }^{\circ} \mathrm{C}$ (hexane). IR $\left(\mathrm{CHCl}_{3}\right) 2924,1494,1444 \mathrm{~cm}^{-1} .{ }^{1} \mathrm{H} \mathrm{NMR}\left(\mathrm{CDCl}_{3}\right) \delta$ 7.55-7.20 (15H, m), 6.61 $(1 \mathrm{H}, \mathrm{d}, J=16.1 \mathrm{~Hz}), 6.41(1 \mathrm{H}, \mathrm{dt}, J=16.1,6.1 \mathrm{~Hz}), 4.86(2 \mathrm{H}, \mathrm{d}, J=6.1 \mathrm{~Hz}) .{ }^{13} \mathrm{C} \mathrm{NMR}$ $\left(\mathrm{CDCl}_{3}\right) \delta 157.0,136.8,136.6,133.4,132.9,129.4,129.3,128.9,128.6,128.2,128.1$, 128.0, 127.7, 126.6, 125.8, 75.2. MS $\left(\mathrm{EI}^{+}\right) \mathrm{m} / \mathrm{z}: 313\left(\mathrm{M}^{+}, 4\right), 117(100)$. HRMS calcd for $\mathrm{C}_{22} \mathrm{H}_{19} \mathrm{NO}$ : 313.1467, Found: 313.1469. Anal. Calcd for $\mathrm{C}_{22} \mathrm{H}_{19} \mathrm{NO}: \mathrm{C}, 84.31 ; \mathrm{H}, 6.11 ; \mathrm{N}$, 4.47. Found: C, 84.44; H, 6.16; N, 4.43.

$\boldsymbol{O}$-(3-Phenylprop-2-enyl)cyclohexanone oxime (3Fa). ${ }^{3)}$ A colorless oil. IR $\left(\mathrm{CHCl}_{3}\right)$ 2938, 1495, $1449 \mathrm{~cm}^{-1} .{ }^{1} \mathrm{H}$ NMR $\left(\mathrm{CDCl}_{3}\right) \delta 7.39(2 \mathrm{H}, \mathrm{d}, J=7.6 \mathrm{~Hz}), 7.30(2 \mathrm{H}, \mathrm{d}, J=7.6 \mathrm{~Hz}), 7.22$ $(1 \mathrm{H}$, br t, $J=7.6 \mathrm{~Hz}), 6.62(1 \mathrm{H}, \mathrm{d}, J=15.9 \mathrm{~Hz}), 6.37(1 \mathrm{H}, \mathrm{dt}, J=15.9,6.0 \mathrm{~Hz}), 4.68(2 \mathrm{H}, \mathrm{d}$, $J=6.0 \mathrm{~Hz}), 2.50(2 \mathrm{H}$, br t, $J=6.1 \mathrm{~Hz}), 2.21(2 \mathrm{H}$, br t, $J=6.1 \mathrm{~Hz}), 1.73-1.52(6 \mathrm{H}, \mathrm{m}) .{ }^{13} \mathrm{C}$ NMR $\left(\mathrm{CDCl}_{3}\right) \delta 160.5,136.9,132.5,128.5,127.6,126.5,125.9,73.8,32.1,26.9,25.7$, 25.6, 25.3. MS $\left(\mathrm{EI}^{+}\right) \mathrm{m} / \mathrm{z}: 229\left(\mathrm{M}^{+}, 2\right), 117$ (100). HRMS calcd for $\mathrm{C}_{15} \mathrm{H}_{19} \mathrm{NO}: 229.1467$, Found: 229.1467.

Methyl (E)-2-[(3-Phenylprop-2-enyloxy)imino]ethanate (3Ga). A colorless oil. IR $\left(\mathrm{CHCl}_{3}\right) 2954,1727,1494,1442 \mathrm{~cm}^{-1} .{ }^{1} \mathrm{H}$ NMR $\left(\mathrm{CDCl}_{3}\right) \delta 7.52(1 \mathrm{H}, \mathrm{s}), 7.40(2 \mathrm{H}, \mathrm{d}, J=7.3$ $\mathrm{Hz}), 7.32(2 \mathrm{H}, \mathrm{t}, J=7.3 \mathrm{~Hz}), 7.26(1 \mathrm{H}$, br t,$J=7.3 \mathrm{~Hz}), 6.67(1 \mathrm{H}, \mathrm{d}, J=15.9 \mathrm{~Hz}), 6.36(1 \mathrm{H}$, $\mathrm{dt}, J=15.9,6.6 \mathrm{~Hz}), 4.91(2 \mathrm{H}, \mathrm{d}, J=6.6 \mathrm{~Hz}), 3.86(3 \mathrm{H}, \mathrm{s}) .{ }^{13} \mathrm{C} \mathrm{NMR}\left(\mathrm{CDCl}_{3}\right) \delta 162.4,140.8$, 136.2, 134.7, 128.6, 128.1, 126.7, 123.4, 76.6, 52.4. MS (EI $\left.{ }^{+}\right) \mathrm{m} / \mathrm{z}: 219\left(\mathrm{M}^{+}, 6\right), 117(100)$. HRMS calcd for $\mathrm{C}_{12} \mathrm{H}_{13} \mathrm{NO}_{3}: 219.0895$, Found: 219.0897.

Benzylidene(3-phenylprop-2-enyl)amine $\boldsymbol{N}$-oxide (4Aa). Minor isomer ( $E$-isomer): A colorless solid. IR $\left(\mathrm{CHCl}_{3}\right)$ 2993, 1587, 1493, $1451 \mathrm{~cm}^{-1} .{ }^{1} \mathrm{H}$ NMR $\left(\mathrm{CDCl}_{3}\right) \delta$ 8.18-8.12 $(2 \mathrm{H}, \mathrm{m}), 7.39-7.19(9 \mathrm{H}, \mathrm{m}), 6.80(1 \mathrm{H}, \mathrm{d}, J=11.6 \mathrm{~Hz}), 6.04(1 \mathrm{H}, \mathrm{dt}, J=11.6,7.0 \mathrm{~Hz}), 4.75$ $(2 \mathrm{H}, \mathrm{d}, J=7.0 \mathrm{~Hz}) .{ }^{13} \mathrm{C} \mathrm{NMR}\left(\mathrm{CDCl}_{3}\right) \delta 135.7,134.9,134.1,130.5,130.4,128.7,128.6$, $128.5,127.9,123.4,65.0$. One peak of ${ }^{13} \mathrm{C}$ NMR was missing due to overlap. MS (EI ${ }^{+}$) $\mathrm{m} / \mathrm{z}$ : $237\left(\mathrm{M}^{+}, 5\right), 117$ (100). HRMS calcd for $\mathrm{C}_{16} \mathrm{H}_{15} \mathrm{NO}$ : 237.1154, Found: 237.1159. Major isomer $\left(Z\right.$-isomer): A colorless solid. IR $\left(\mathrm{CHCl}_{3}\right) 2989,1585,1494,1450 \mathrm{~cm}^{-1} .{ }^{1} \mathrm{H}$ NMR $\left(\mathrm{CDCl}_{3}\right) \delta 8.20-8.14(2 \mathrm{H}, \mathrm{m}), 7.38-7.16(9 \mathrm{H}, \mathrm{m}), 6.67(1 \mathrm{H}, \mathrm{d}, J=15.9 \mathrm{~Hz}), 6.45(1 \mathrm{H}$, $\mathrm{dt}, J=15.9,6.1 \mathrm{~Hz}), 4.62(2 \mathrm{H}, \mathrm{d}, J=6.1 \mathrm{~Hz}) .{ }^{13} \mathrm{C} \mathrm{NMR}\left(\mathrm{CDCl}_{3}\right) \delta 136.6,135.7,134.0,130.5$, 130.4, 128.7, 128.6, 128.5 (2C), 126.8, 121.0, 69.4. MS (EI $\left.{ }^{+}\right) \mathrm{m} / \mathrm{z}: 237\left(\mathrm{M}^{+}, 8\right), 117(100)$. HRMS calcd for $\mathrm{C}_{16} \mathrm{H}_{15} \mathrm{NO}$ : 237.1154, Found: 237.1164. 


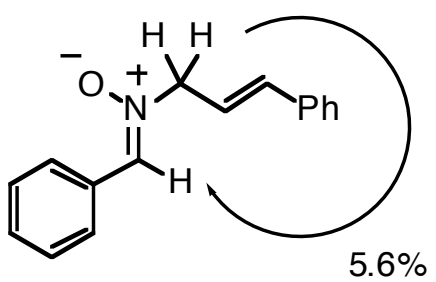

$Z-4 A a$

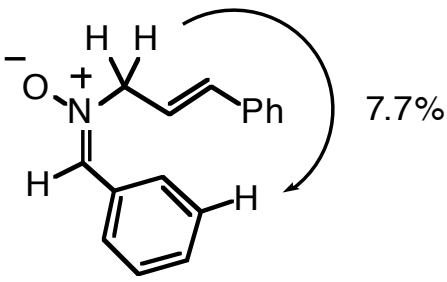

E-4Aa

FIGURE 1. The nOe studies of $Z$ - and $E$-nitrones $4 \mathrm{Aa}$

Benzylidene[3-(4-trifluoromethylphenyl)prop-2-enyl]amine $\mathbf{N}$-oxide (4Ab). Minor isomer: A colorless solid. IR $\left(\mathrm{CHCl}_{3}\right) 1582,1452 \mathrm{~cm}^{-1} .{ }^{1} \mathrm{H} \mathrm{NMR}\left(\mathrm{CDCl}_{3}\right) \delta 8.23(2 \mathrm{H}, \mathrm{m})$, $7.65(2 \mathrm{H}, \mathrm{d}, J=8.2 \mathrm{~Hz}), 7.47(2 \mathrm{H}, \mathrm{d}, J=8.2 \mathrm{~Hz}), 7.43(3 \mathrm{H}, \mathrm{m}), 7.38(1 \mathrm{H}, \mathrm{s}), 6.89(1 \mathrm{H}, \mathrm{d}$, $J=11.3 \mathrm{~Hz}), 6.21(1 \mathrm{H}, \mathrm{dt}, J=11.3,7.0 \mathrm{~Hz}), 4.79(2 \mathrm{H}, \mathrm{d}, J=7.0 \mathrm{~Hz}) .{ }^{13} \mathrm{C} \mathrm{NMR}\left(\mathrm{CDCl}_{3}\right) \delta$ 139.2, 134.4, 133.6, 130.7, 130.3, 129.9 (q, $J=33.1 \mathrm{~Hz}), 129.0,128.6$ (2C), 125.6 (q, $J=3.1$ $\mathrm{Hz}), 125.5,124.0$ (q, $J=272 \mathrm{~Hz})$, 64.7. $\mathrm{MS}\left(\mathrm{FAB}^{+}\right) \mathrm{m} / \mathrm{z}: 306\left(\mathrm{M}+\mathrm{H}^{+}, 51\right), 185$ (100). HRMS calcd for $\mathrm{C}_{17} \mathrm{H}_{15} \mathrm{NOF}_{3}\left(\mathrm{M}+\mathrm{H}^{+}\right)$: 306.1105, Found: 306.1101. Major isomer: A colorless solid. IR $\left(\mathrm{CHCl}_{3}\right) 1585,1452 \mathrm{~cm}^{-1} .{ }^{1} \mathrm{H} \mathrm{NMR}\left(\mathrm{CDCl}_{3}\right) \delta 8.24(2 \mathrm{H}, \mathrm{m}), 7.59(2 \mathrm{H}, \mathrm{d}, J=8.2$ $\mathrm{Hz}), 7.53(2 \mathrm{H}, \mathrm{d}, J=8.2 \mathrm{~Hz}), 7.49-7.41(4 \mathrm{H}, \mathrm{m}), 6.80(1 \mathrm{H}, \mathrm{d}, J=15.9 \mathrm{~Hz}), 6.65(1 \mathrm{H}, \mathrm{dt}$, $J=15.9,5.8 \mathrm{~Hz}), 4.74(2 \mathrm{H}, \mathrm{d}, J=5.8 \mathrm{~Hz}) .{ }^{13} \mathrm{C} \mathrm{NMR}\left(\mathrm{CDCl}_{3}\right) \delta 139.2,134.7,134.3,130.6$, $130.3,130.1$ (q, $J=32.1 \mathrm{~Hz}), 128.6,128.5,126.9,125.6$ (q, $J=3.1 \mathrm{~Hz}), 124.1$ (q, $J=272 \mathrm{~Hz})$, 124.0, 69.1. MS $\left(\mathrm{FAB}^{+}\right) \mathrm{m} / \mathrm{z}: 306\left(\mathrm{M}+\mathrm{H}^{+}, 55\right), 185$ (100). HRMS calcd for $\mathrm{C}_{17} \mathrm{H}_{15} \mathrm{NOF}_{3}$ $\left(\mathrm{M}+\mathrm{H}^{+}\right): 306.1105$, Found: 306.1107.

Benzylidene[3-(1-naphtyl)prop-2-enyl]amine $\mathbf{N}$-oxide (4Ad). Minor isomer: A colorless solid. IR $\left(\mathrm{CHCl}_{3}\right) 1588,1452 \mathrm{~cm}^{-1} .{ }^{1} \mathrm{H} \mathrm{NMR}\left(\mathrm{CDCl}_{3}\right) \delta 8.17(2 \mathrm{H}, \mathrm{m}), 7.96(1 \mathrm{H}, \mathrm{m}), 7.89$ $(1 \mathrm{H}, \mathrm{m}), 7.85(1 \mathrm{H}, \mathrm{d}, J=8.2 \mathrm{~Hz}), 7.56-7.38(7 \mathrm{H}, \mathrm{m}), 7.34(1 \mathrm{H}, \mathrm{d}, J=11.3 \mathrm{~Hz}), 7.22(1 \mathrm{H}, \mathrm{s})$, $6.43(1 \mathrm{H}, \mathrm{dt}, J=11.3,7.0 \mathrm{~Hz}), 4.66(2 \mathrm{H}, \mathrm{d}, J=7.0 \mathrm{~Hz}) .{ }^{13} \mathrm{C} \mathrm{NMR}\left(\mathrm{CDCl}_{3}\right) \delta 134.2,133.6$, 133.3, 132.8, 131.6, 130.5, 130.4, 128.6 (2C), 128.5 (2C), 126.5, 126.4, 126.2, 125.6, 125.4, 124.7, 65.2. MS $\left(\mathrm{FAB}^{+}\right) \mathrm{m} / \mathrm{z}: 288\left(\mathrm{M}+\mathrm{H}^{+}, 25\right), 167$ (100). HRMS calcd for $\mathrm{C}_{20} \mathrm{H}_{18} \mathrm{NO}\left(\mathrm{M}+\mathrm{H}^{+}\right)$: 288.1388, Found: 188.1393. Major isomer: A colorless solid. IR $\left(\mathrm{CHCl}_{3}\right) 1588,1451 \mathrm{~cm}^{-1} .{ }^{1} \mathrm{H}$ NMR $\left(\mathrm{CDCl}_{3}\right) \delta 8.26(2 \mathrm{H}, \mathrm{m}), 8.11(1 \mathrm{H}, \mathrm{d}, J=8.2 \mathrm{~Hz}), 7.85$ $(1 \mathrm{H}, \mathrm{d}, J=8.2 \mathrm{~Hz}), 7.81(1 \mathrm{H}, \mathrm{d}, J=8.2 \mathrm{~Hz}), 7.66(1 \mathrm{H}, \mathrm{d}, J=7.3 \mathrm{~Hz}), 7.56-7.39(8 \mathrm{H}, \mathrm{m}), 6.56$ $(1 \mathrm{H}, \mathrm{dt}, J=15.6,6.7 \mathrm{~Hz}), 4.82(2 \mathrm{H}, \mathrm{d}, J=6.7 \mathrm{~Hz}) .{ }^{13} \mathrm{C} \mathrm{NMR}\left(\mathrm{CDCl}_{3}\right) \delta 134.2,133.9,133.6$, $133.3,131.0,130.5,130.4,128.8,128.7,128.6,128.5,126.4,125.9,125.6,124.4,124.1$, 123.5, 69.5. MS $\left(\mathrm{FAB}^{+}\right) \mathrm{m} / \mathrm{z}: 288\left(\mathrm{M}+\mathrm{H}^{+}, 4.1\right), 167(100)$. HRMS calcd for $\mathrm{C}_{20} \mathrm{H}_{18} \mathrm{NO}$ $\left(\mathrm{M}+\mathrm{H}^{+}\right): 288.1388$, Found: 188.1382 .

Benzylidene[3-(4-chlorophenyl)prop-2-enyl]amine $\mathbf{N}$-oxide (4Ah). Minor isomer: A colorless solid. IR $\left(\mathrm{CHCl}_{3}\right)$ 1589, 1490, $1452 \mathrm{~cm}^{-1} .{ }^{1} \mathrm{H}$ NMR $\left(\mathrm{CDCl}_{3}\right) \delta 8.23(2 \mathrm{H}, \mathrm{m}), 7.46-$ $7.24(8 \mathrm{H}, \mathrm{m}), 6.81(1 \mathrm{H}, \mathrm{d}, J=11.6 \mathrm{~Hz}), 6.12(1 \mathrm{H}, \mathrm{dt}, J=11.6,7.0 \mathrm{~Hz}), 4.78(2 \mathrm{H}, \mathrm{d}, J=7.0$ $\mathrm{Hz}) .{ }^{13} \mathrm{C} \mathrm{NMR}\left(\mathrm{CDCl}_{3}\right) \delta 134.3,134.1,133.8,130.6,130.4,130.0,128.8,128.6(2 \mathrm{C})$, 
124.1, 64.8. One peak of ${ }^{13} \mathrm{C}$ NMR was missing due to overlap. MS $\left(\mathrm{FAB}^{+}\right) \mathrm{m} / \mathrm{z}: 272$ $\left(\mathrm{M}+\mathrm{H}^{+}\right.$, 55), 151 (100). HRMS calcd for $\mathrm{C}_{16} \mathrm{H}_{15} \mathrm{NOCl}\left(\mathrm{M}+\mathrm{H}^{+}\right)$: 272.0842, Found: 272.0841. Major isomer: A colorless solid. IR $\left(\mathrm{CHCl}_{3}\right)$ 1590. 1492, $1452 \mathrm{~cm}^{-1} .{ }^{1} \mathrm{H} \mathrm{NMR}$ $\left(\mathrm{CDCl}_{3}\right) \delta 8.23(2 \mathrm{H}, \mathrm{m}), 7.47-7.25(8 \mathrm{H}, \mathrm{m}), 6.70(1 \mathrm{H}, \mathrm{d}, J=15.9 \mathrm{~Hz}), 6.50(1 \mathrm{H}, \mathrm{dt}, J=15.9$, $7.0 \mathrm{~Hz}), 4.68(2 \mathrm{H}, \mathrm{d}, J=7.0 \mathrm{~Hz}) .{ }^{13} \mathrm{C} \mathrm{NMR}\left(\mathrm{CDCl}_{3}\right) \delta 135.1,134.2,134.1,130.5,130.3$, 128.8, 128.6, 128.5, 128.0, 121.8, 69.2. One peak of ${ }^{13} \mathrm{C}$ NMR was missing due to overlap. MS $\left(\mathrm{FAB}^{+}\right) \mathrm{m} / \mathrm{z}: 272\left(\mathrm{M}+\mathrm{H}^{+}, 29\right), 151$ (100). HRMS calcd for $\mathrm{C}_{16} \mathrm{H}_{15} \mathrm{NOCl}\left(\mathrm{M}+\mathrm{H}^{+}\right)$: 272.0842, Found: 272.0851.

Benzylidene[3-(4-methylphenyl)prop-2-enyl]amine $\mathbf{N}$-oxide (4Ai). Minor isomer: A colorless solid. IR $\left(\mathrm{CHCl}_{3}\right) 1596,1450 \mathrm{~cm}^{-1} .{ }^{1} \mathrm{H}$ NMR $\left(\mathrm{CDCl}_{3}\right) \delta 8.23(2 \mathrm{H}, \mathrm{m}), 7.48-7.10$ $(8 \mathrm{H}, \mathrm{m}), 6.83(1 \mathrm{H}, \mathrm{d}, J=11.6 \mathrm{~Hz}), 6.06(1 \mathrm{H}, \mathrm{dt}, J=11.6,7.0 \mathrm{~Hz}), 4.82(2 \mathrm{H}, \mathrm{d}, J=7.0 \mathrm{~Hz})$, $2.37(3 \mathrm{H}, \mathrm{s}) .{ }^{13} \mathrm{C} \mathrm{NMR}\left(\mathrm{CDCl}_{3}\right) \delta 137.8,134.8,134.0,132.8,131.7,130.5,129.3,128.6$, $128.5,122.6,65.1,21.2$. One peak of ${ }^{13} \mathrm{C}$ NMR was missing due to overlap. MS (FAB ${ }^{+}$) $\mathrm{m} / \mathrm{z}: 252\left(\mathrm{M}+\mathrm{H}^{+}, 11\right), 131$ (100). HRMS calcd for $\mathrm{C}_{17} \mathrm{H}_{18} \mathrm{NO}\left(\mathrm{M}+\mathrm{H}^{+}\right): 252.1389$, Found: 252.1393. Major isomer: A colorless solid. IR $\left(\mathrm{CHCl}_{3}\right) 1586,1513,1451 \mathrm{~cm}^{-1}$. ${ }^{1} \mathrm{H}$ NMR $\left(\mathrm{CDCl}_{3}\right) \delta 8.23(2 \mathrm{H}, \mathrm{m}), 7.45(1 \mathrm{H}, \mathrm{s}), 7.41(3 \mathrm{H}, \mathrm{m}), 7.33(2 \mathrm{H}, \mathrm{d}, J=7.9 \mathrm{~Hz}), 7.15(2 \mathrm{H}, \mathrm{d}$, $J=7.9 \mathrm{~Hz}), 6.73(1 \mathrm{H}, \mathrm{d}, J=15.9 \mathrm{~Hz}), 6.46(1 \mathrm{H}, \mathrm{dt}, J=15.9,7.0 \mathrm{~Hz}), 4.69(2 \mathrm{H}, \mathrm{d}, J=7.0 \mathrm{~Hz})$, $2.34(3 \mathrm{H}, \mathrm{s}) .{ }^{13} \mathrm{C} \mathrm{NMR}\left(\mathrm{CDCl}_{3}\right) \delta 138.5,136.7,133.9,133.0,130.5(2 \mathrm{C}), 129.4,128.6$, 128.5, 126.7, 119.8, 69.5, 21.2. MS $\left(\mathrm{FAB}^{+}\right) \mathrm{m} / \mathrm{z}: 252\left(\mathrm{M}+\mathrm{H}^{+}, 9\right), 131$ (100). HRMS calcd for $\mathrm{C}_{17} \mathrm{H}_{18} \mathrm{NO}\left(\mathrm{M}+\mathrm{H}^{+}\right): 252.1389$, Found: 252.1386 .

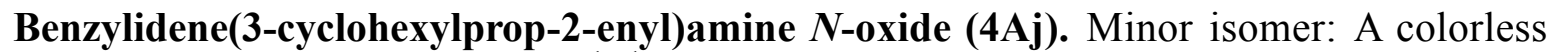
solid. IR $\left(\mathrm{CHCl}_{3}\right)$ 1596, $1450 \mathrm{~cm}^{-1} .{ }^{1} \mathrm{H} \mathrm{NMR}\left(\mathrm{CDCl}_{3}\right) \delta 8.23(2 \mathrm{H}, \mathrm{m}), 7.47-7.39(3 \mathrm{H}, \mathrm{m})$, 5.75-7.60 (2H, m), $4.61(2 \mathrm{H}, \mathrm{d}, J=6.7 \mathrm{~Hz}), 2.35(1 \mathrm{H}, \mathrm{m}), 1.82-1.45(5 \mathrm{H}, \mathrm{m}), 1.37-1.18(5 \mathrm{H}$, m). MS $\left(\mathrm{FAB}^{+}\right) \mathrm{m} / \mathrm{z}: 244\left(\mathrm{M}+\mathrm{H}^{+}, 67\right), 81(100)$. HRMS calcd for $\mathrm{C}_{16} \mathrm{H}_{22} \mathrm{NO}\left(\mathrm{M}+\mathrm{H}^{+}\right)$: 244.1701, Found: 244.1700. Major isomer: A colorless solid. IR $\left(\mathrm{CHCl}_{3}\right) 2853,1582,1450$ $\mathrm{cm}^{-1} .{ }^{1} \mathrm{H}$ NMR $\left(\mathrm{CDCl}_{3}\right) \delta 8.23(2 \mathrm{H}, \mathrm{m}), 7.45-7.35(4 \mathrm{H}, \mathrm{m}), 5.84(1 \mathrm{H}, \mathrm{dd}, J=15.6,6.4 \mathrm{~Hz})$, $5.76(1 \mathrm{H}, \mathrm{dt}, J=15.6,6.7 \mathrm{~Hz}), 4.48(2 \mathrm{H}, \mathrm{d}, J=6.7 \mathrm{~Hz}), 2.05(1 \mathrm{H}, \mathrm{m}), 1.80-1.62(5 \mathrm{H}, \mathrm{m})$, 1.35-1.05 (5H, m). ${ }^{13} \mathrm{C} \mathrm{NMR}\left(\mathrm{CDCl}_{3}\right) \delta 144.8,133.5,130.5,130.3,128.6,128.5,119.6$, 69.5, 40.4, 32.4, 26.0, 25.8. MS (FAB $\left.{ }^{+}\right) \mathrm{m} / \mathrm{z}: 244\left(\mathrm{M}+\mathrm{H}^{+}, 100\right)$. HRMS calcd for $\mathrm{C}_{16} \mathrm{H}_{22} \mathrm{NO}$ $\left(\mathrm{M}+\mathrm{H}^{+}\right): 244.1701$, Found: 244.1710 .

Diphenylmethylidene(3-phenylprop-2-enyl)amine $\mathbf{N}$-oxide (4Ea). A colorless solid. IR $\left(\mathrm{CHCl}_{3}\right) 1655,1438 \mathrm{~cm}^{-1} .{ }^{1} \mathrm{H}$ NMR $\left(\mathrm{CDCl}_{3}\right) \delta 8.03(2 \mathrm{H}, \mathrm{m}), 7.53-7.47(3 \mathrm{H}, \mathrm{m}), 7.39-7.24$ $(10 \mathrm{H}, \mathrm{m}), 6.52(1 \mathrm{H}, \mathrm{dt}, J=15.6,6.4 \mathrm{~Hz}), 6.40(1 \mathrm{H}, \mathrm{d}, J=15.6 \mathrm{~Hz}), 4.59(1 \mathrm{H}, \mathrm{d}, J=6.4 \mathrm{~Hz})$. ${ }^{13} \mathrm{C} \mathrm{NMR}\left(\mathrm{CDCl}_{3}\right) \delta 136.1,135.1,133.6,130.3,130.1,130.0,129.8,129.7,129.1,128.6$, 128.3, 128.2, 127.9, 126.7, 122.0, 66.1. MS (EI $\left.{ }^{+}\right) \mathrm{m} / \mathrm{z}: 313\left(\mathrm{M}^{+}, 1.9\right), 117$ (100). HRMS calcd for $\mathrm{C}_{22} \mathrm{H}_{19} \mathrm{NO}\left(\mathrm{M}^{+}\right)$: 313.1467, Found: 313.1476 .

(E)-O-(1-Phenylprop-2-enyl)benzaldehyde oxime (5Aa). A colorless oil. IR $\left(\mathrm{CHCl}_{3}\right)$ 2908, 1494, 1450, $1414 \mathrm{~cm}^{-1} .{ }^{1} \mathrm{H}$ NMR $\left(\mathrm{CDCl}_{3}\right) \delta 8.18(1 \mathrm{H}, \mathrm{s}), 7.60-7.50(2 \mathrm{H}, \mathrm{m}), 7.45-$ $7.23(8 \mathrm{H}, \mathrm{m}), 6.16(1 \mathrm{H}, \mathrm{ddd}, J=17.1,10.5,6.3 \mathrm{~Hz}), 5.71(1 \mathrm{H}, \mathrm{d}, J=6.3 \mathrm{~Hz}), 5.34(1 \mathrm{H}, \mathrm{d}$, 
$J=17.1 \mathrm{~Hz}), 5.29(1 \mathrm{H}, \mathrm{d}, J=10.5 \mathrm{~Hz}) \cdot{ }^{13} \mathrm{C} \mathrm{NMR}\left(\mathrm{CDCl}_{3}\right) \delta 149.2,140.1,137.6,132.3$, 129.8, 128.6, 128.4, 127.9, 127.4, 127.1, 117.3. MS (EI $\left.{ }^{+}\right) \mathrm{m} / \mathrm{z}: 237\left(\mathrm{M}^{+}, 0.2\right), 117$ (100). HRMS calcd for $\mathrm{C}_{16} \mathrm{H}_{15} \mathrm{NO}$ : 237.1154, Found: 237.1156.

Benzylidene(1,3-diphenylprop-2-enyl)amine $\boldsymbol{N}$-oxide (8). A colorless solid. IR $\left(\mathrm{CHCl}_{3}\right)$ 1581, 1494, $1451 \mathrm{~cm}^{-1} .{ }^{1} \mathrm{H}$ NMR $\left(\mathrm{CDCl}_{3}\right) \delta 8.25(2 \mathrm{H}, \mathrm{m}), 7.60-7.25(14 \mathrm{H}, \mathrm{m}), 6.86(1 \mathrm{H}$, $\mathrm{dd}, J=16.2,7.9 \mathrm{~Hz}), 6.69(1 \mathrm{H}, \mathrm{d}, J=16.2 \mathrm{~Hz}), 5.75(1 \mathrm{H}, \mathrm{d}, J=7.9 \mathrm{~Hz}) .{ }^{13} \mathrm{C}$ NMR $\left(\mathrm{CDCl}_{3}\right) \delta$ 137.2, 135.9, 135.2, 133.5, 130.5, 130.4, 128.9, 128.8, 128.7, 128.5 (2C), 127.8, 126.9, 125.2, 82.0. One peak of ${ }^{13} \mathrm{C}$ NMR was missing due to overlap. $\mathrm{MS}\left(\mathrm{CI}^{+}\right) \mathrm{m} / \mathrm{z}: 314\left(\mathrm{M}^{+} \mathrm{H}^{+}\right.$, 0.6), 193 (100). HRMS calcd for $\mathrm{C}_{22} \mathrm{H}_{20} \mathrm{NO}\left(\mathrm{M}+\mathrm{H}^{+}\right)$: 314.1545 , Found: 314.1543.

\section{References}

1) (a) Davies, S. G.; Jones, S.; Sanz, M. A.; Teixeira, F. C.; Fox, J. F. Chem. Commun. 1998, 2235. (b) Tiecco, M.; Testaferri, L.; Tingoli, M.; Bagnoli, L. Chem. Commun. $1995,235$.

2) (a) Tiecco, M.; Testaferri, L.; Marini, F.; Sternativo, S.; Santi, C.; Bagnoli, L.; Temperini, A. Tetrahedron: Asymmetry. 2001, 12, 3059. (b) Tiecco, M.; Testaferri, L.; Tingoli, M.; Bagnoli, L. Chem. Commun. 1995, 235.

3) (a) Koyama, J.; Ogura, T.; Tagahara, K.; Miyashita, M.; Irie, H. Chem. Pharm. Bull. 1993, 41, 1297. (b) Koyama, J.; Ogura, T.; Tagahara, K.; Irie, H. Chemistry Express 1991, 6, 197.

\section{Copies of ${ }^{1} \mathrm{H}$ and ${ }^{13} \mathrm{C}$ NMR spectra of all obtained compounds}



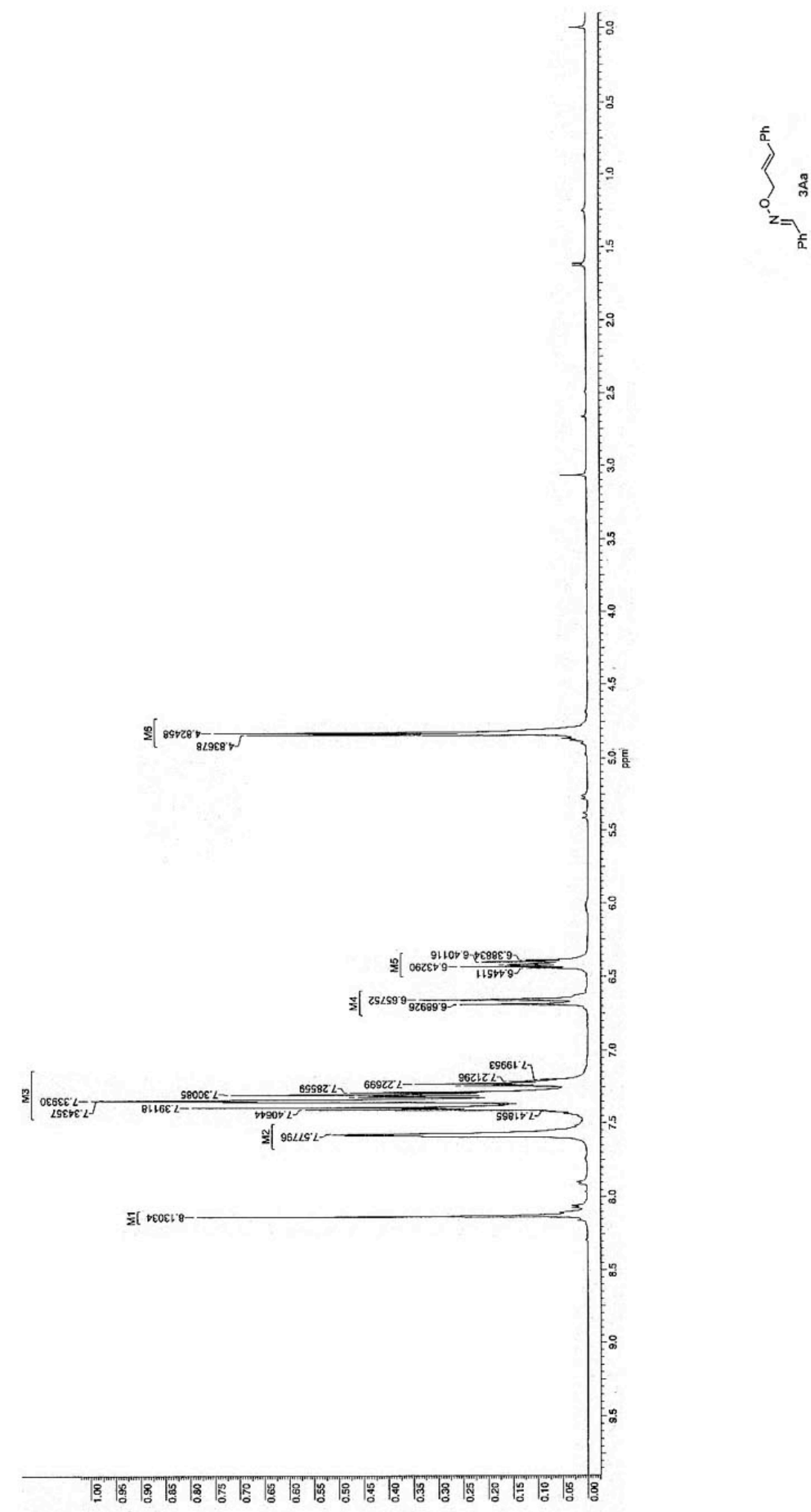


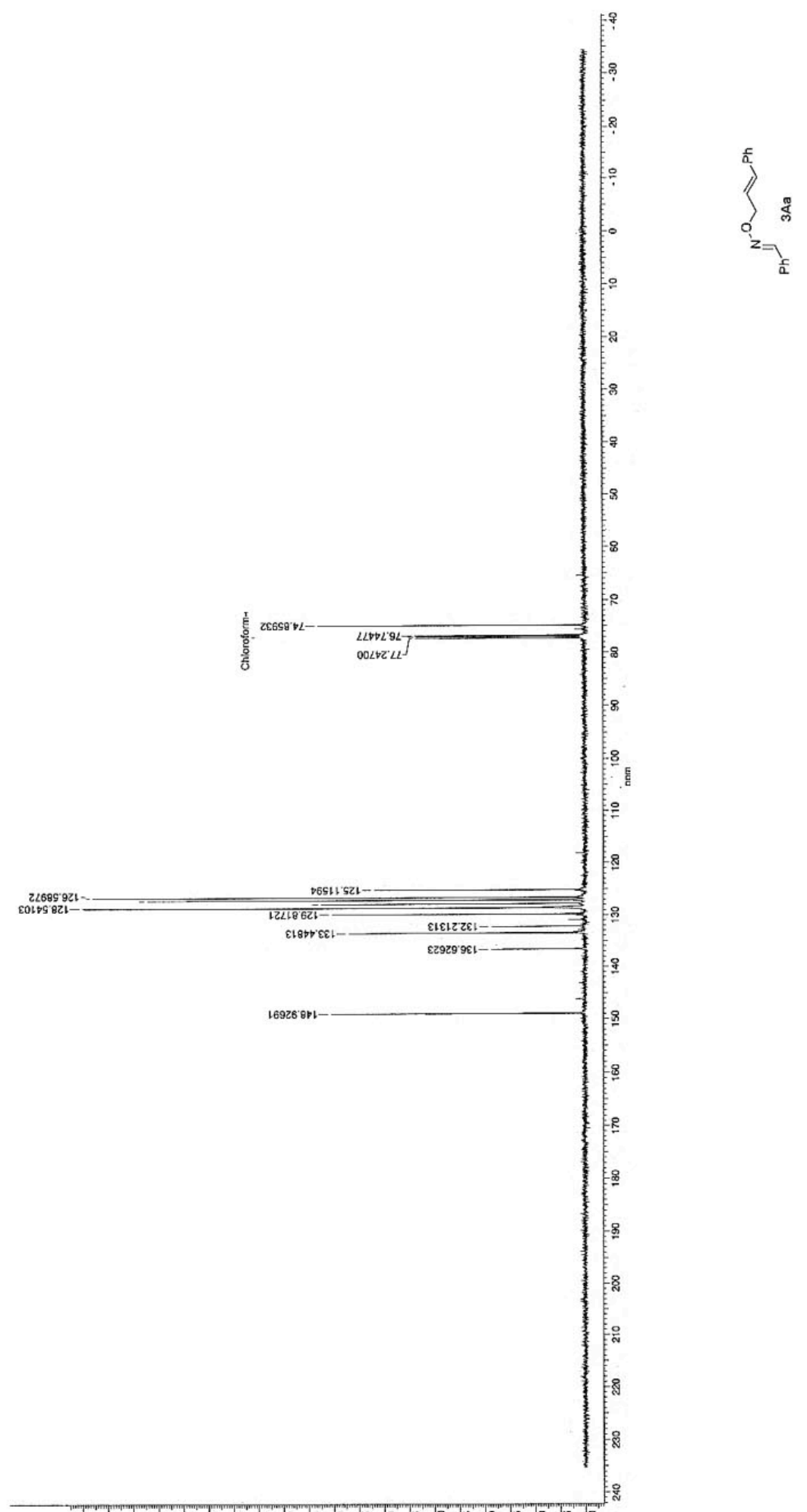

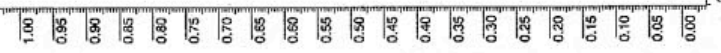




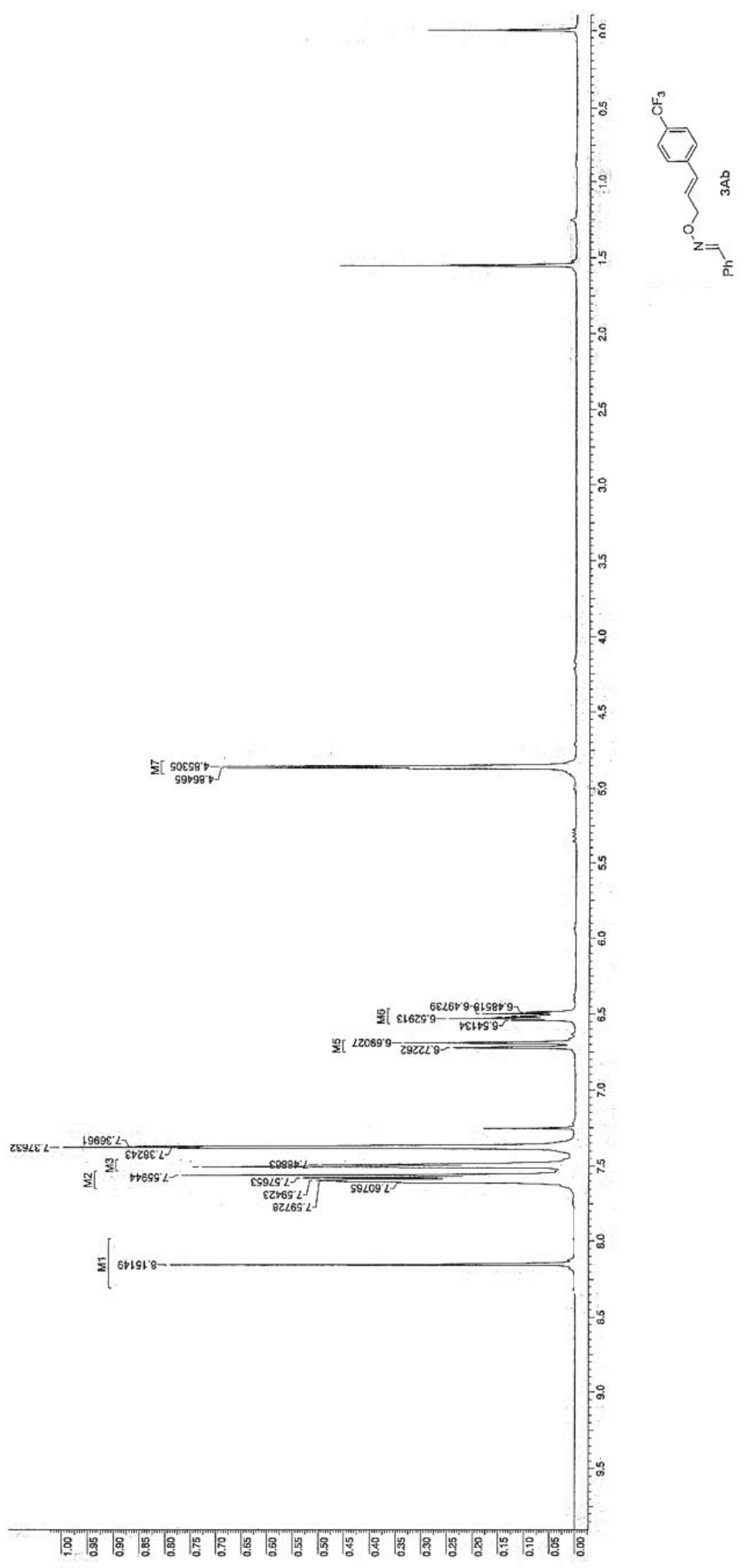




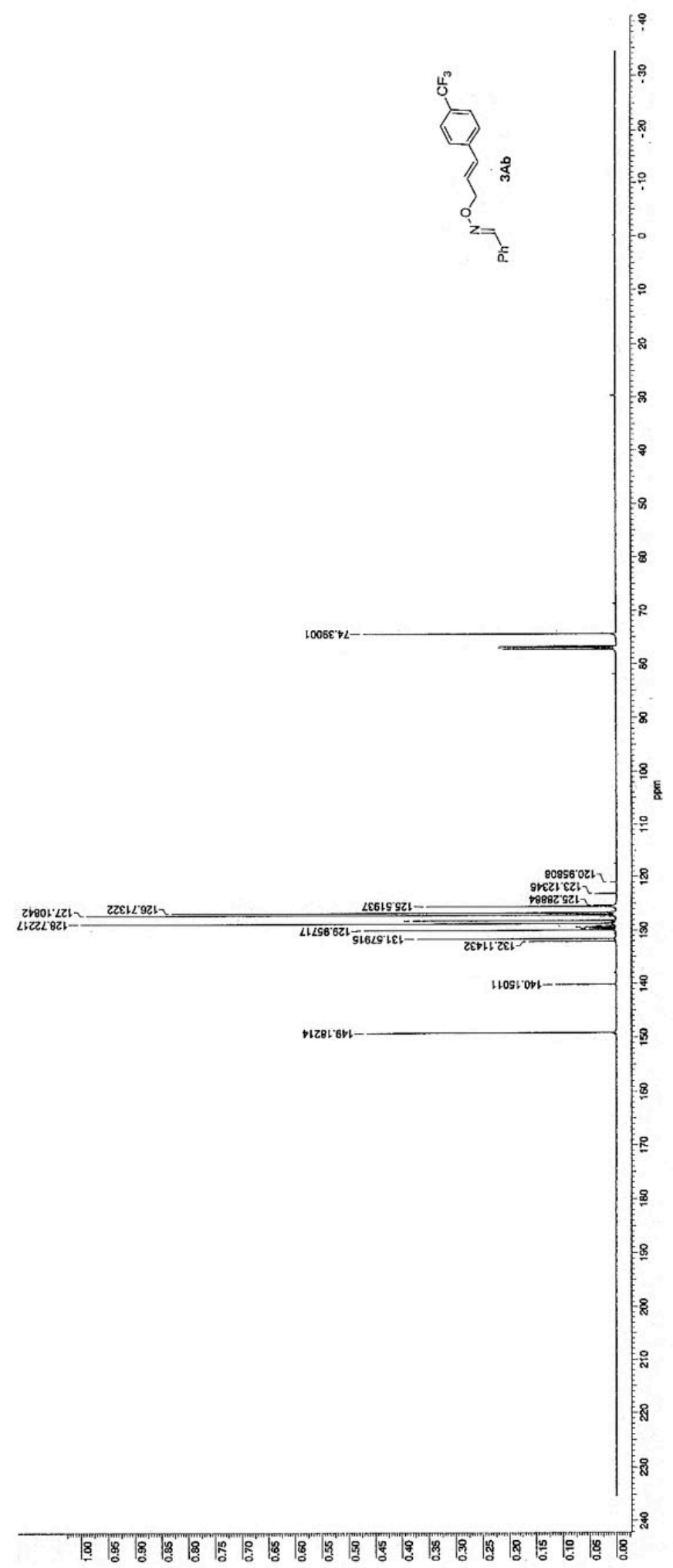




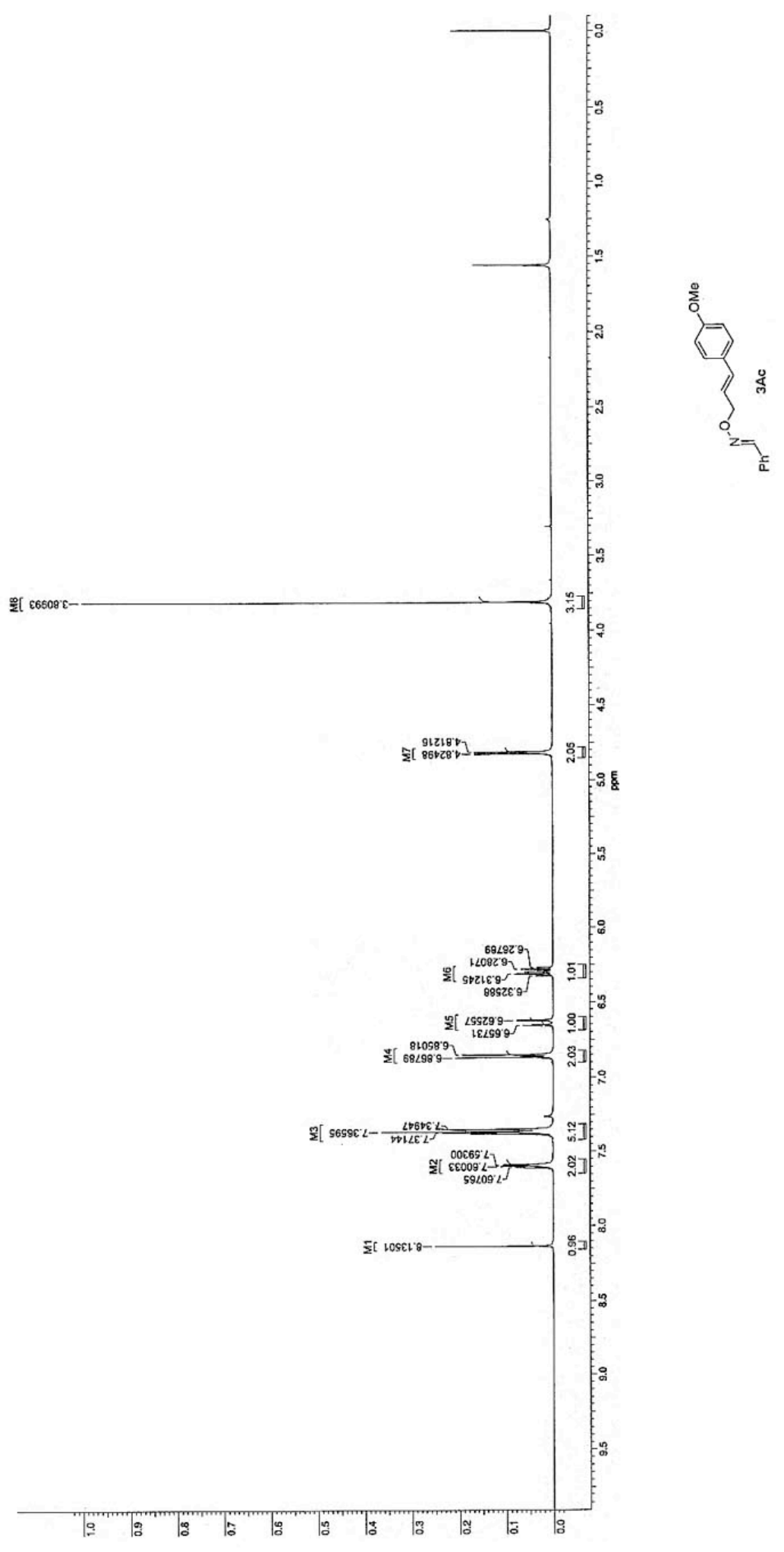




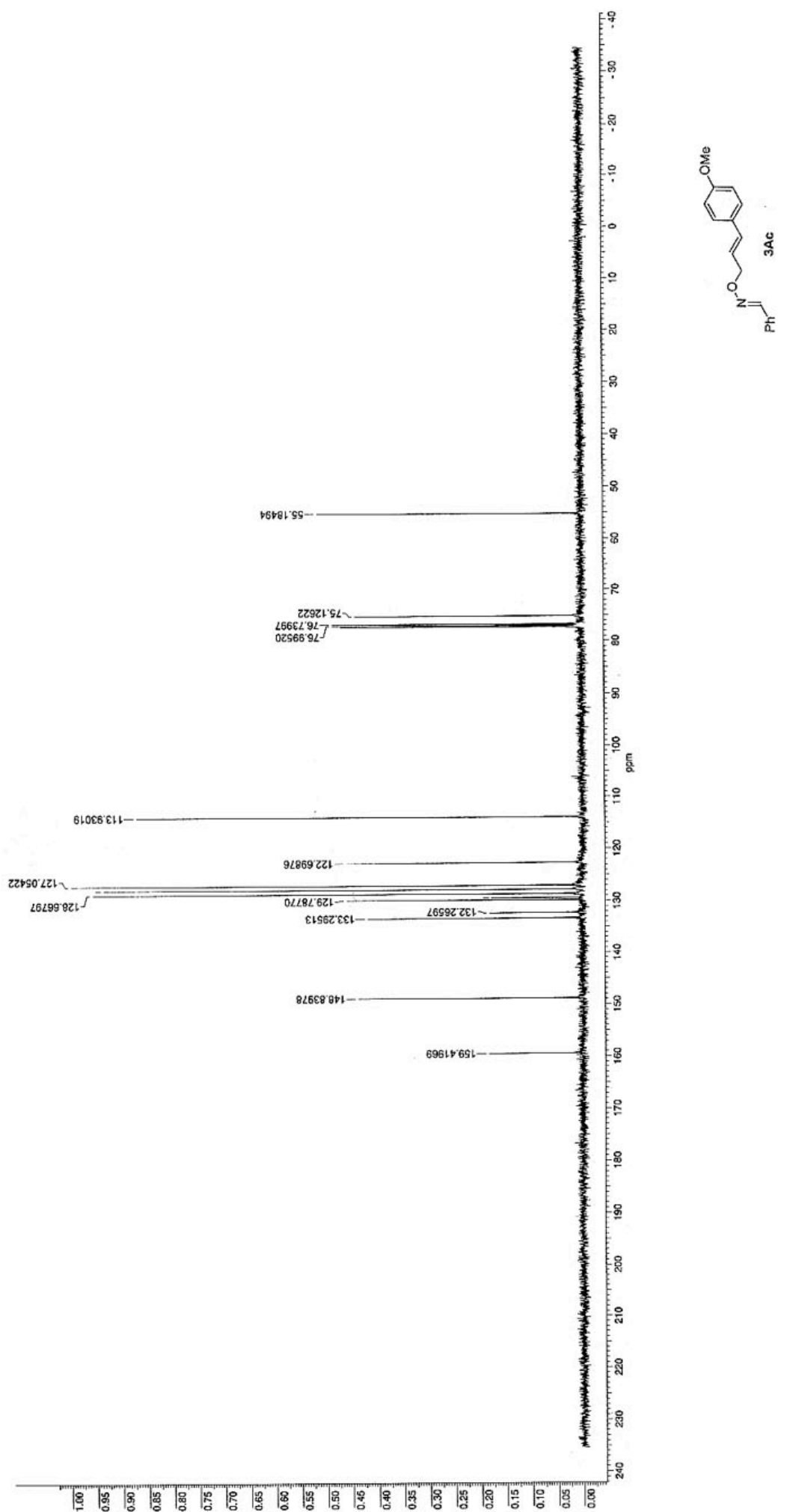




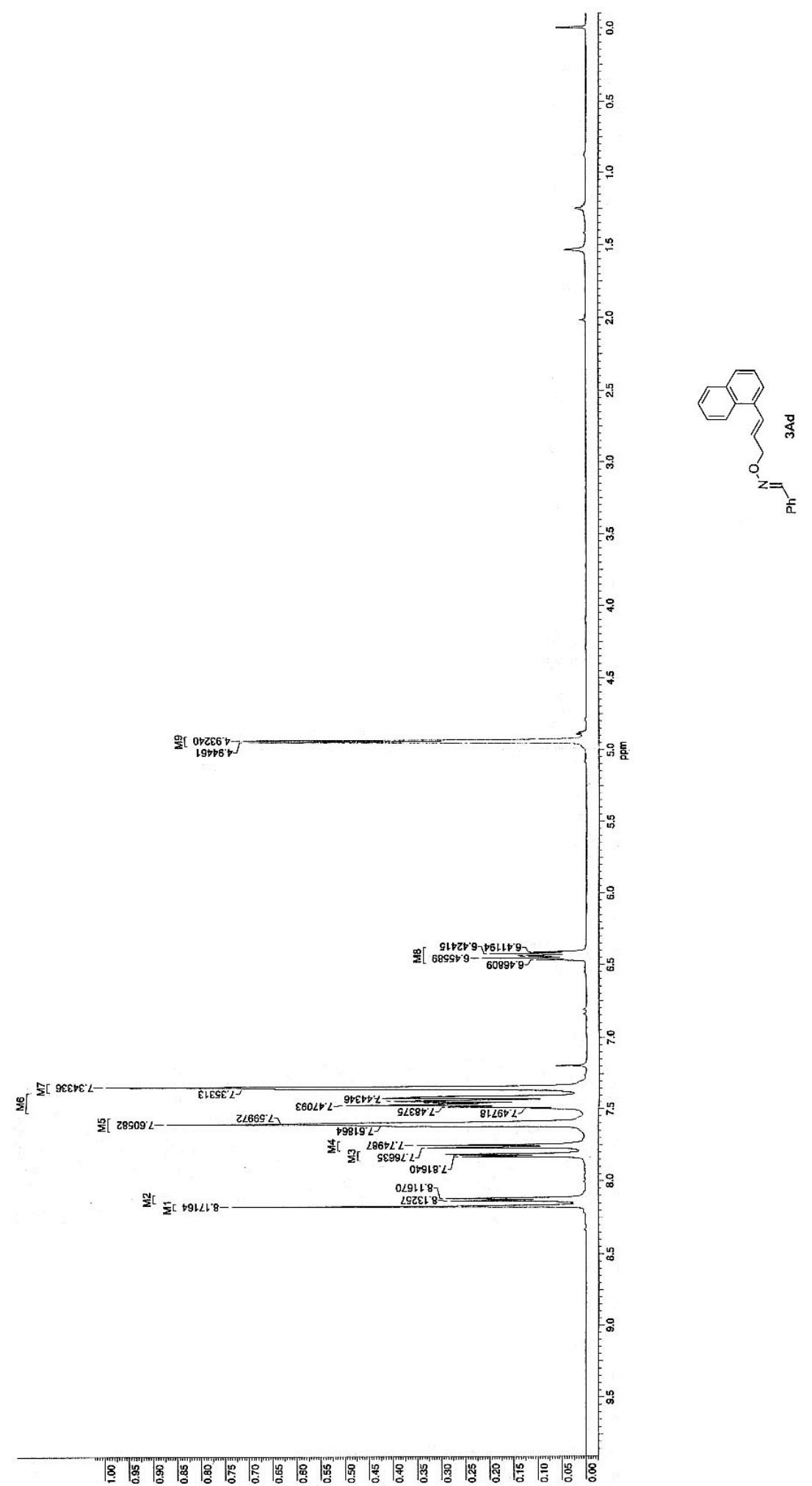




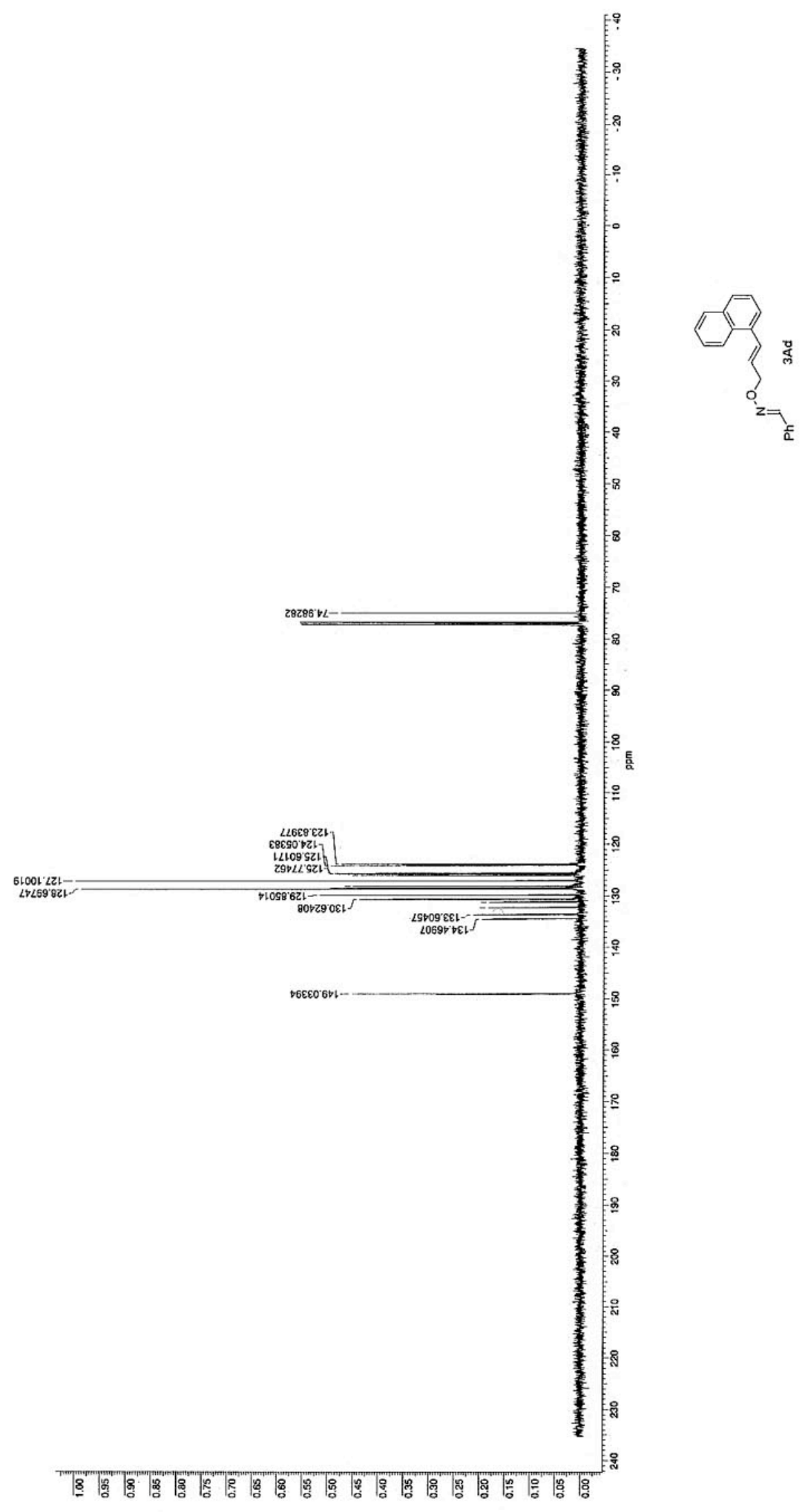




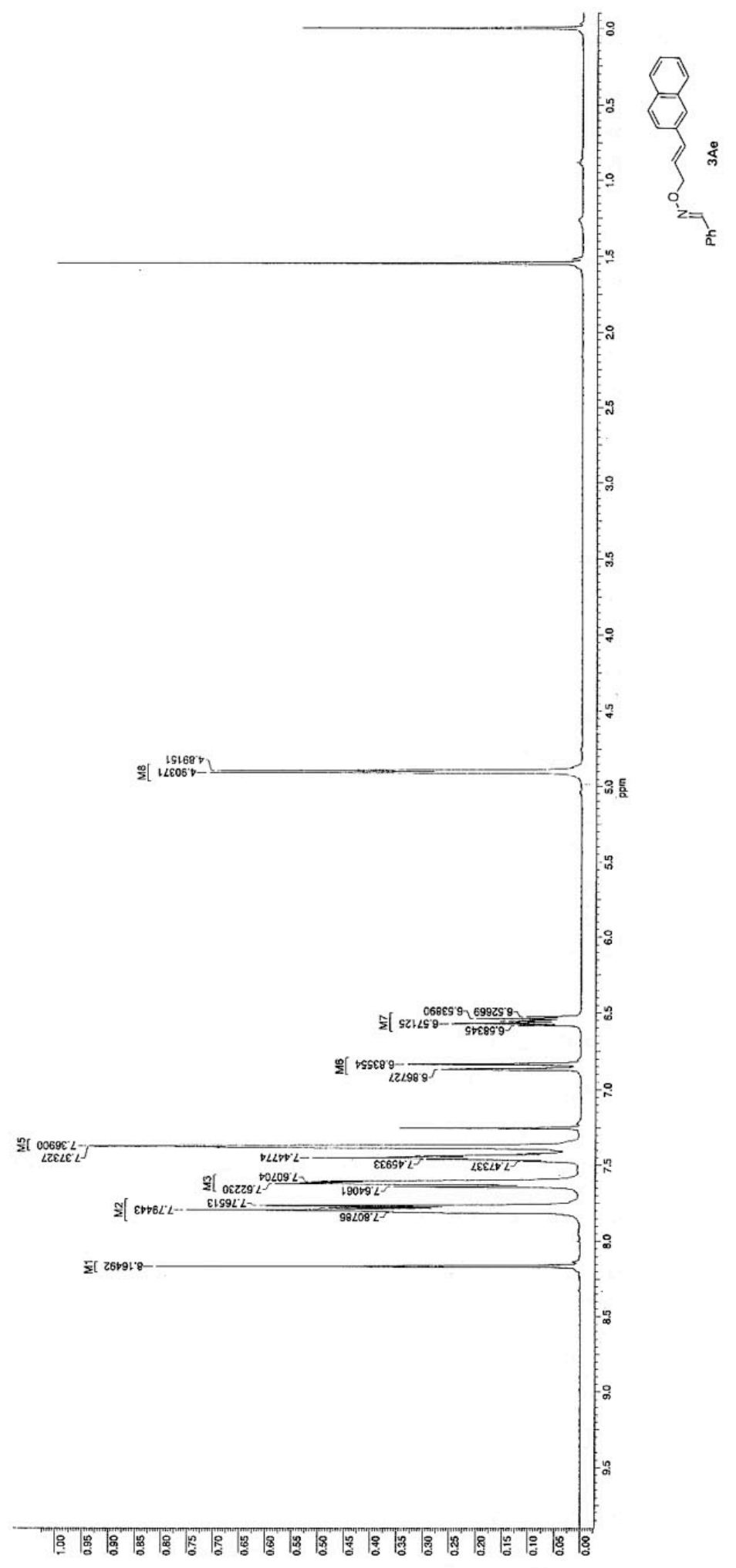




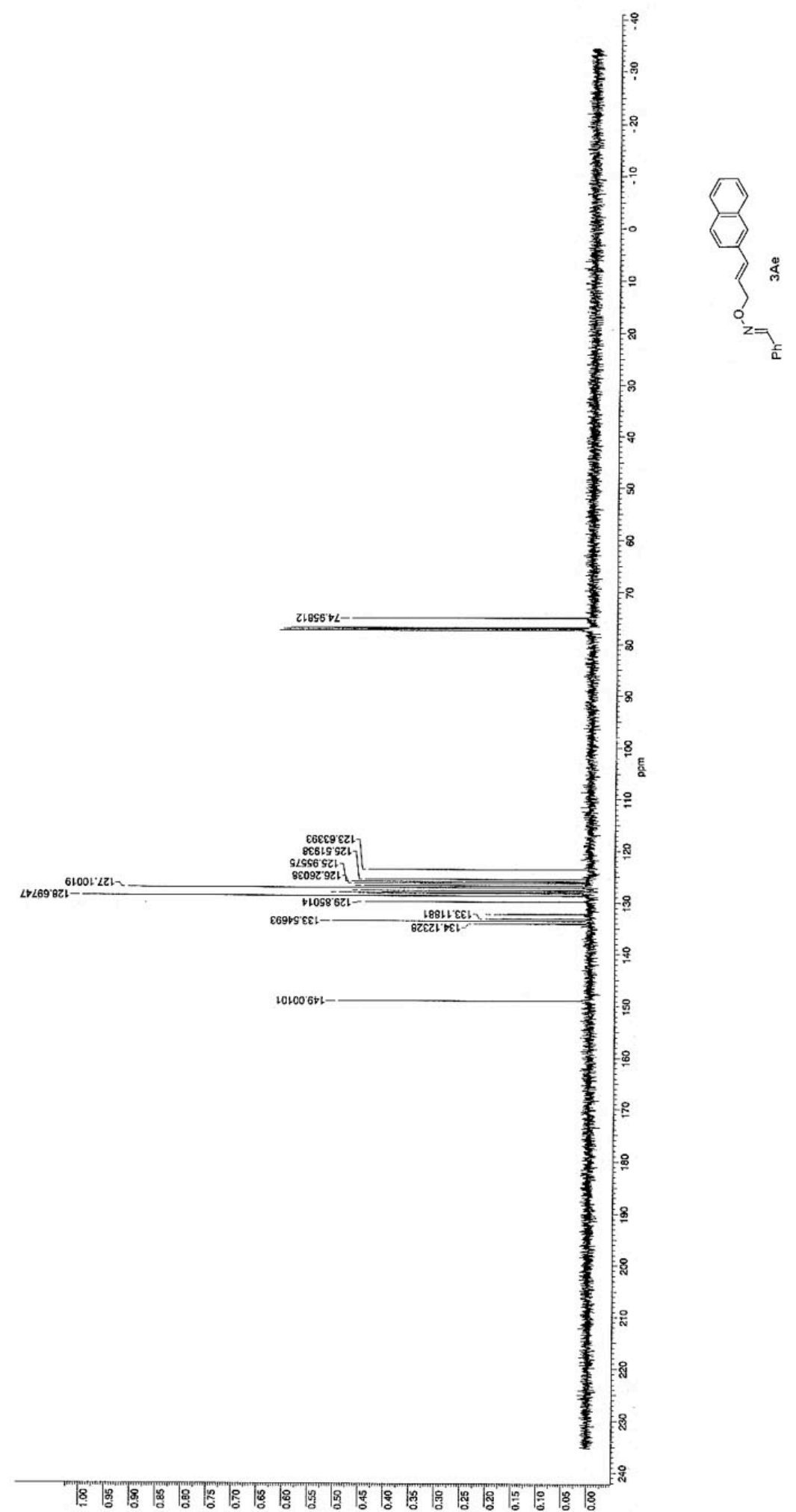




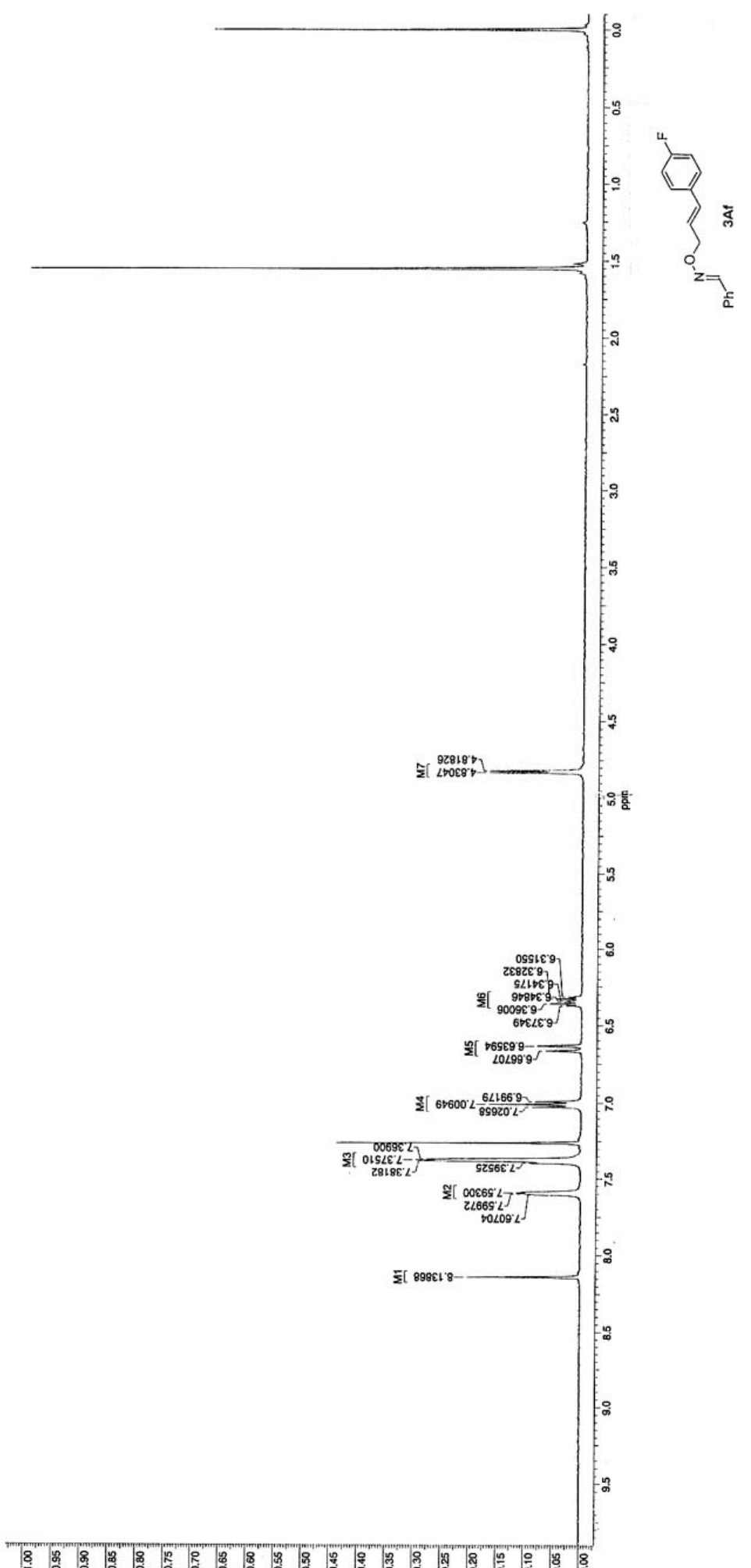

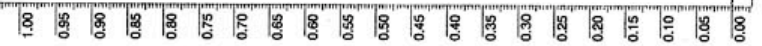




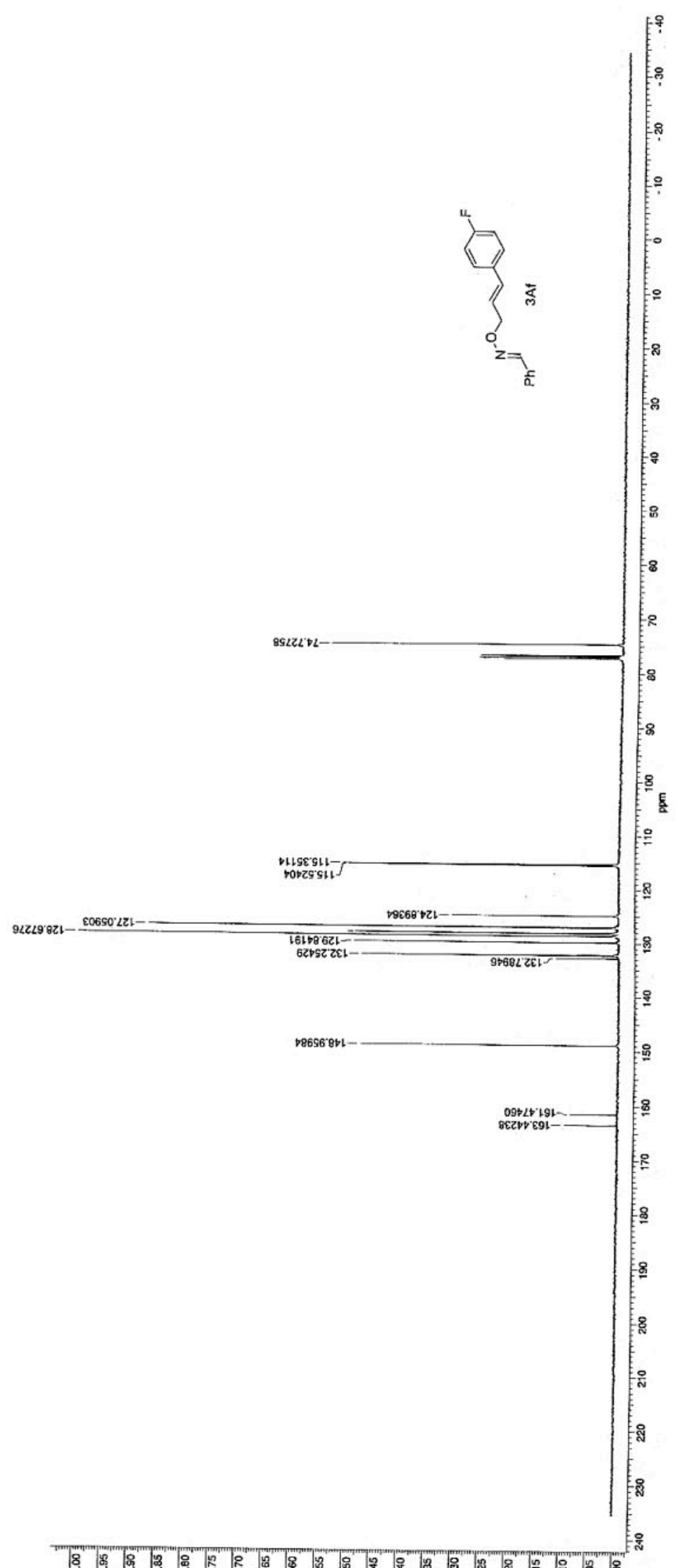

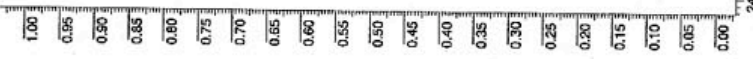




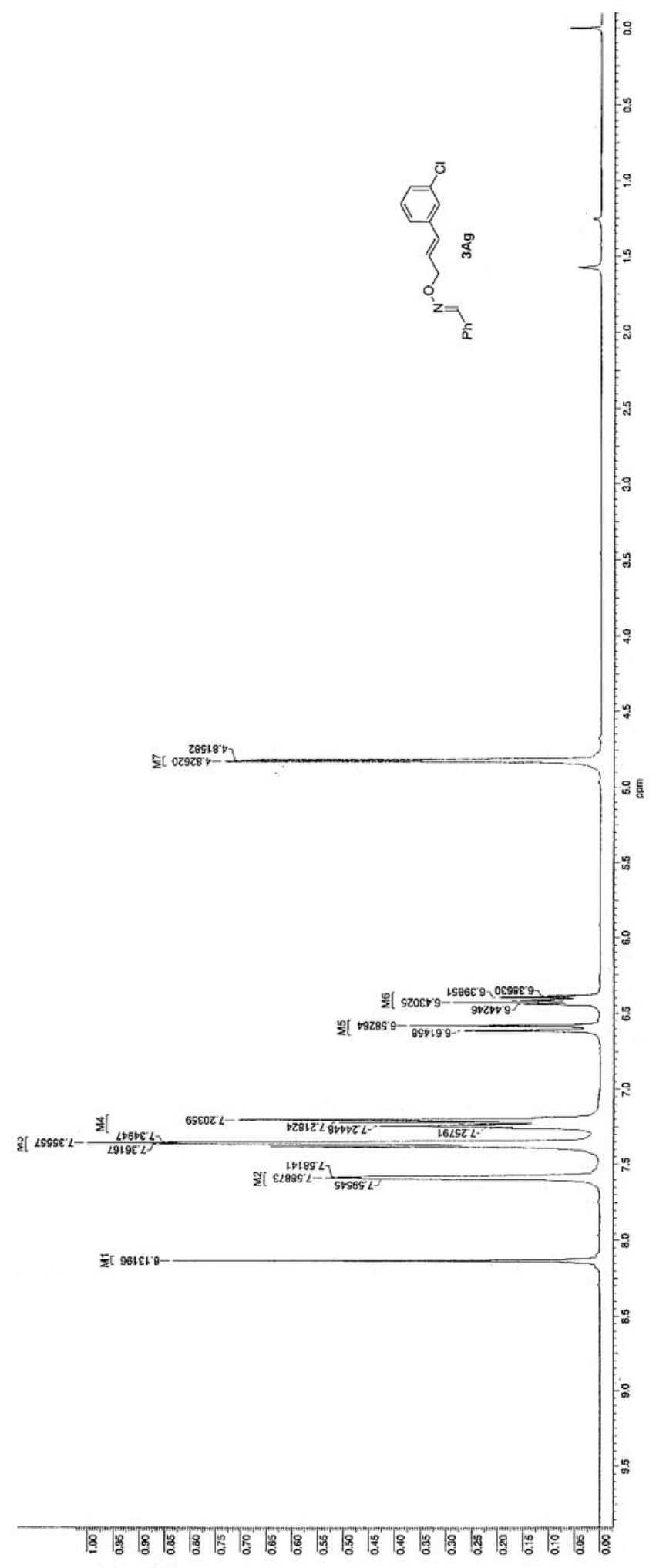




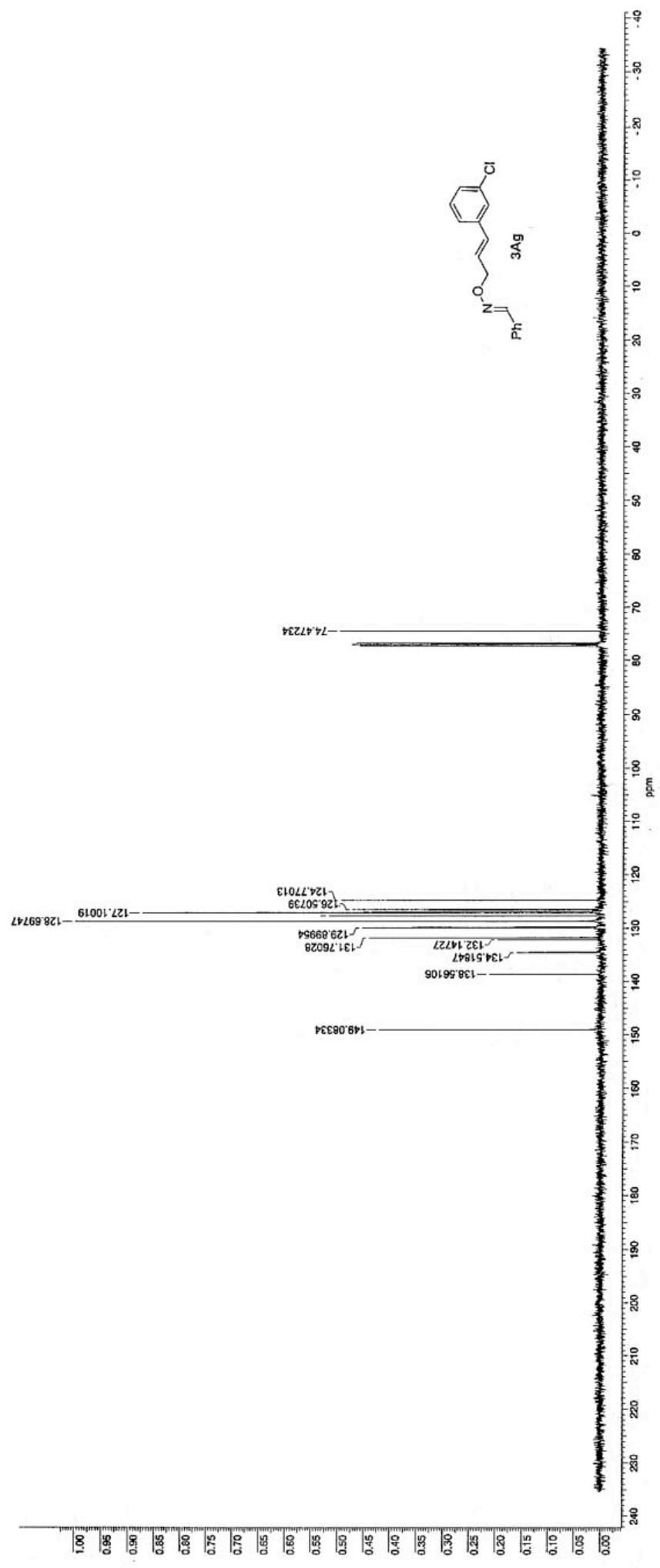




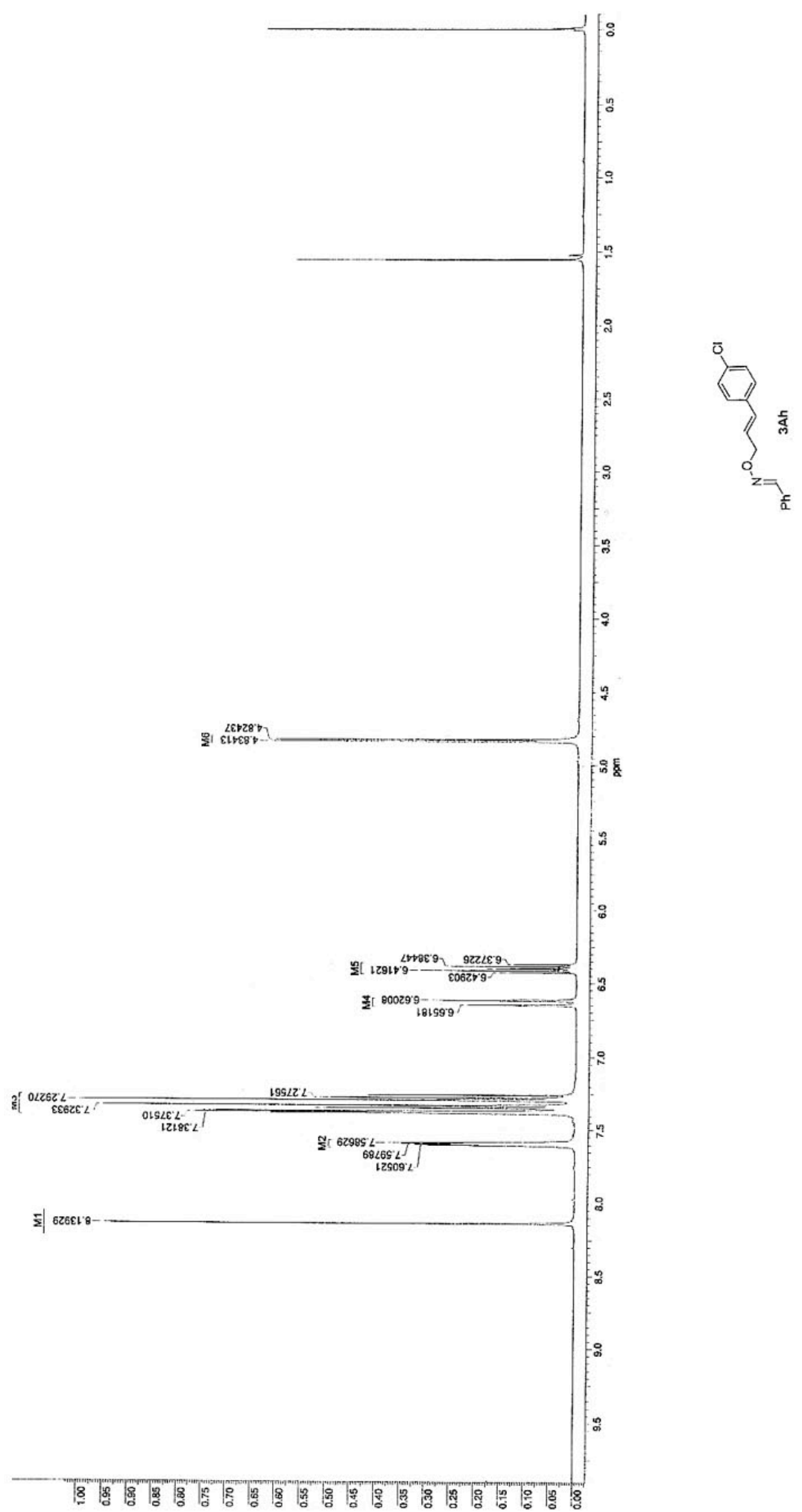




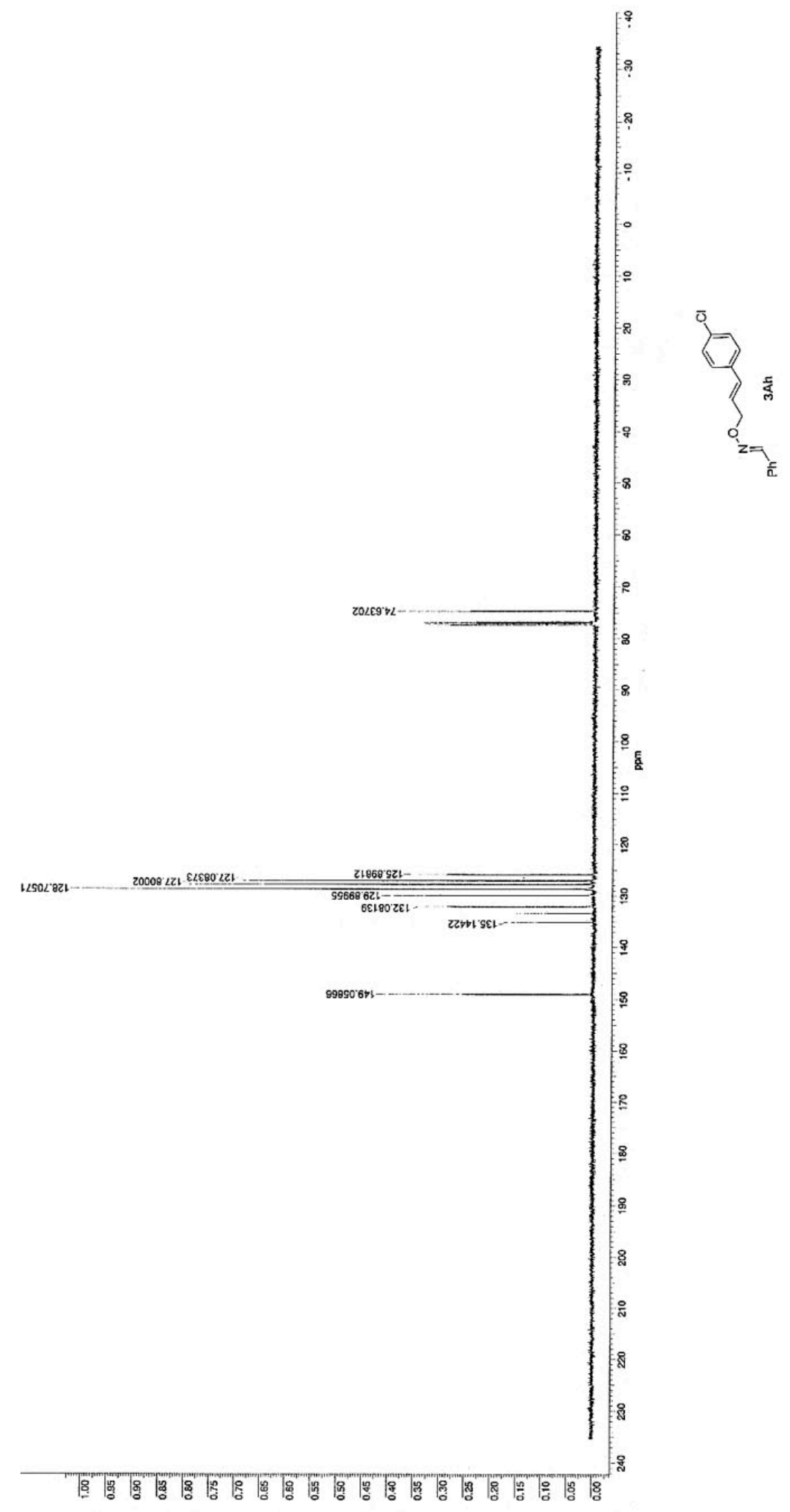




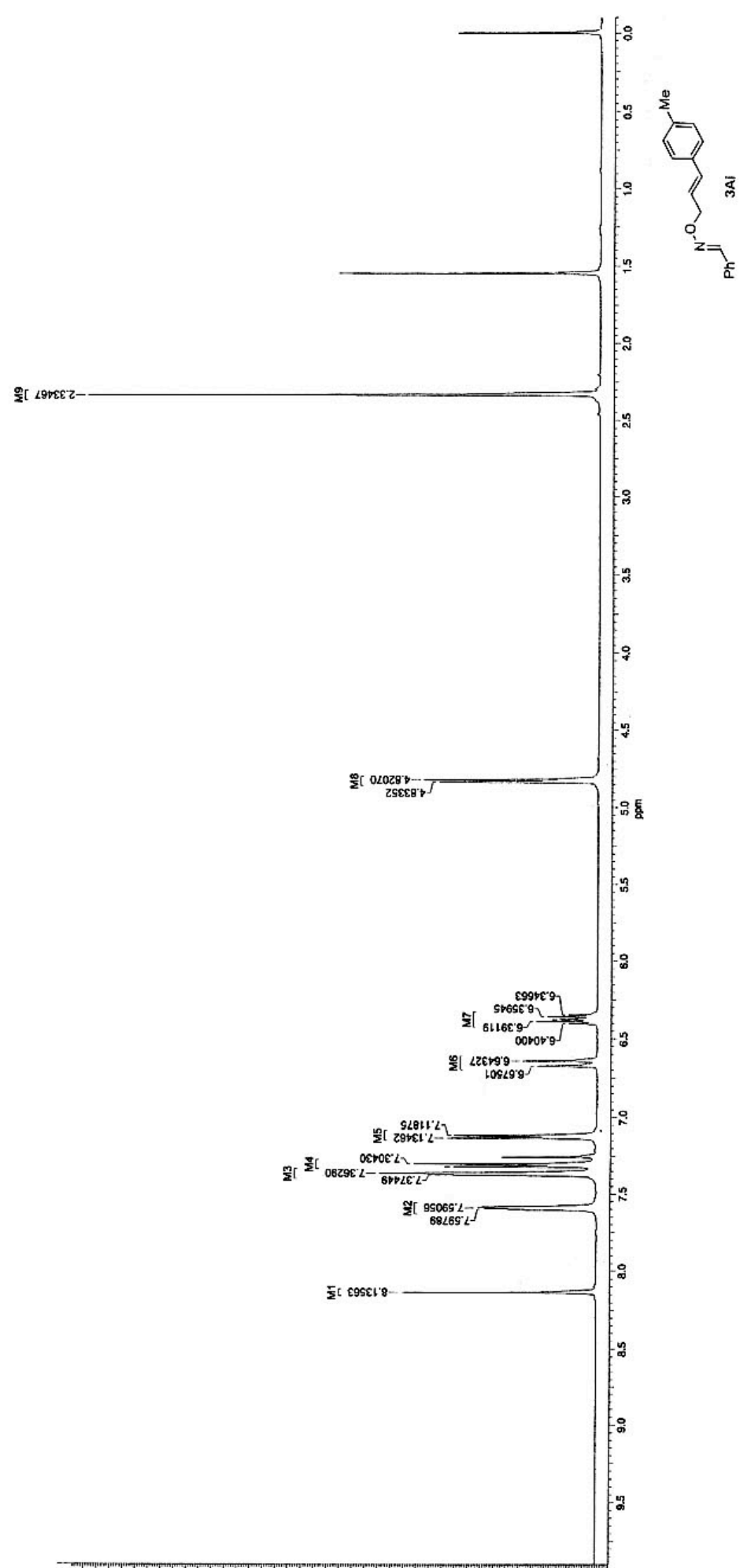

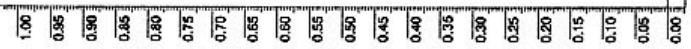




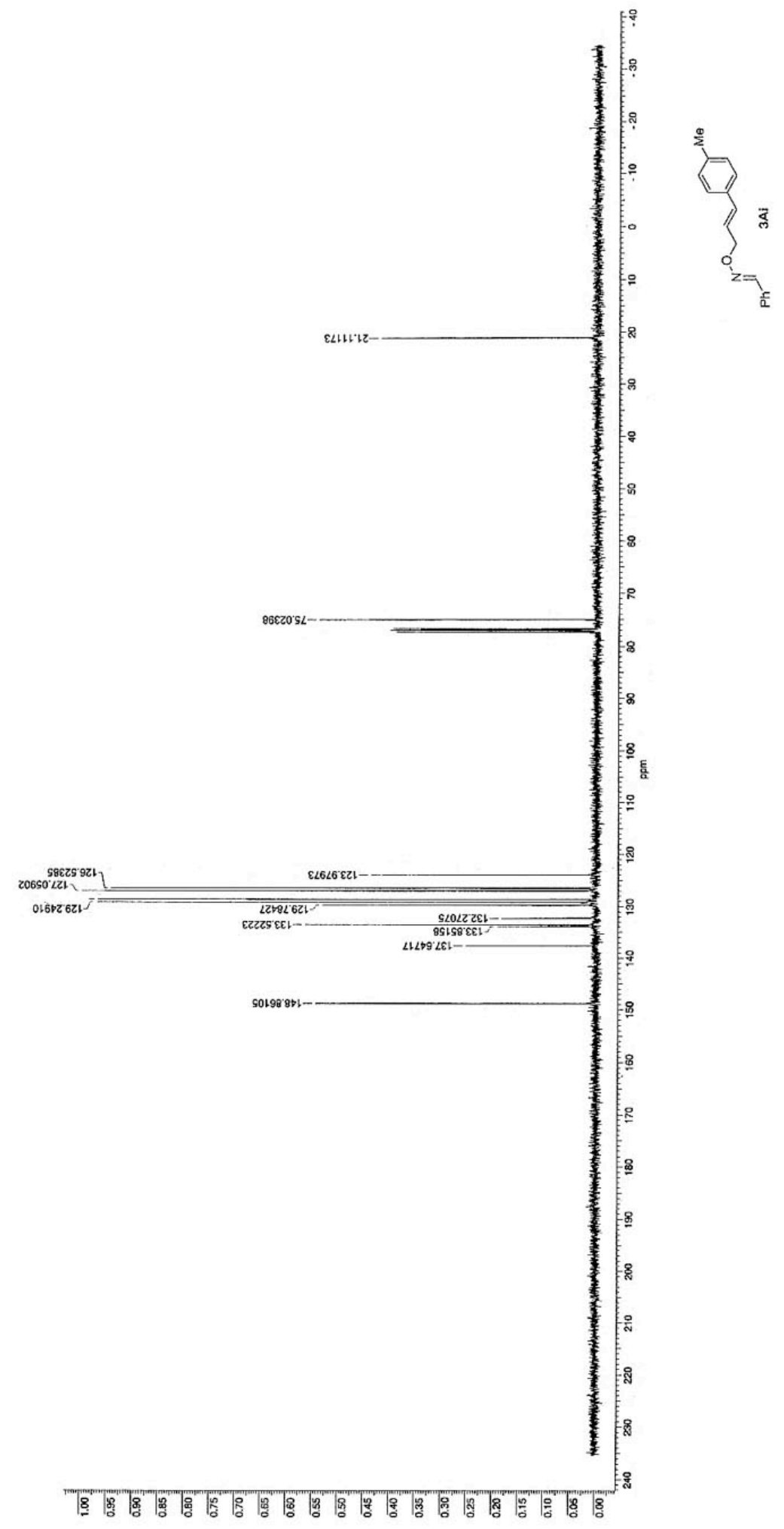




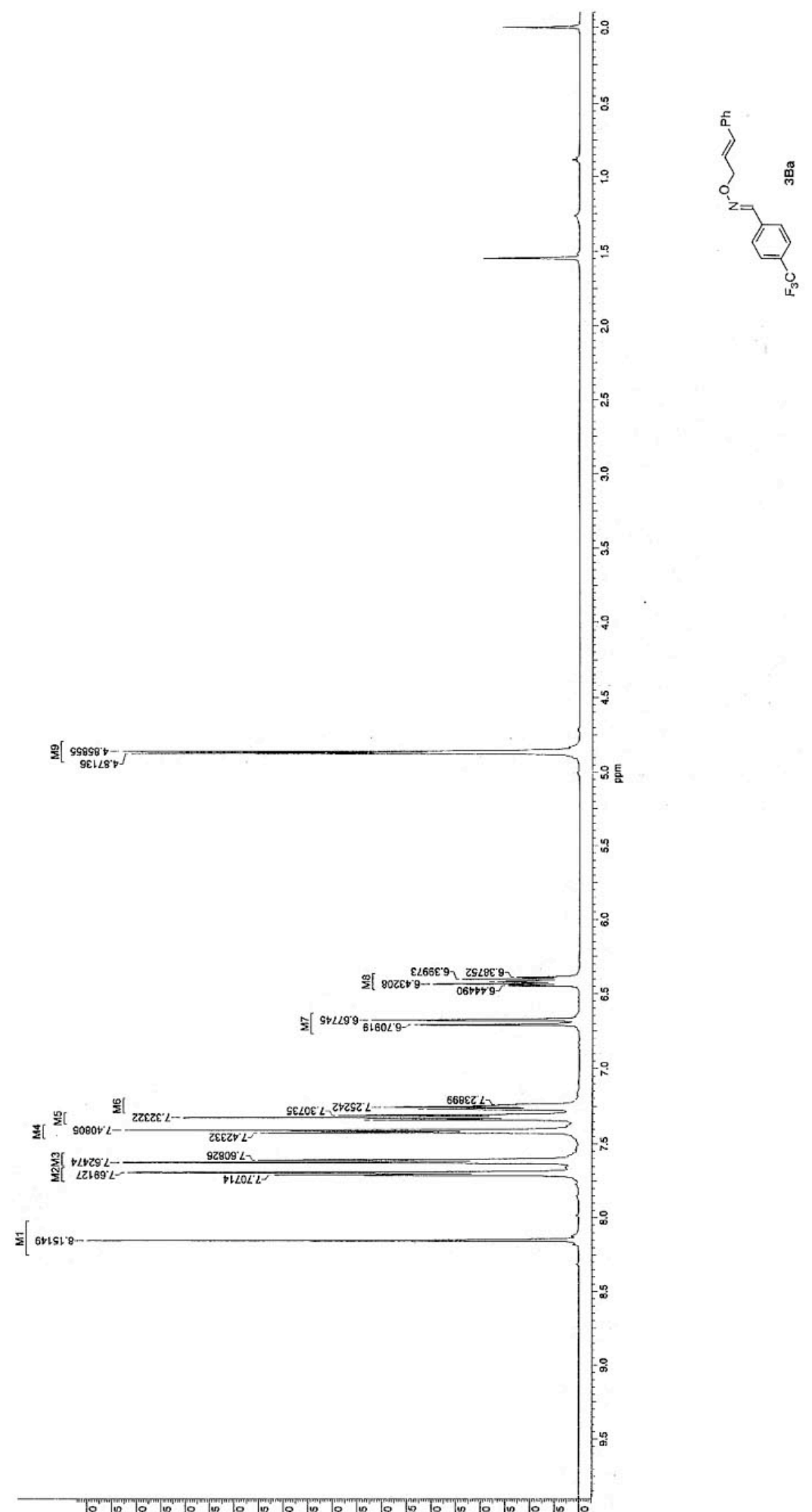

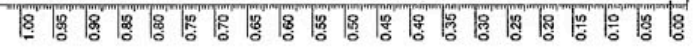




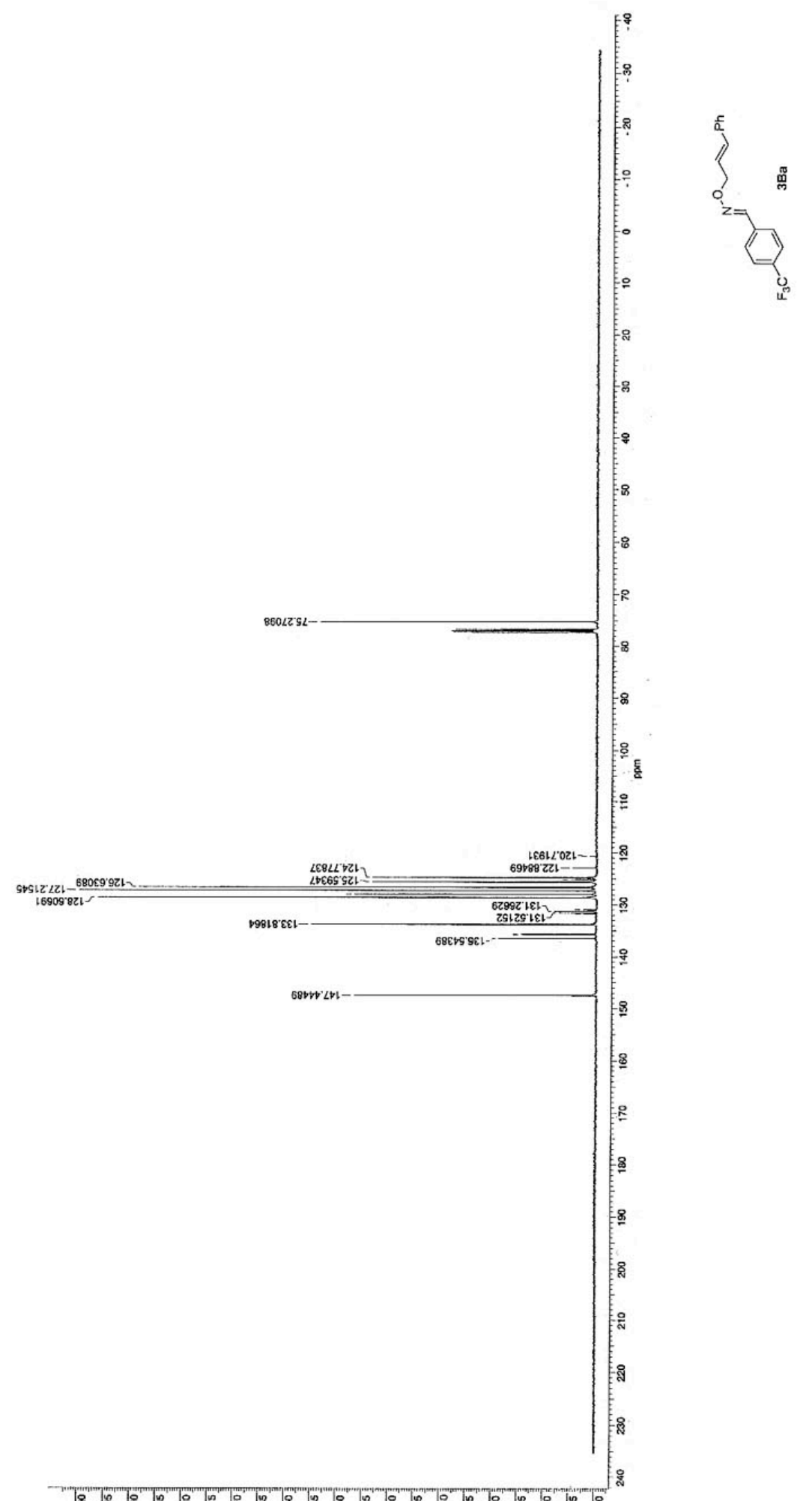

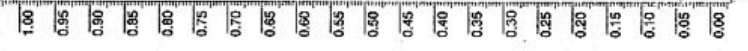




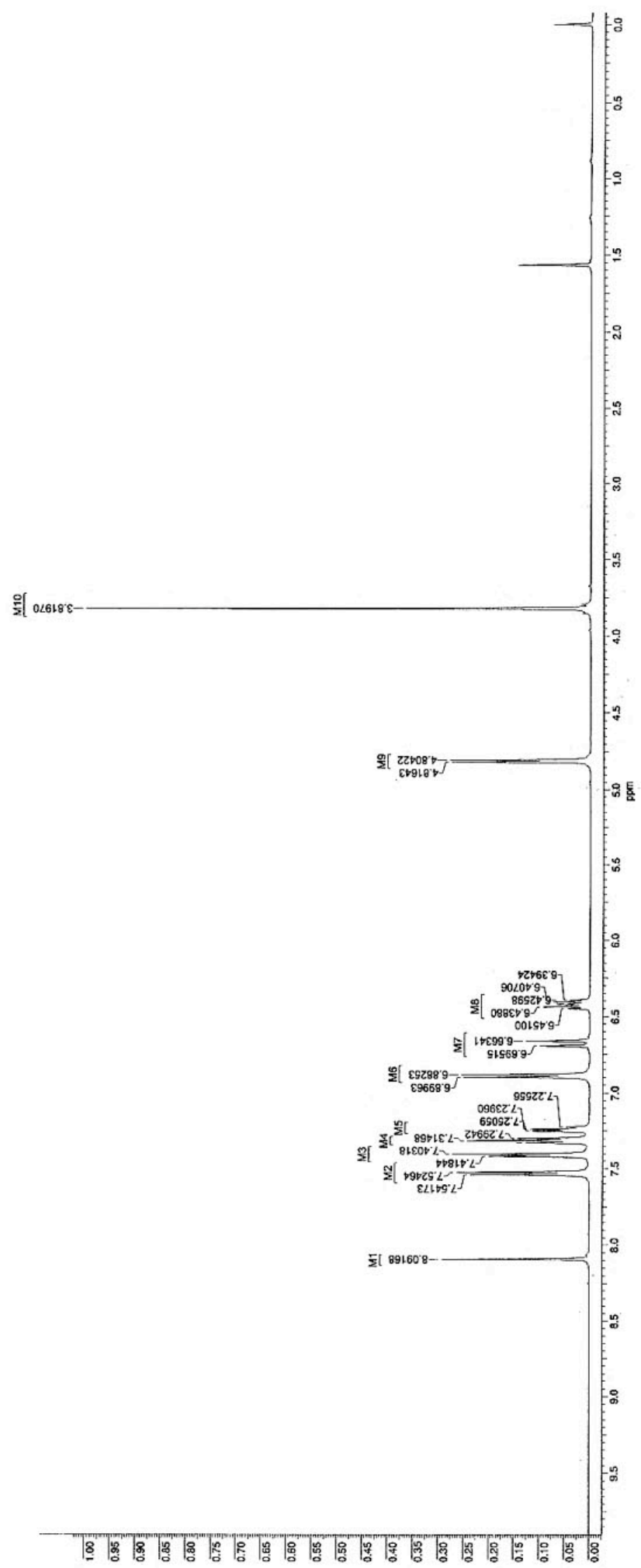




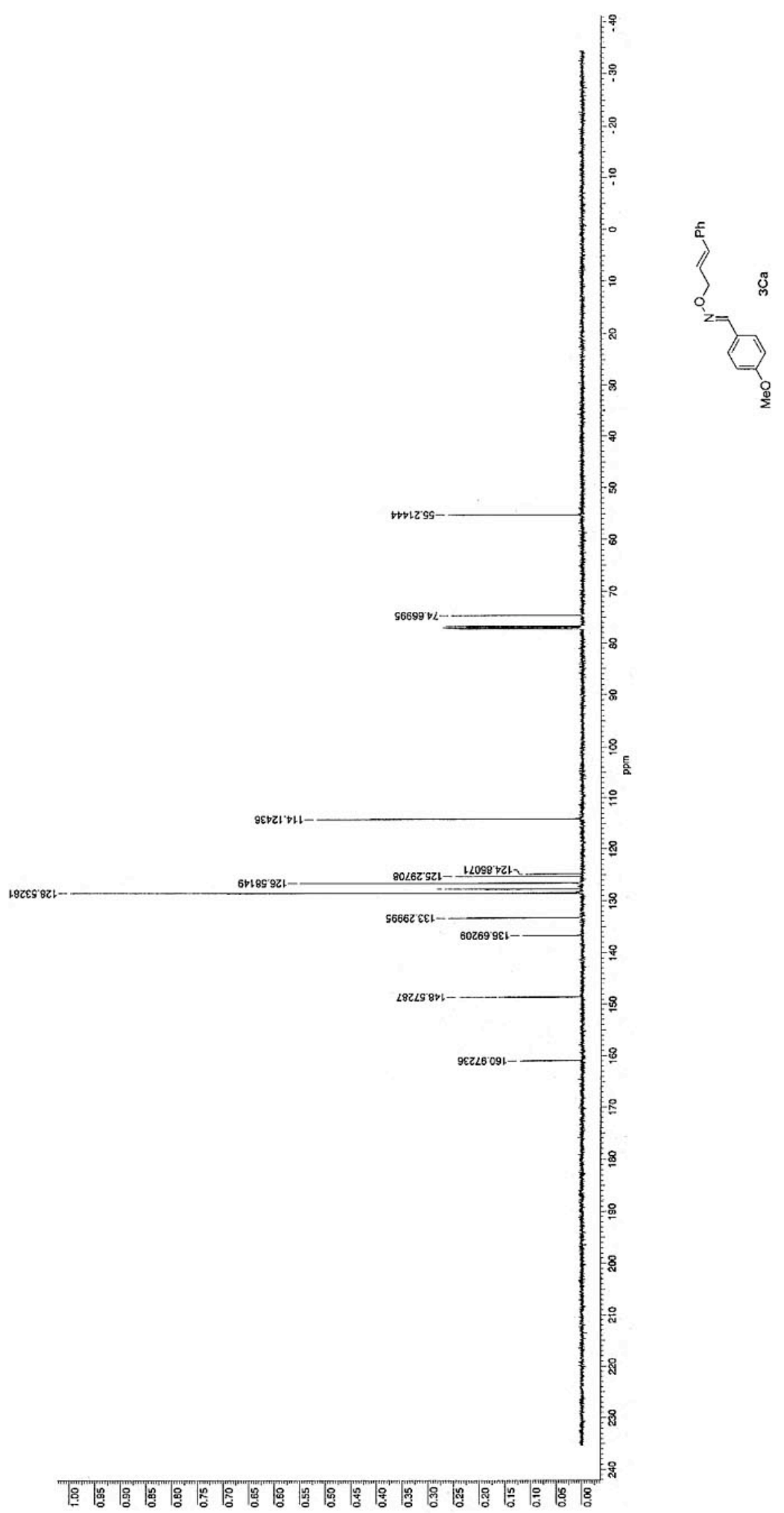




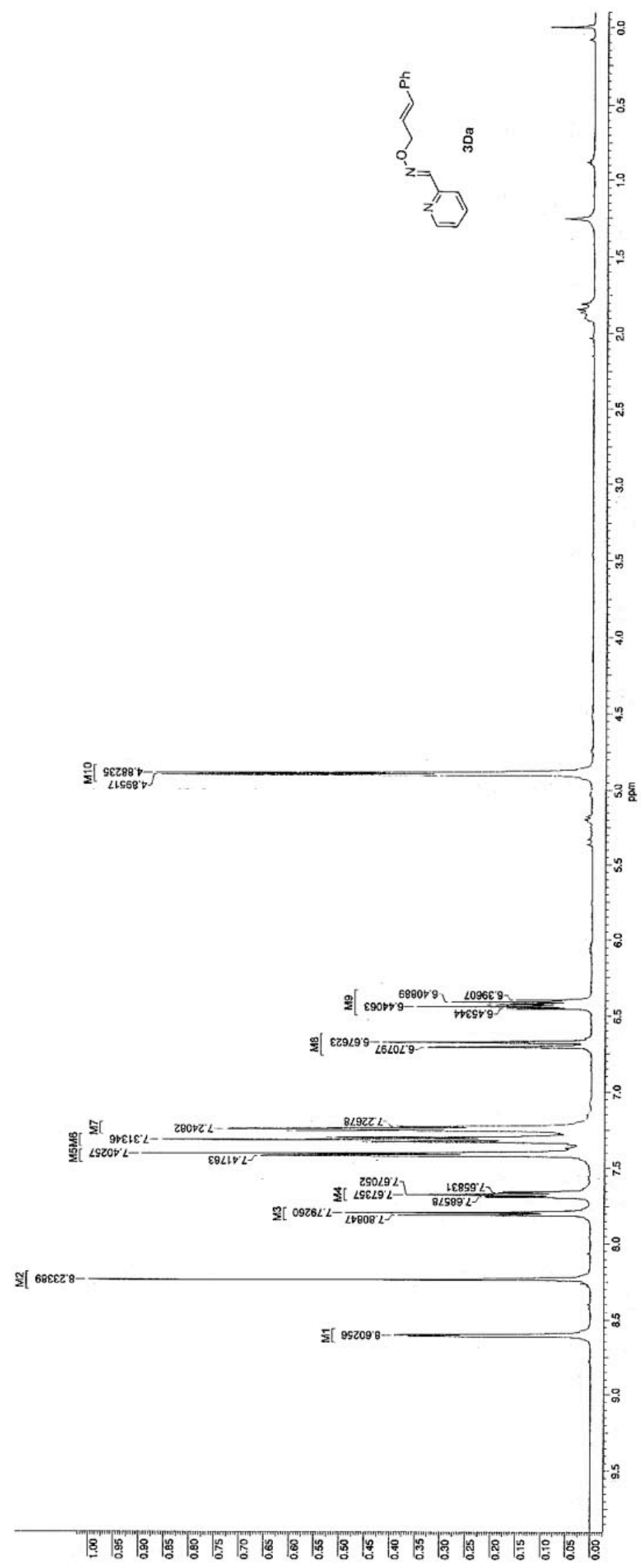




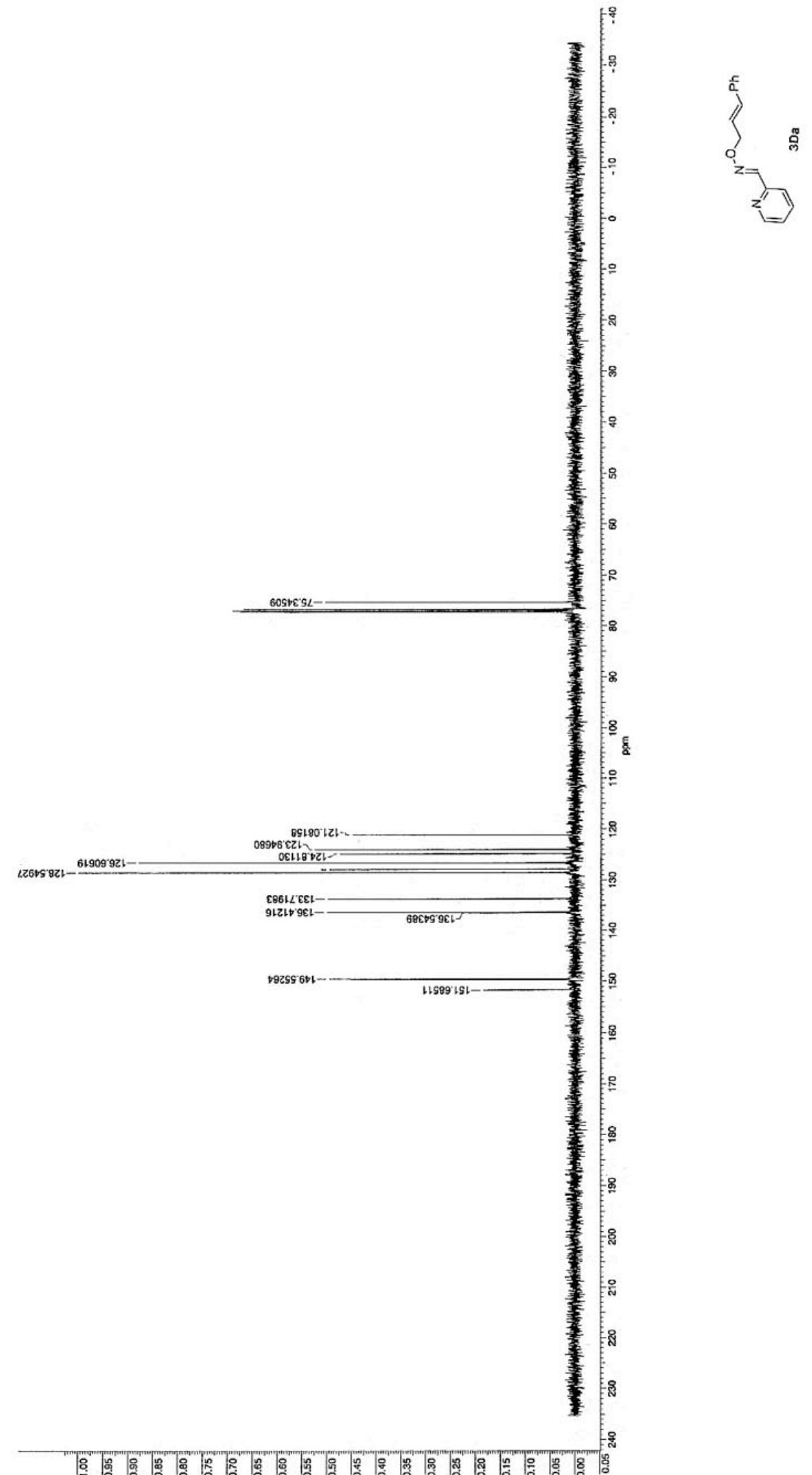

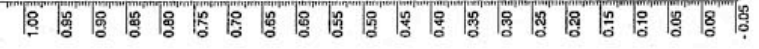




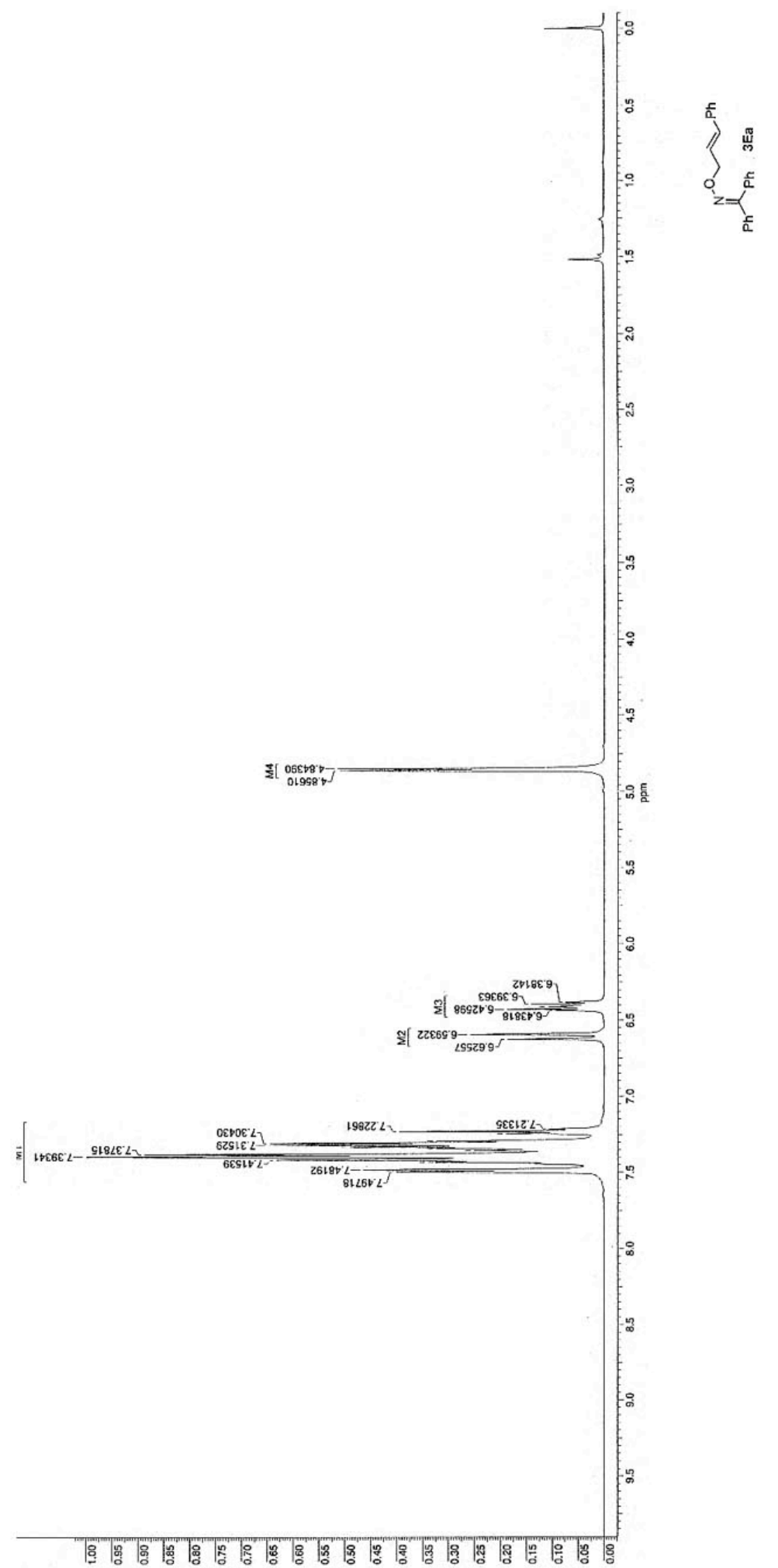




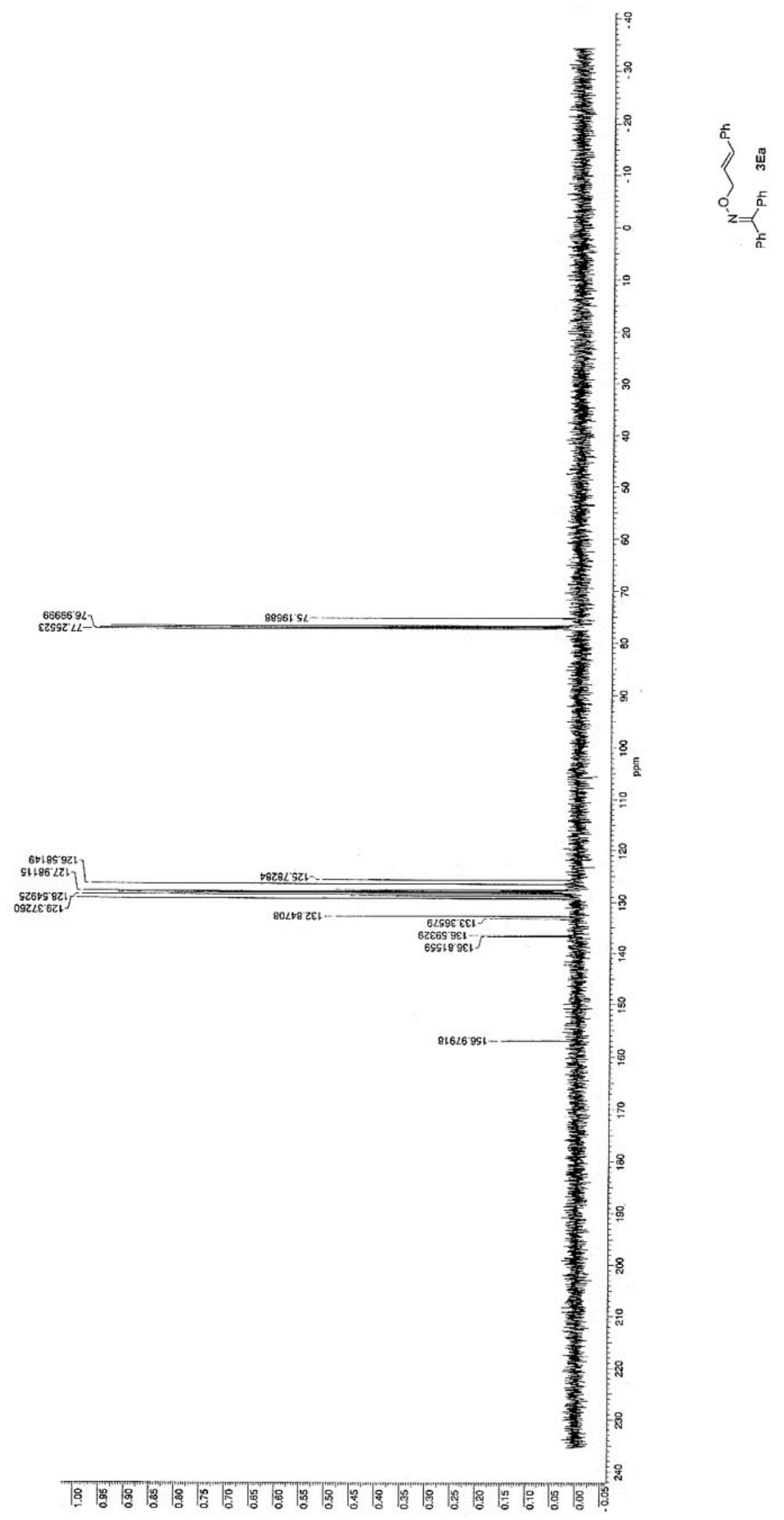




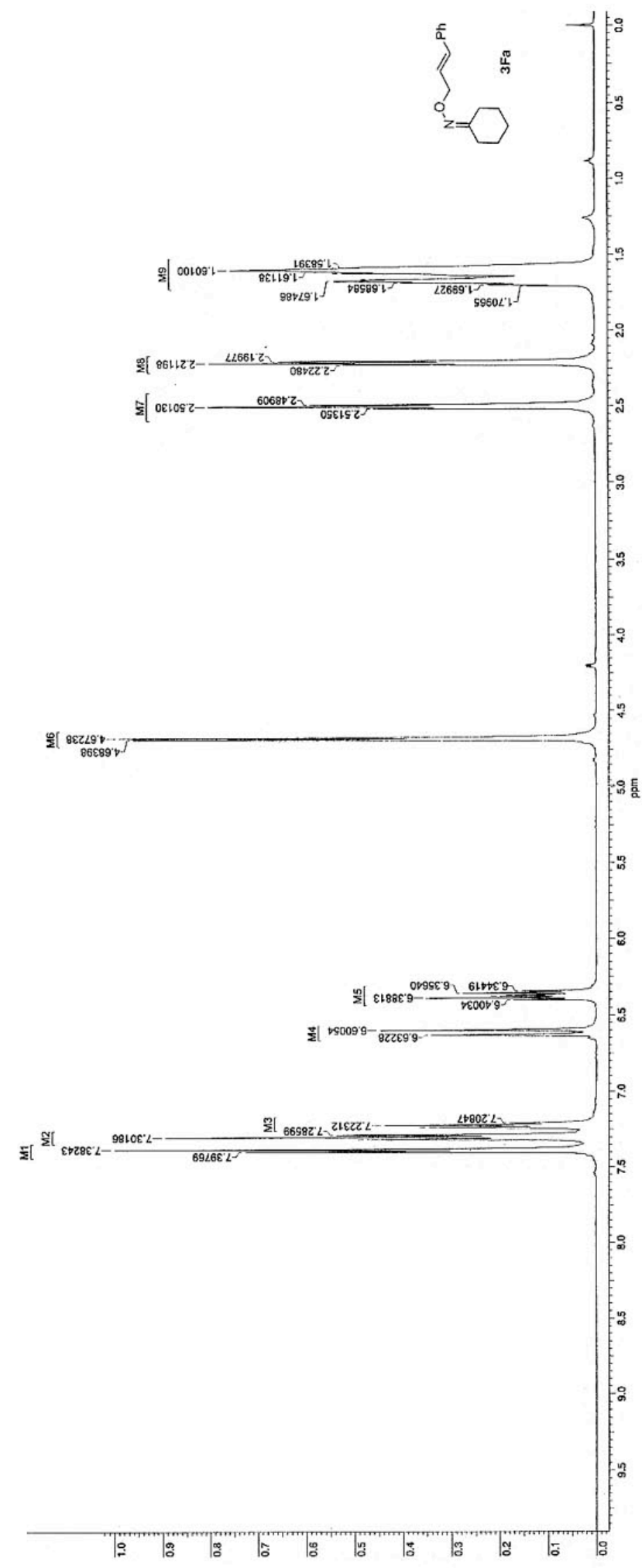




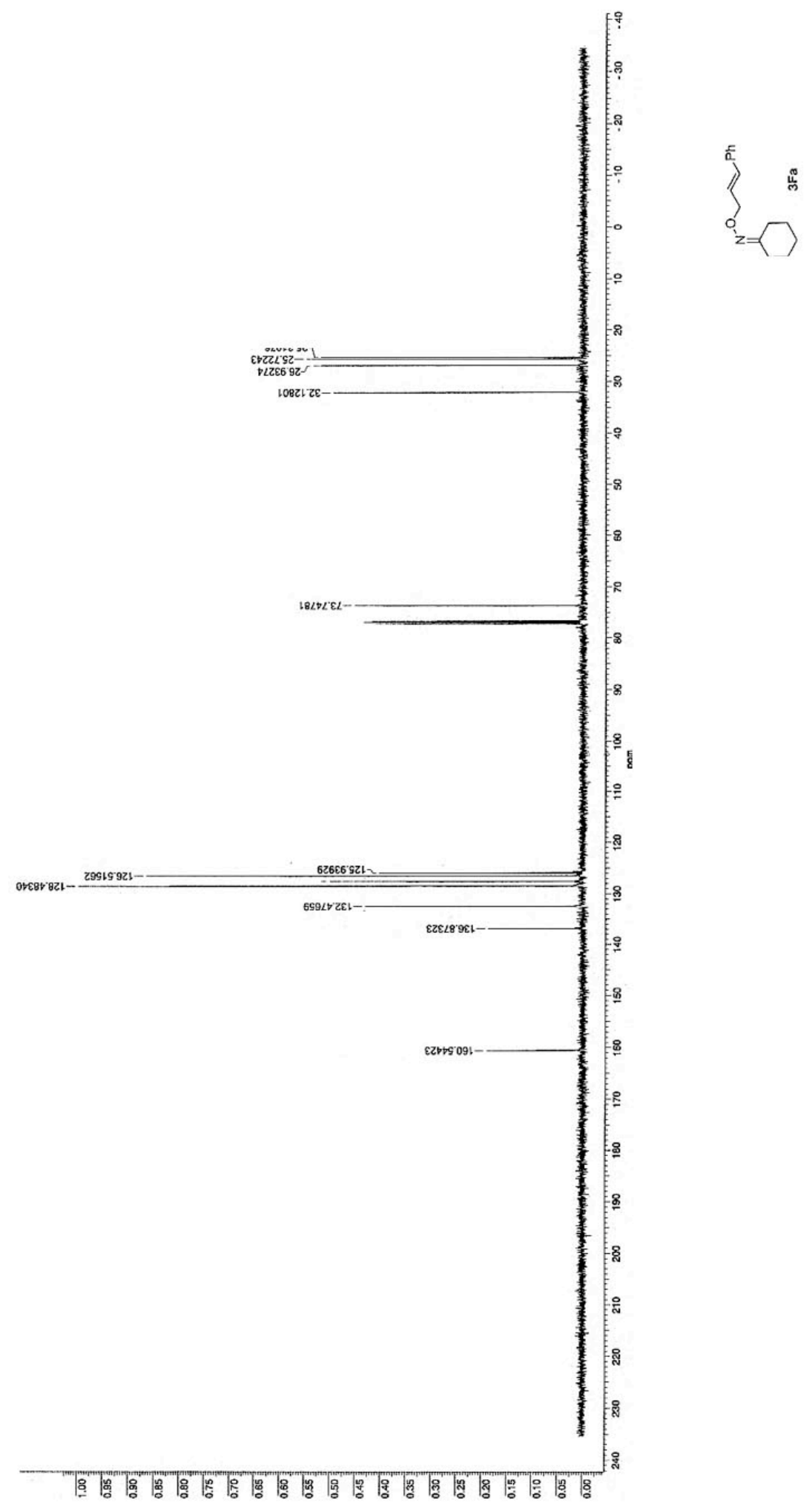




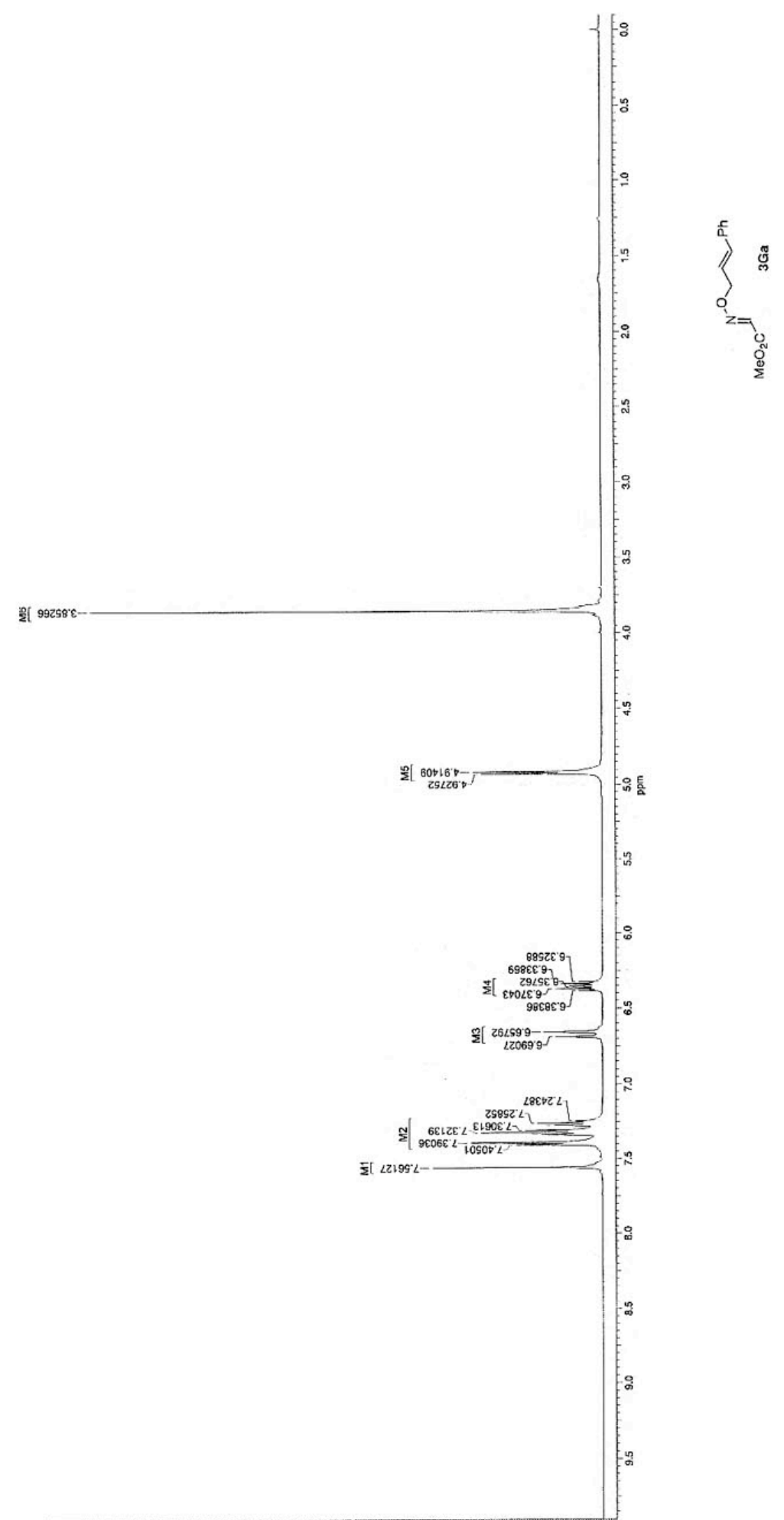

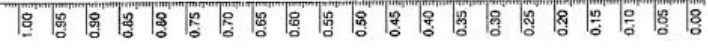




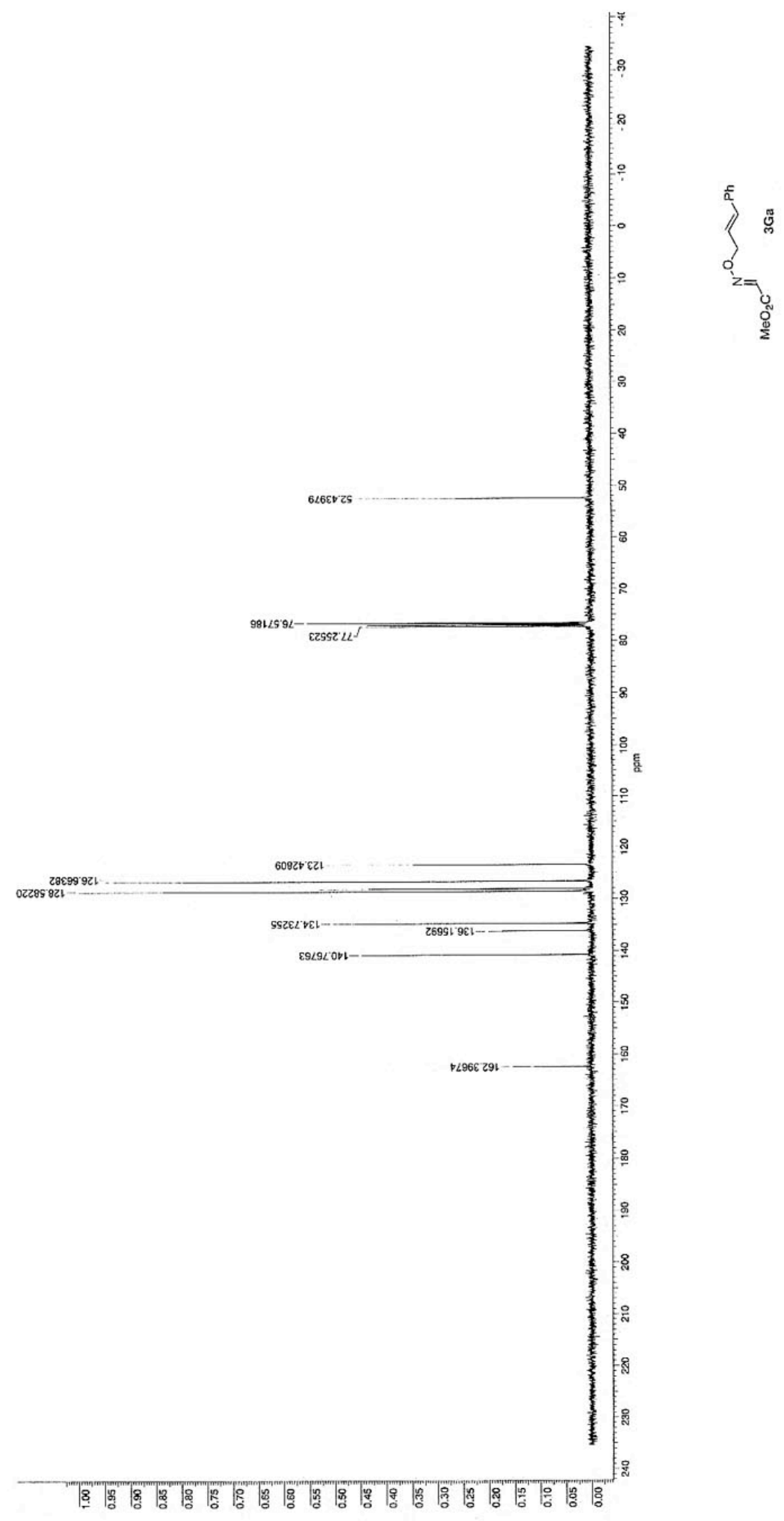




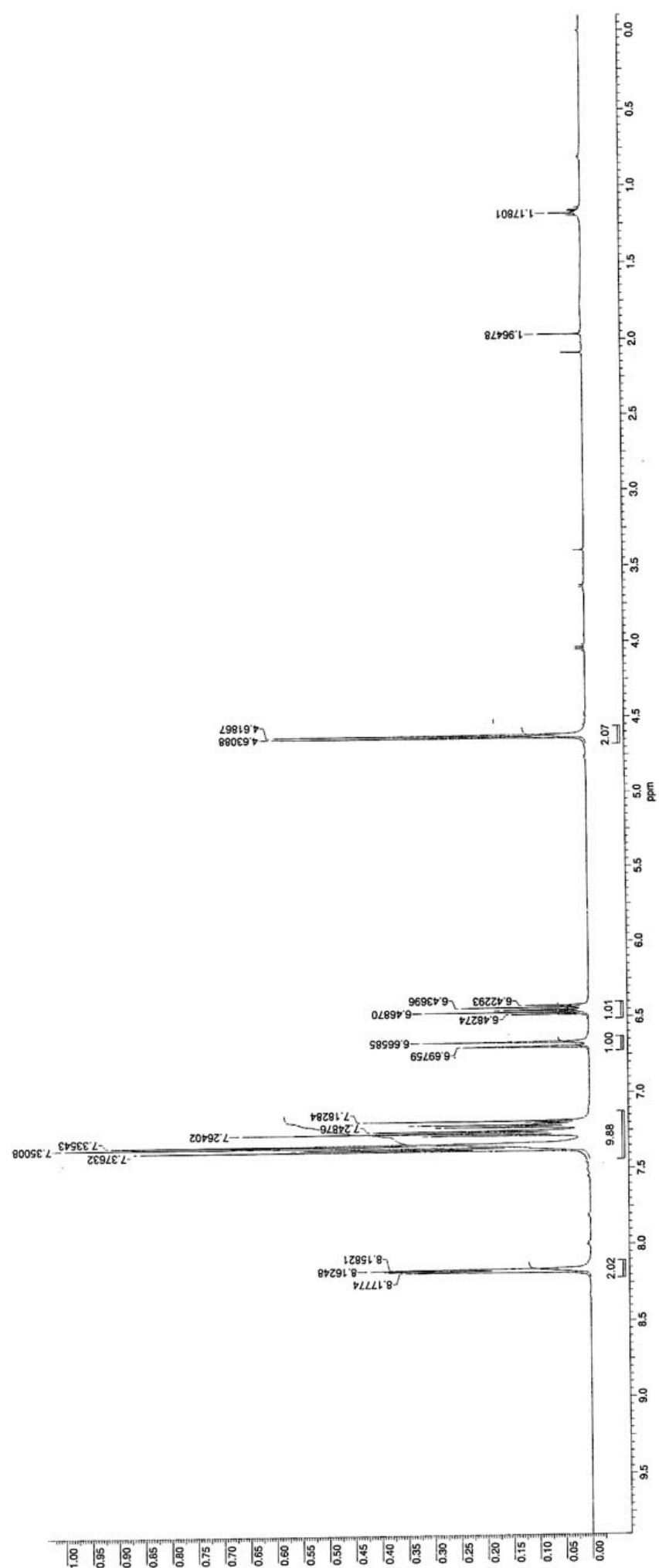



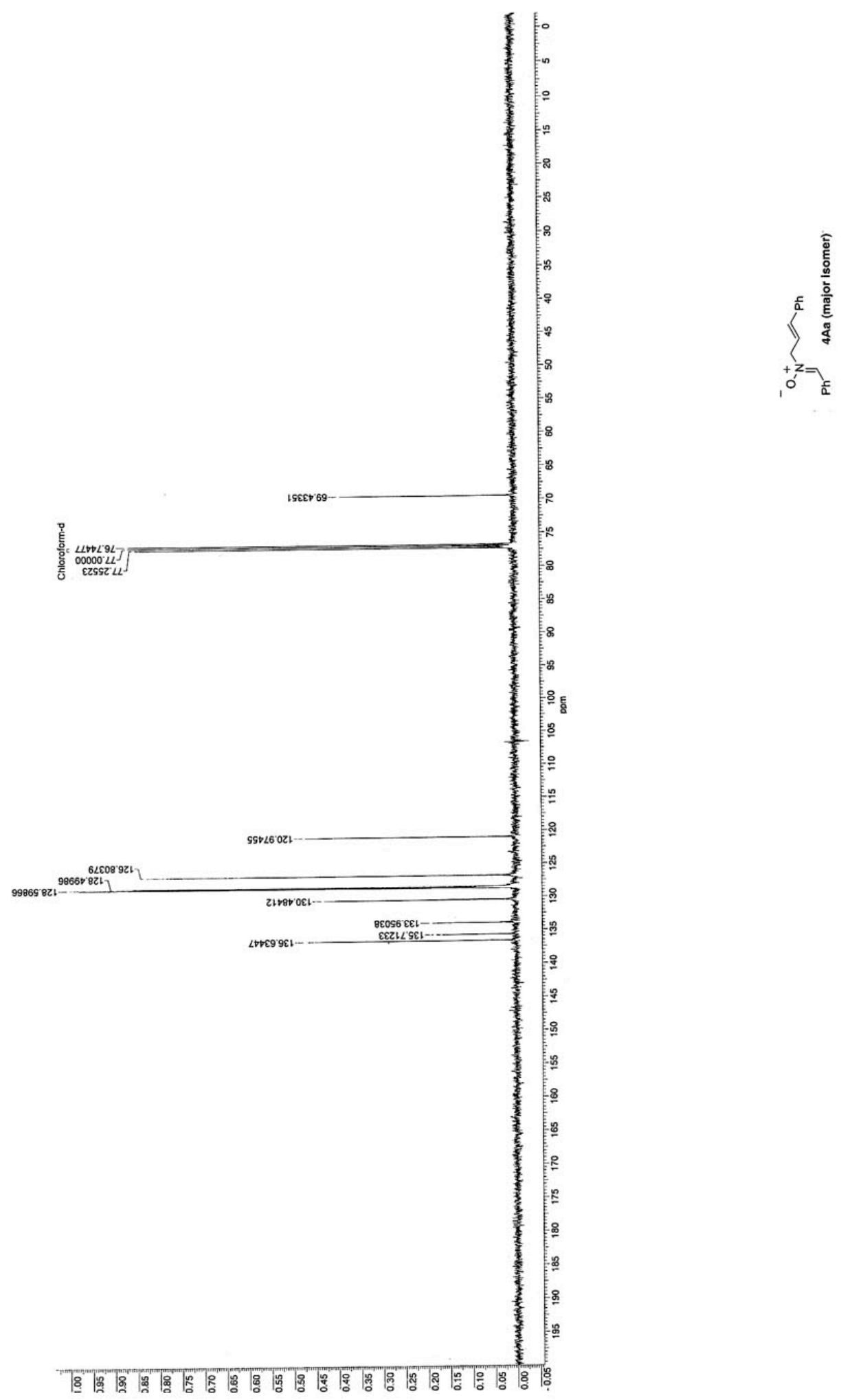


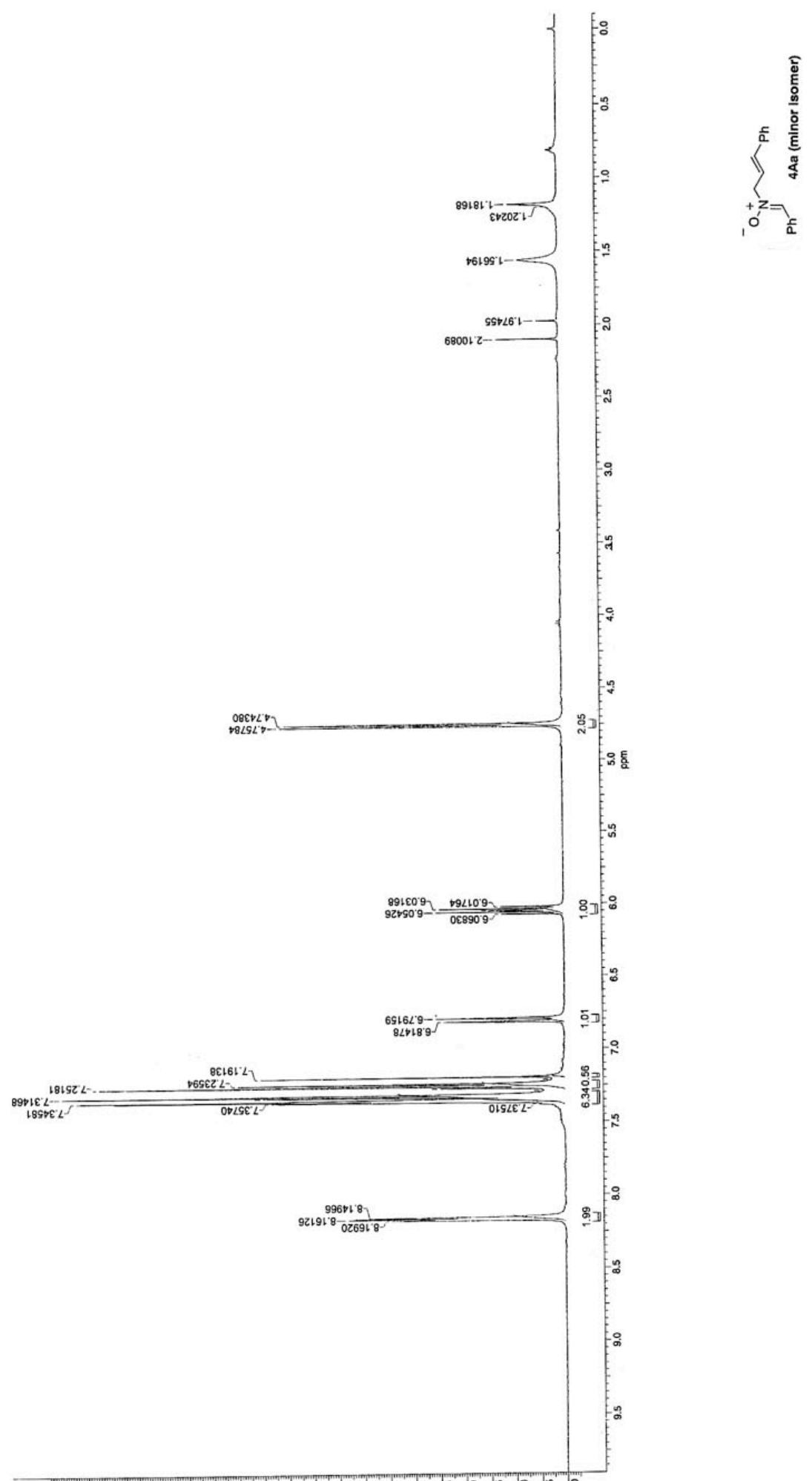

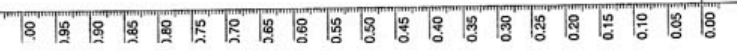




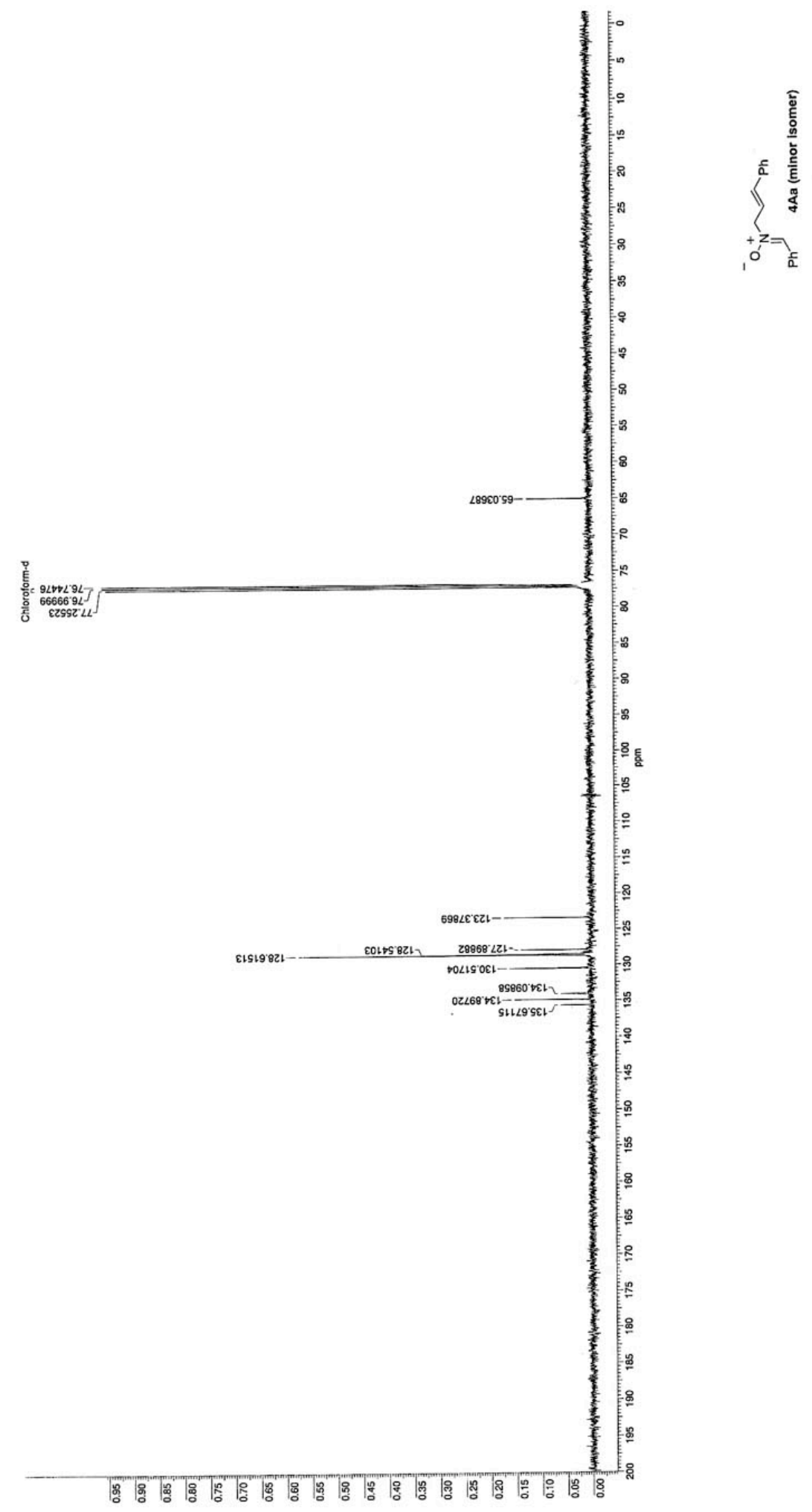




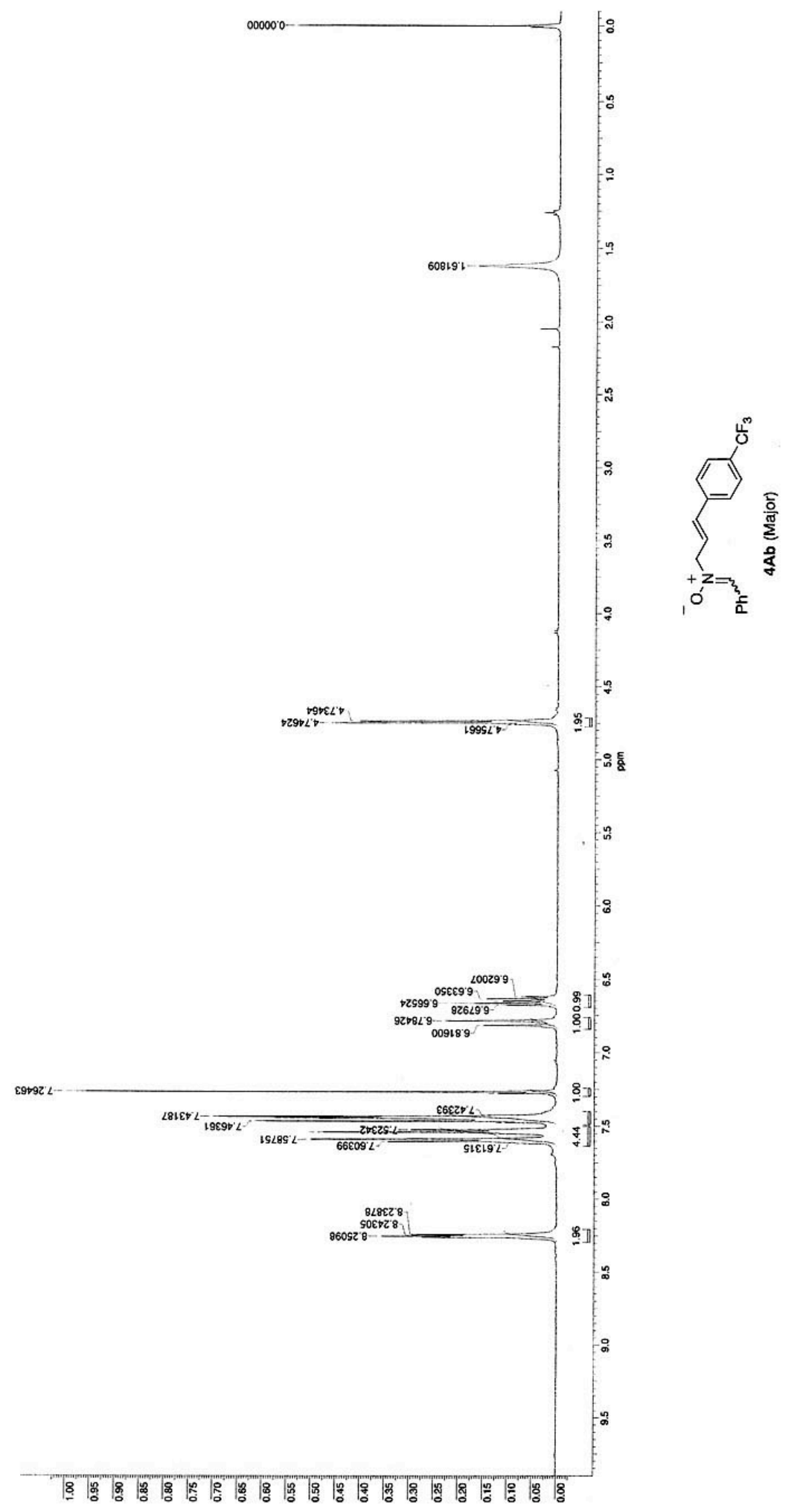




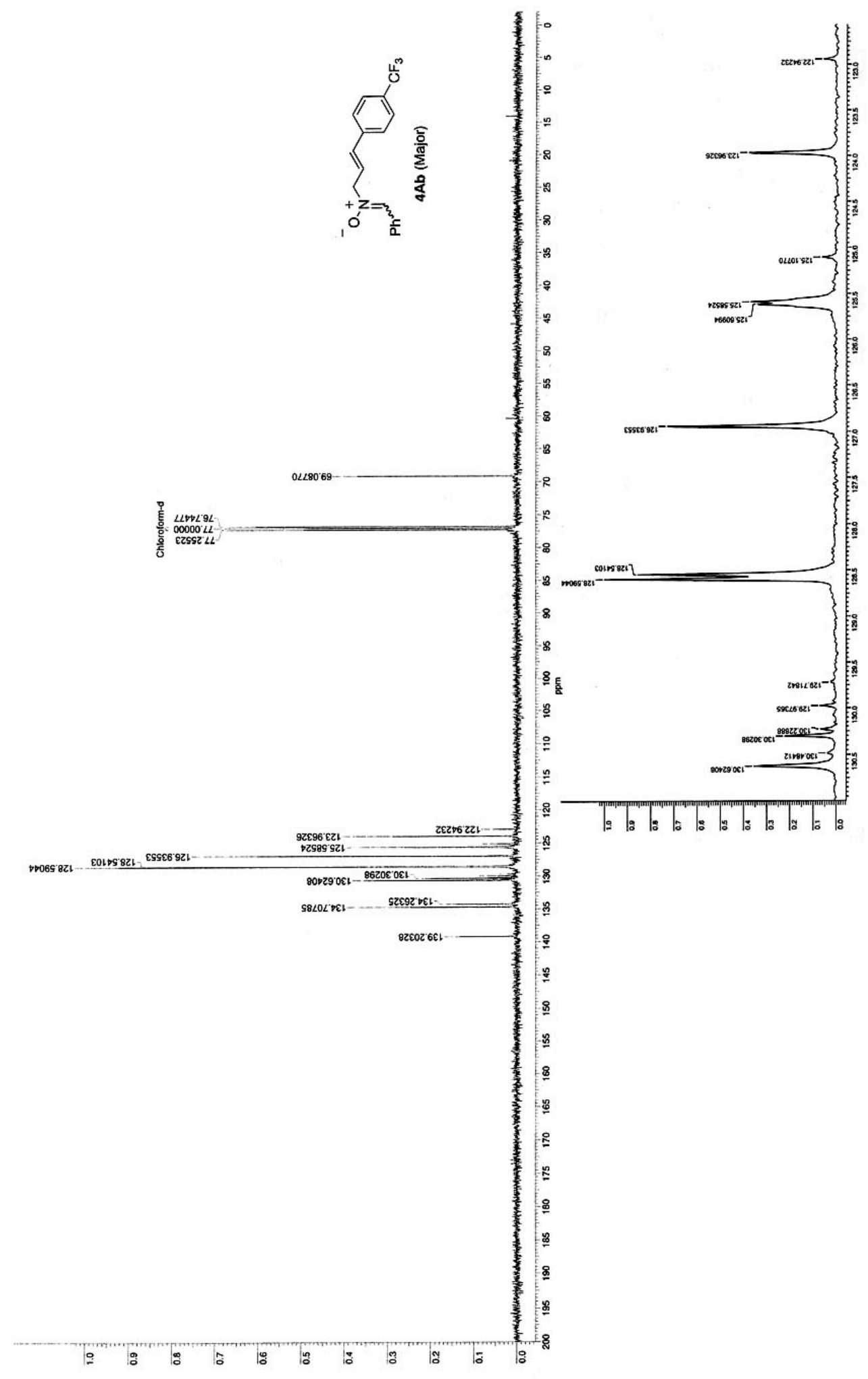




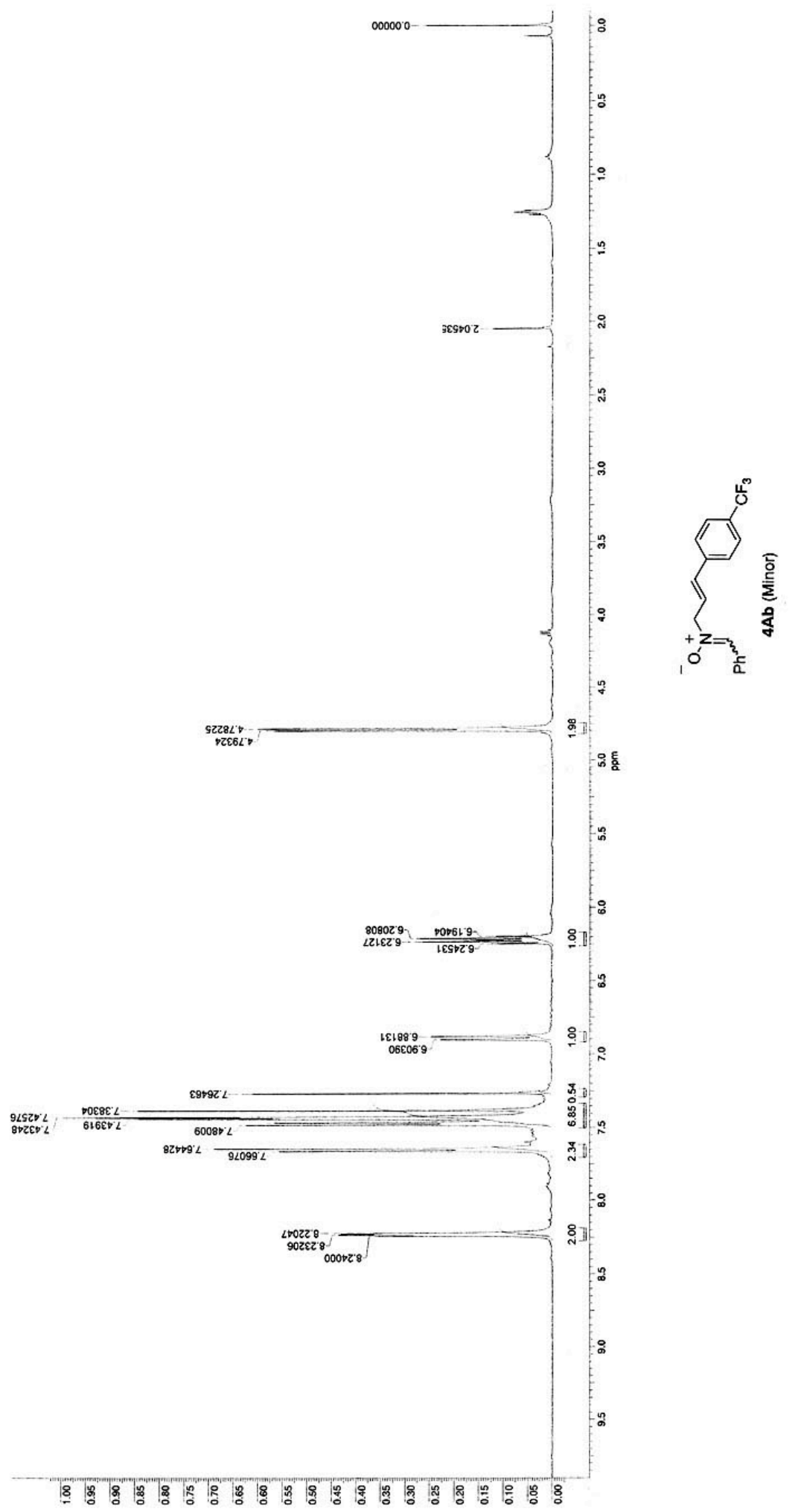




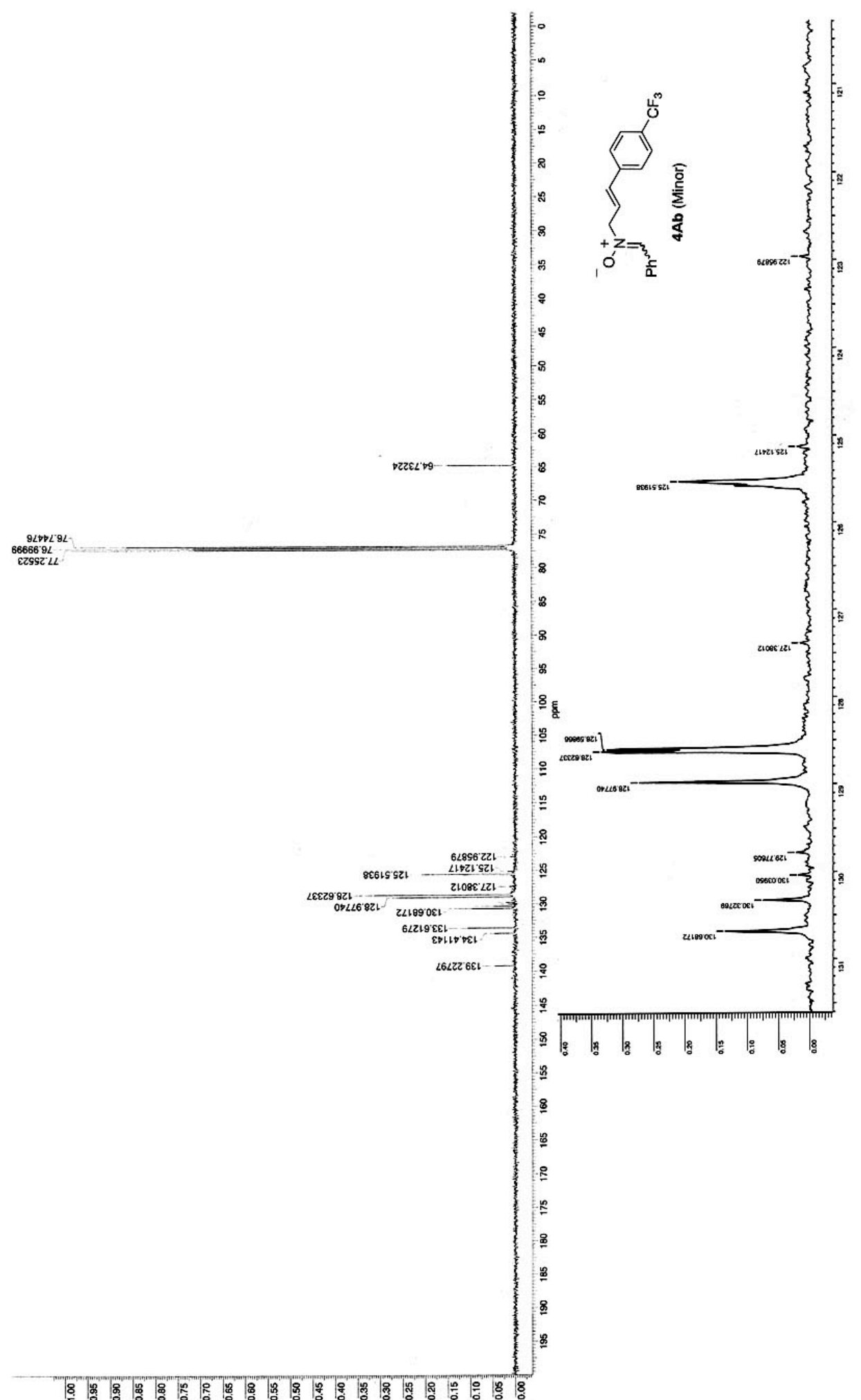

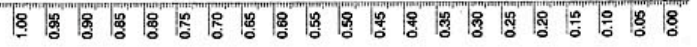




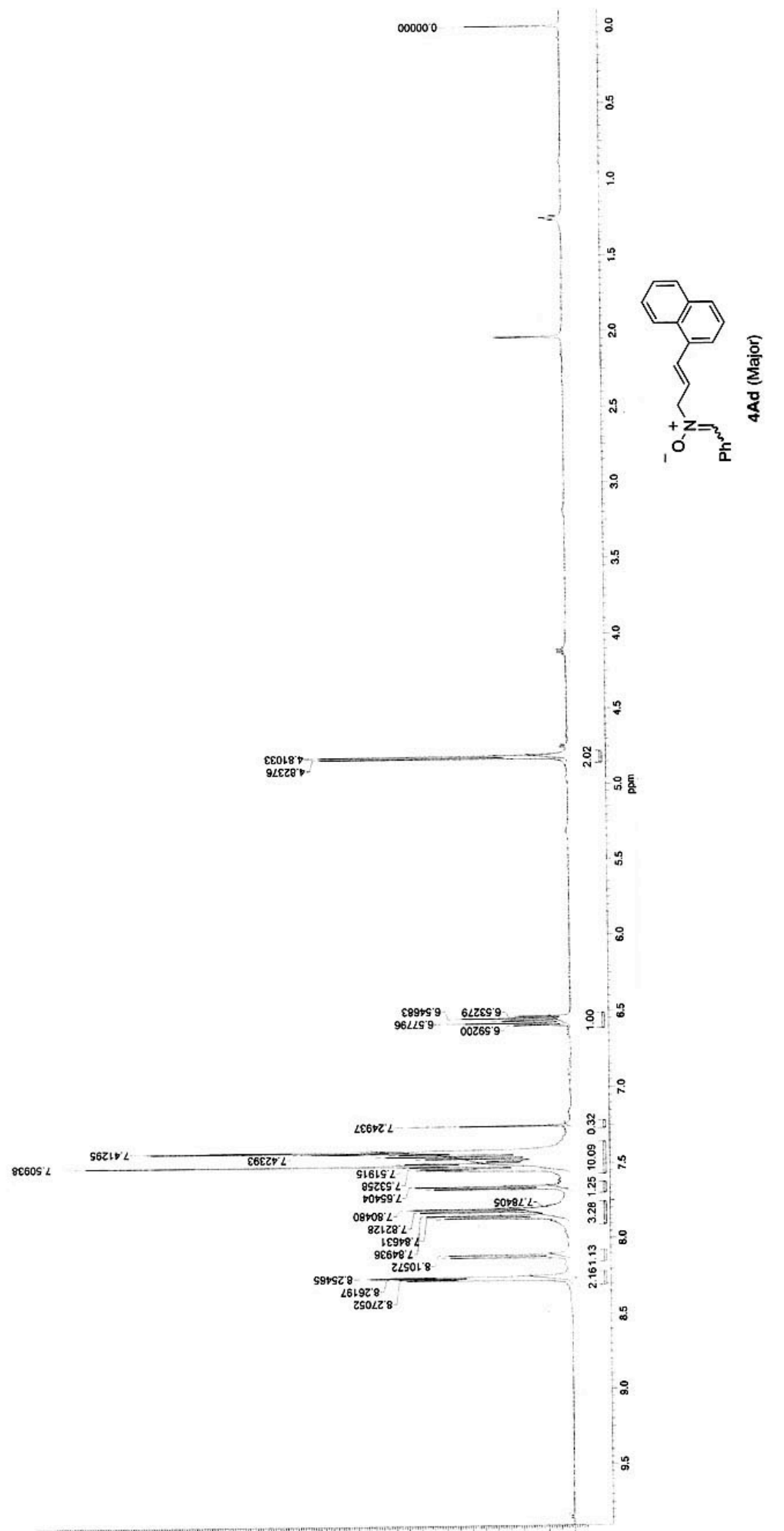

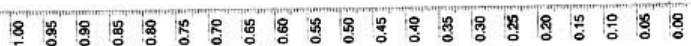




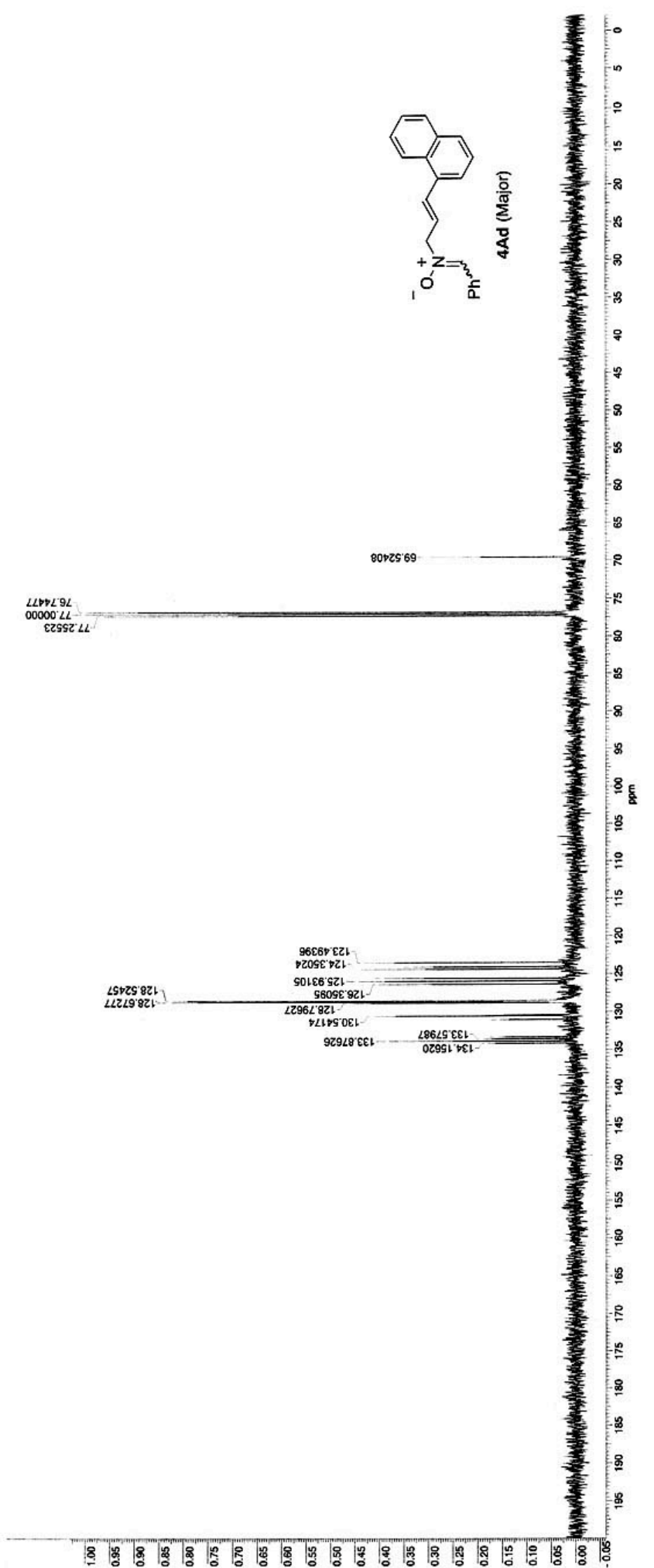

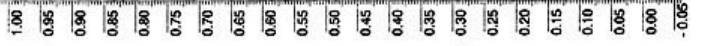




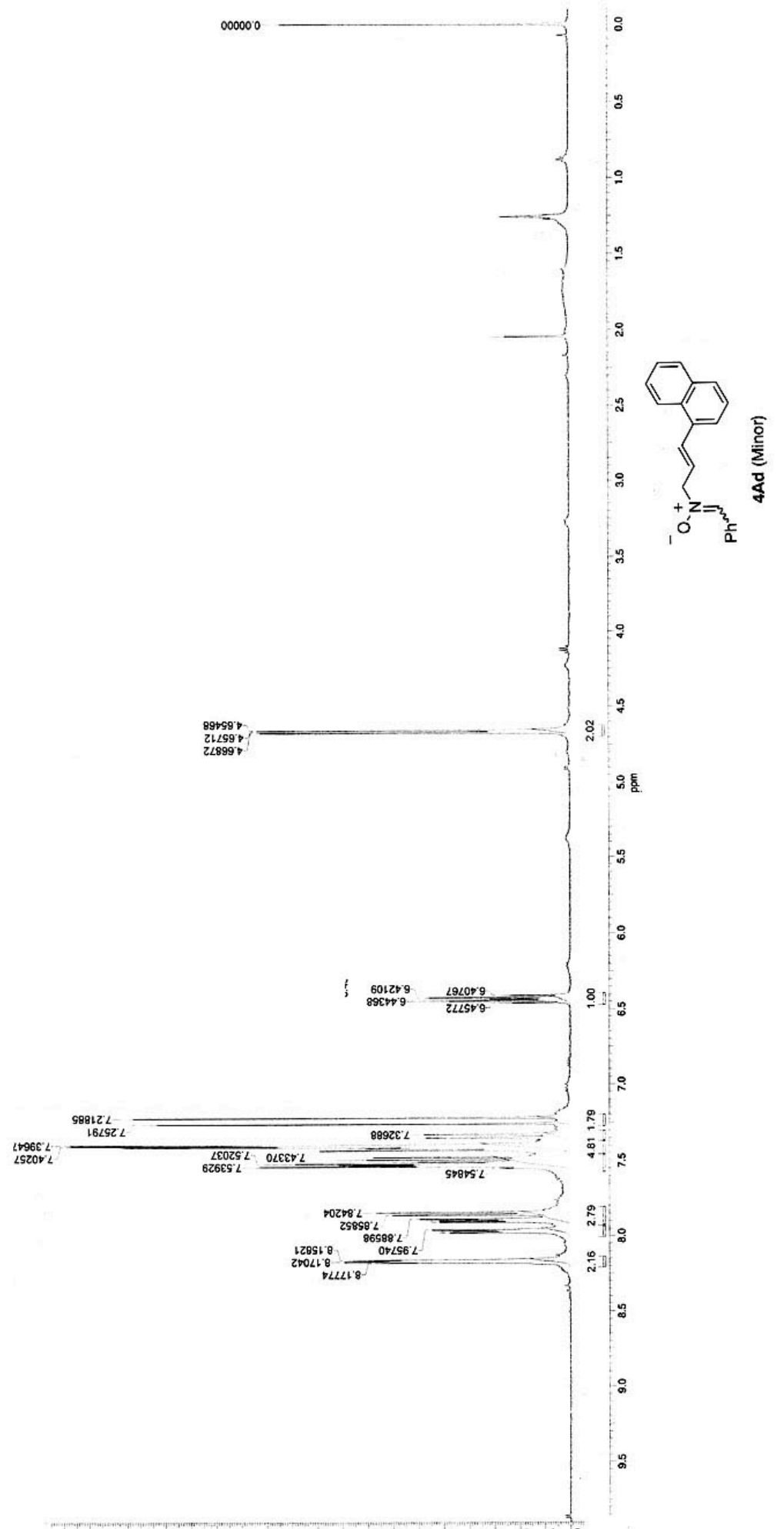

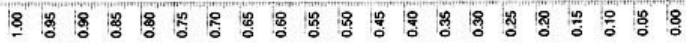




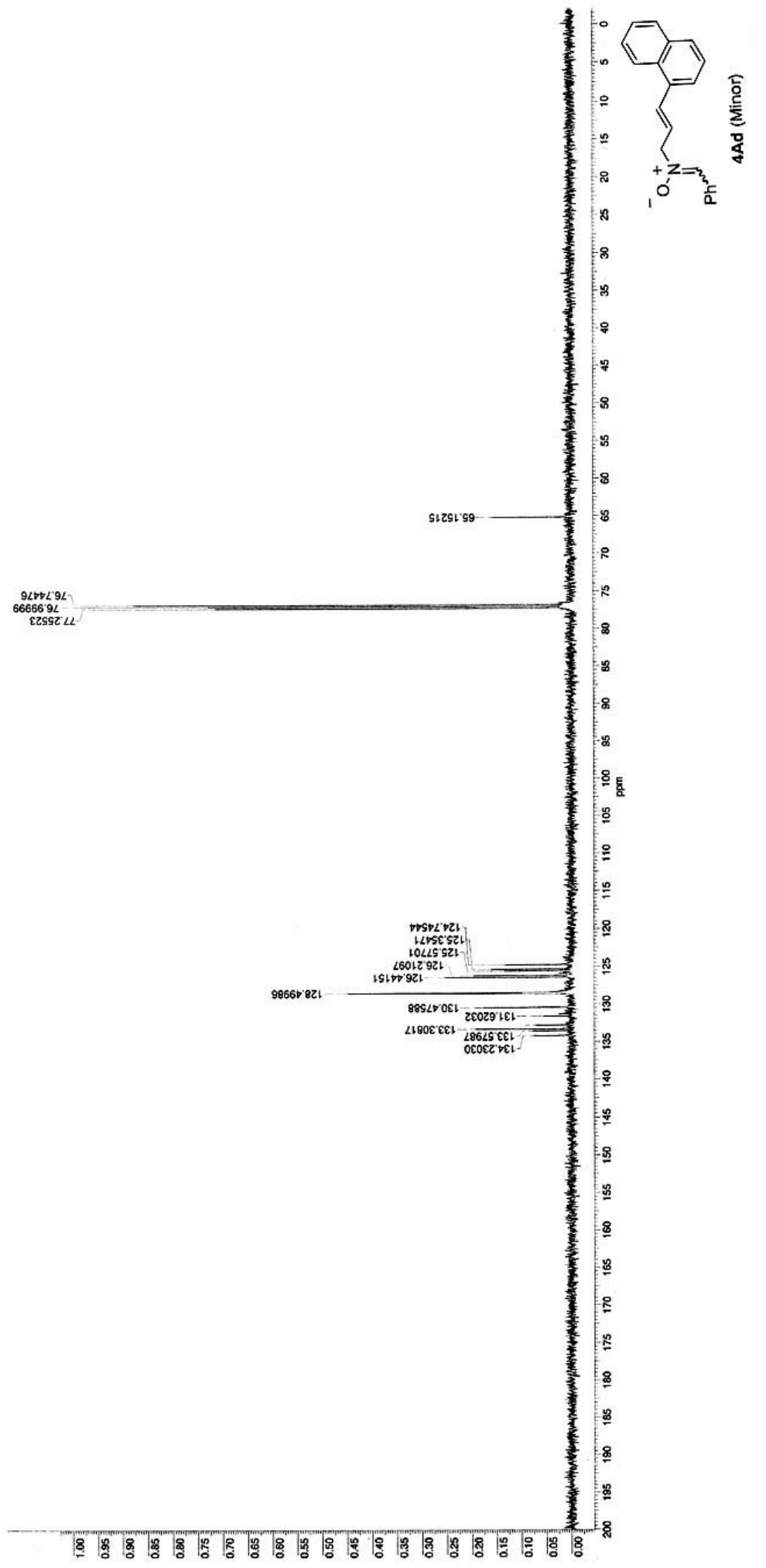




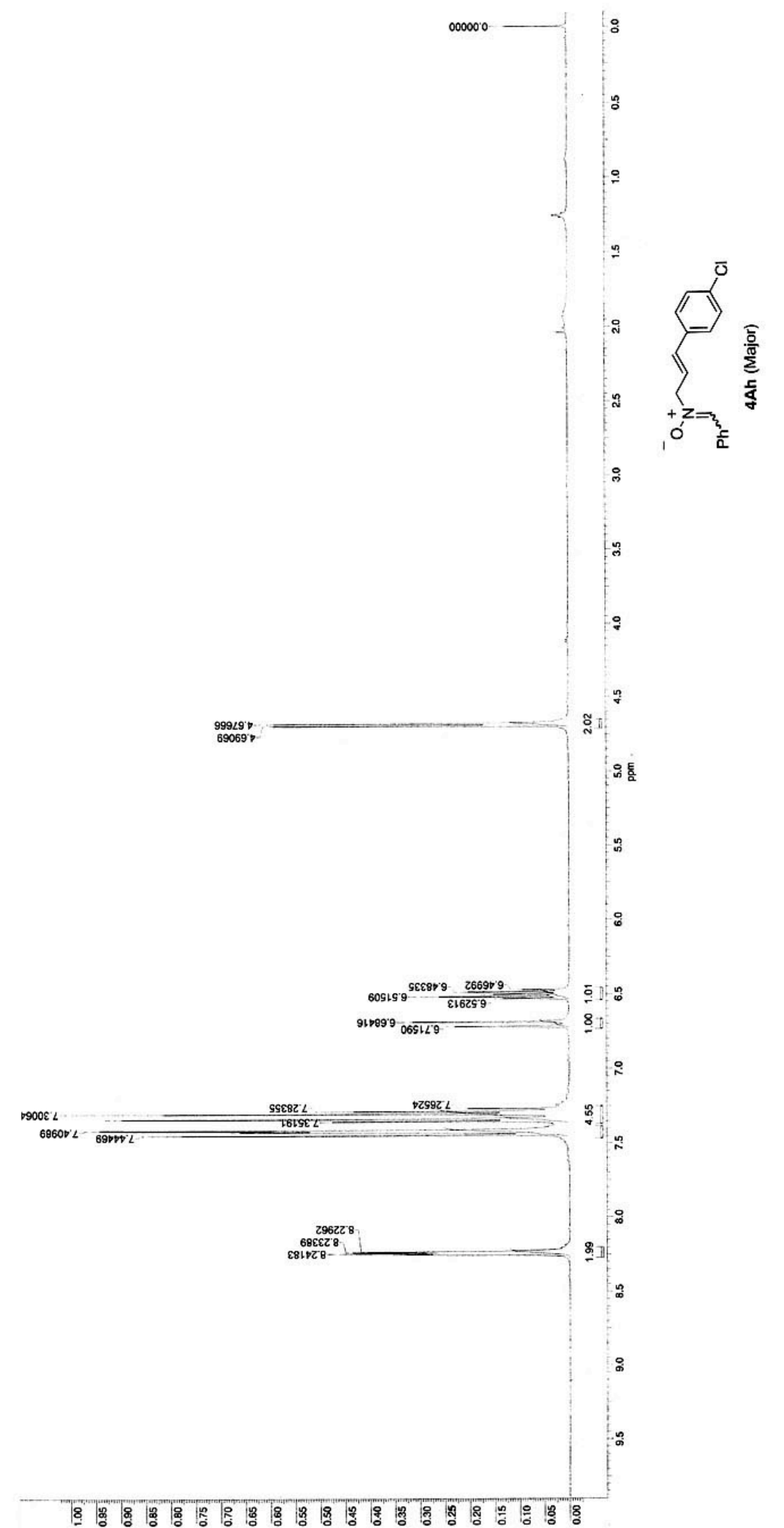




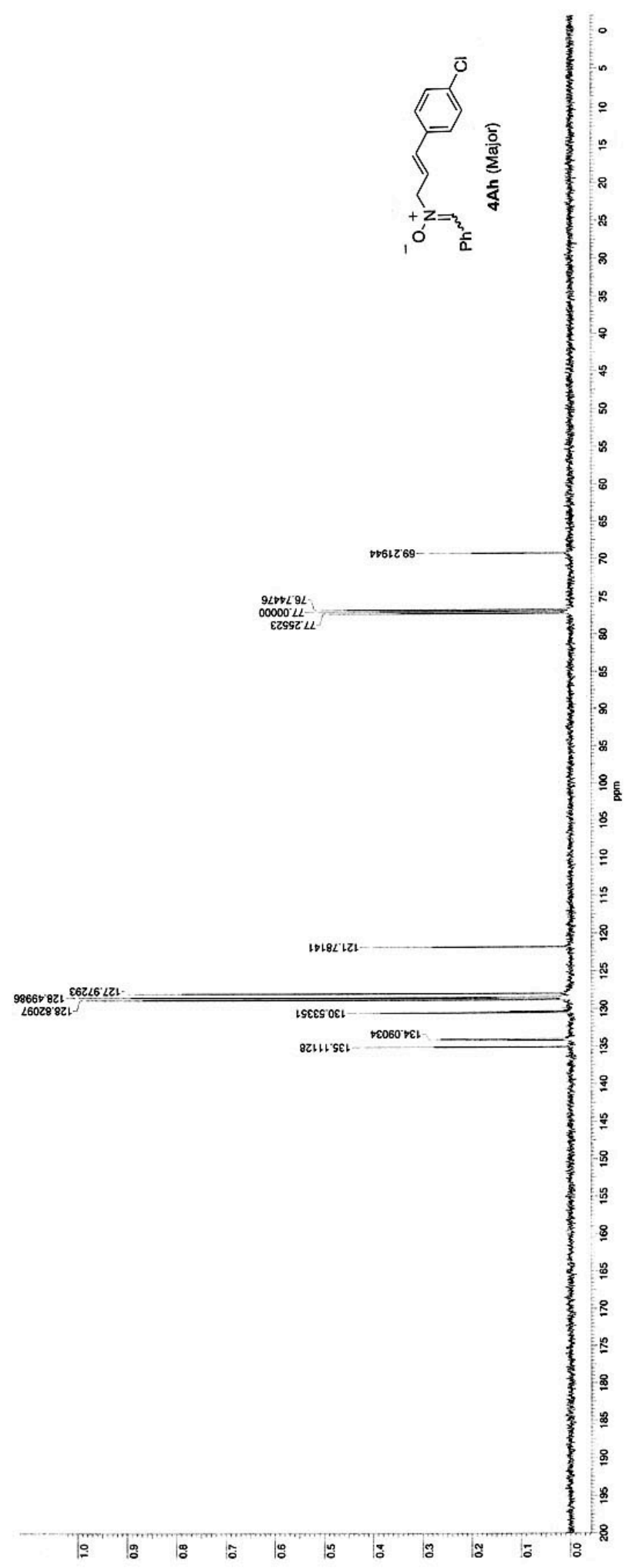




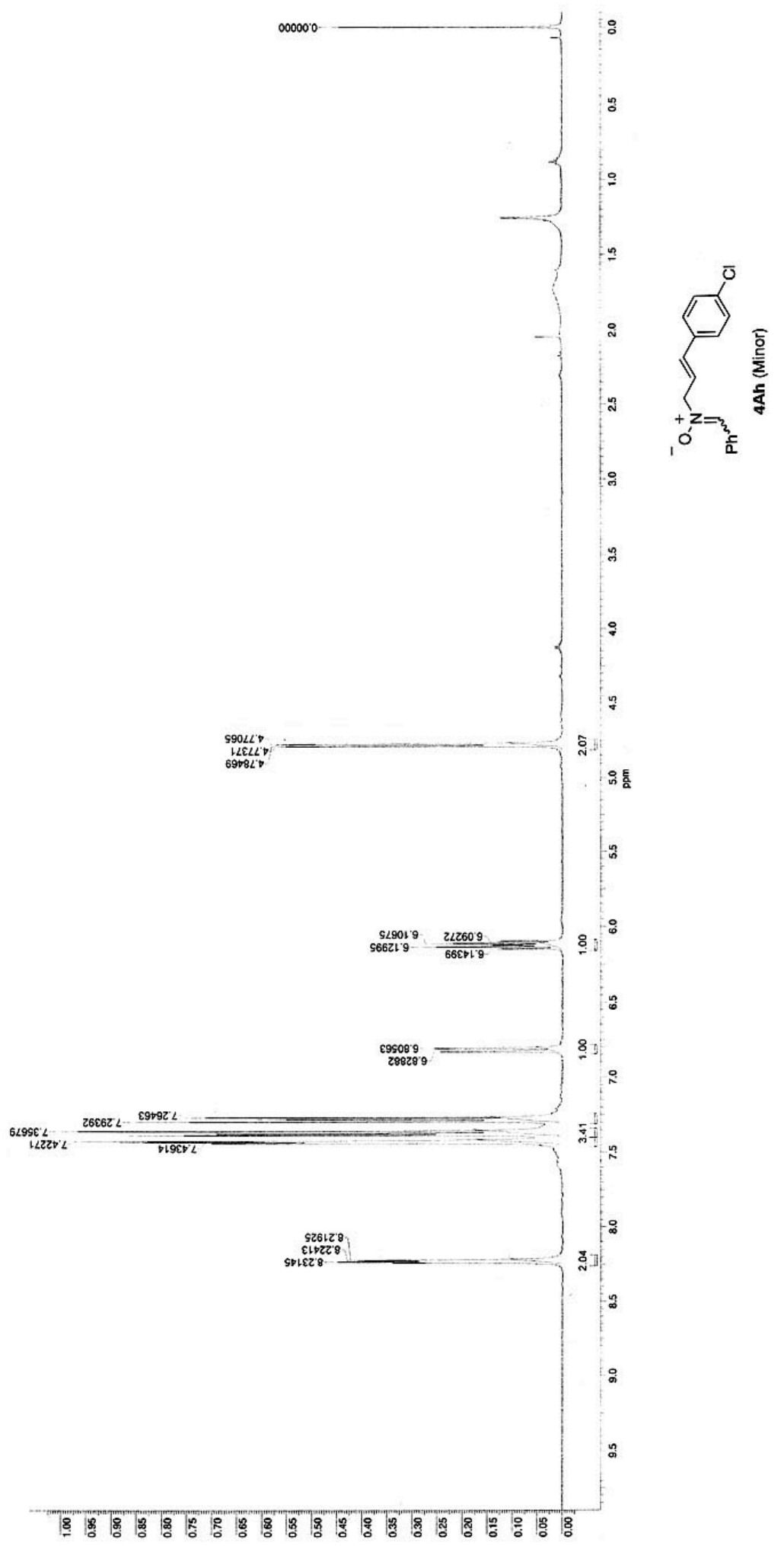




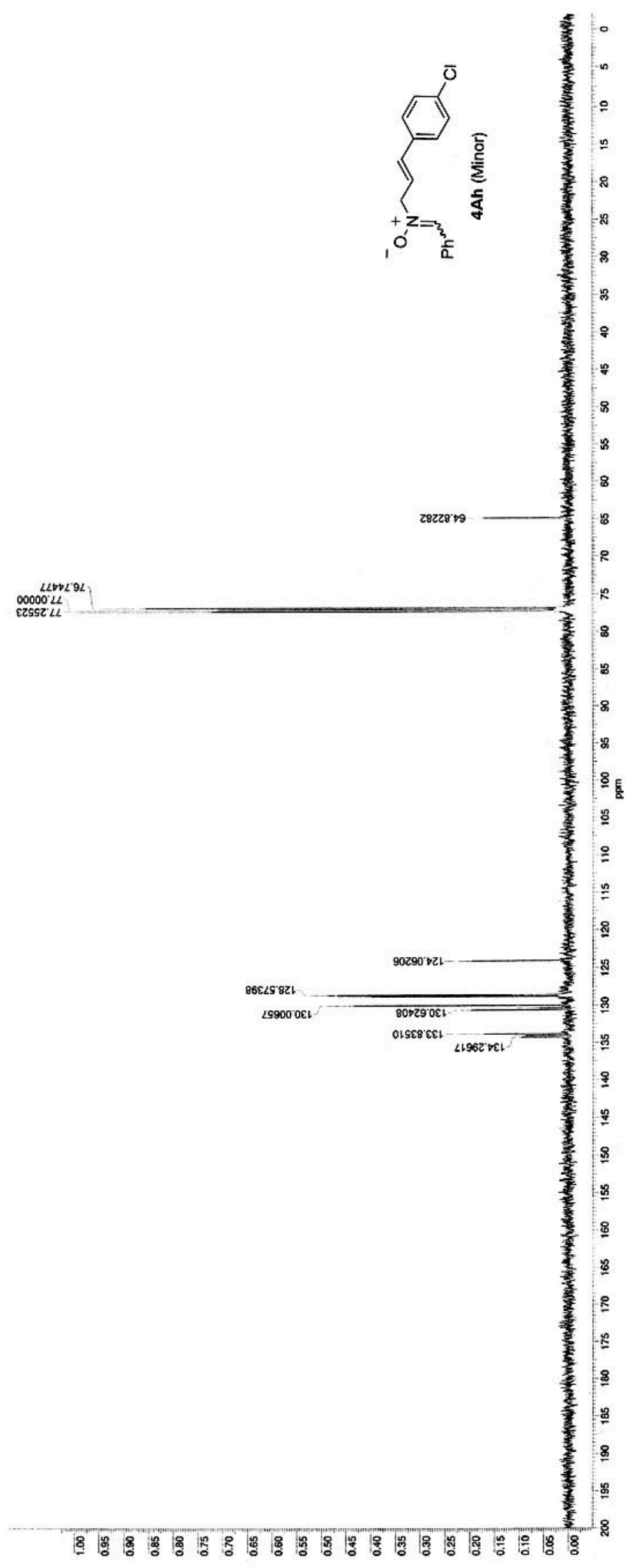




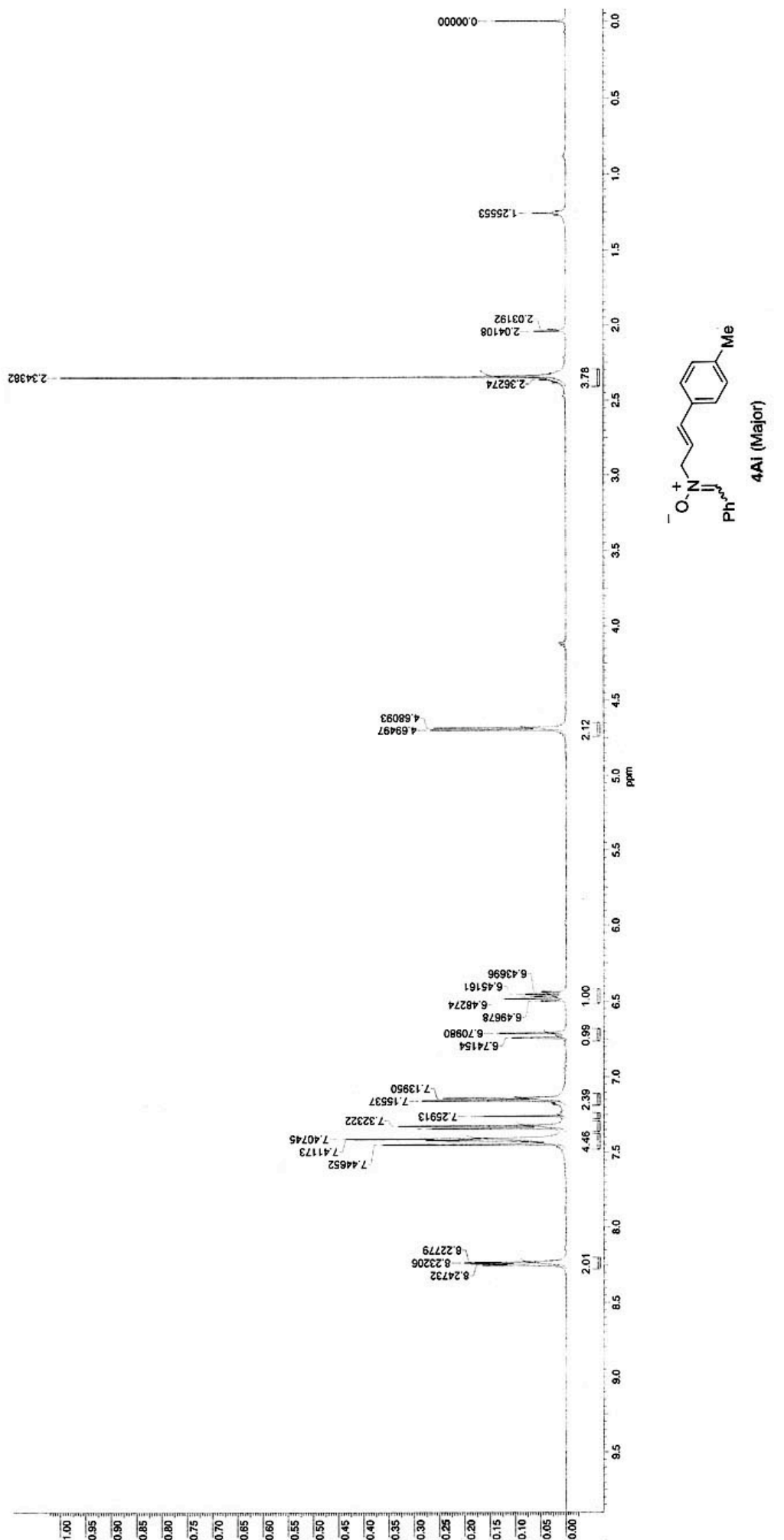




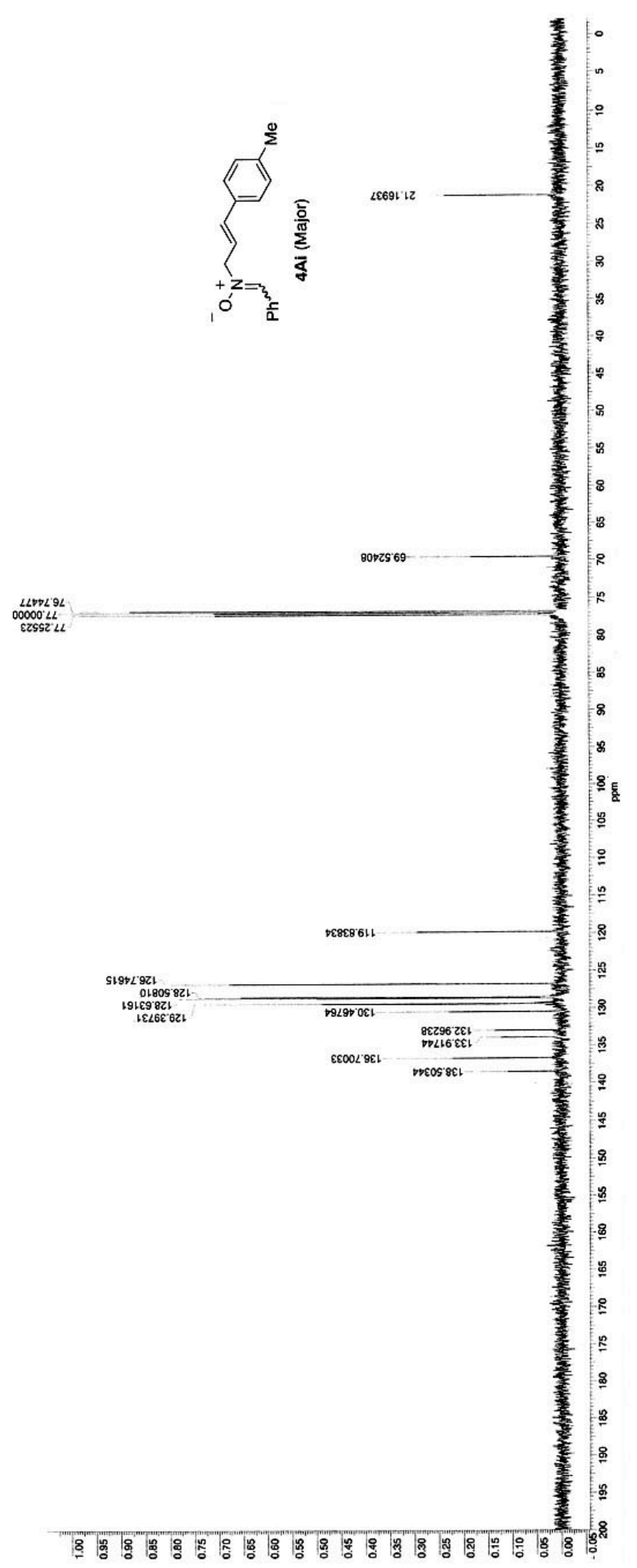




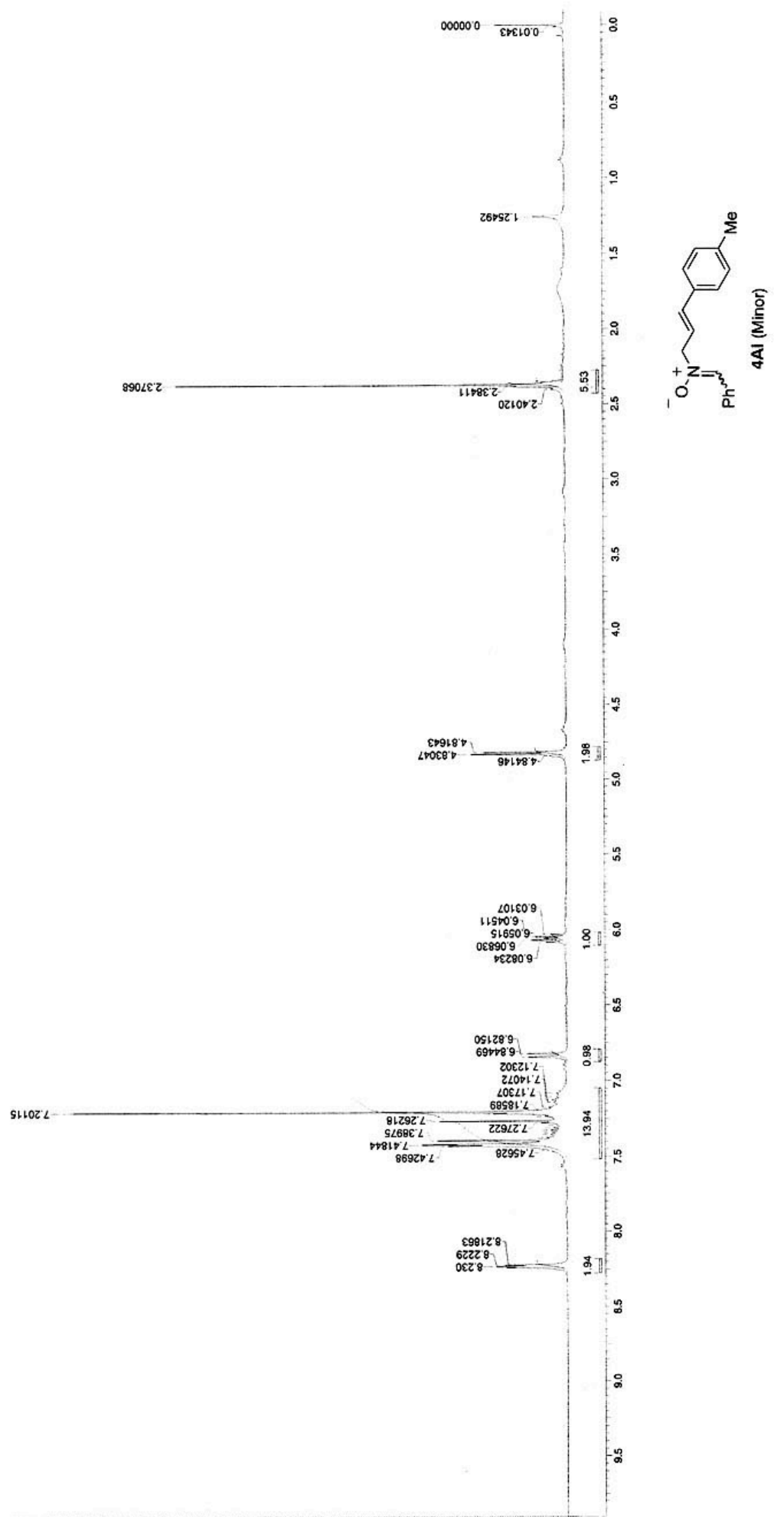

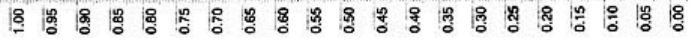




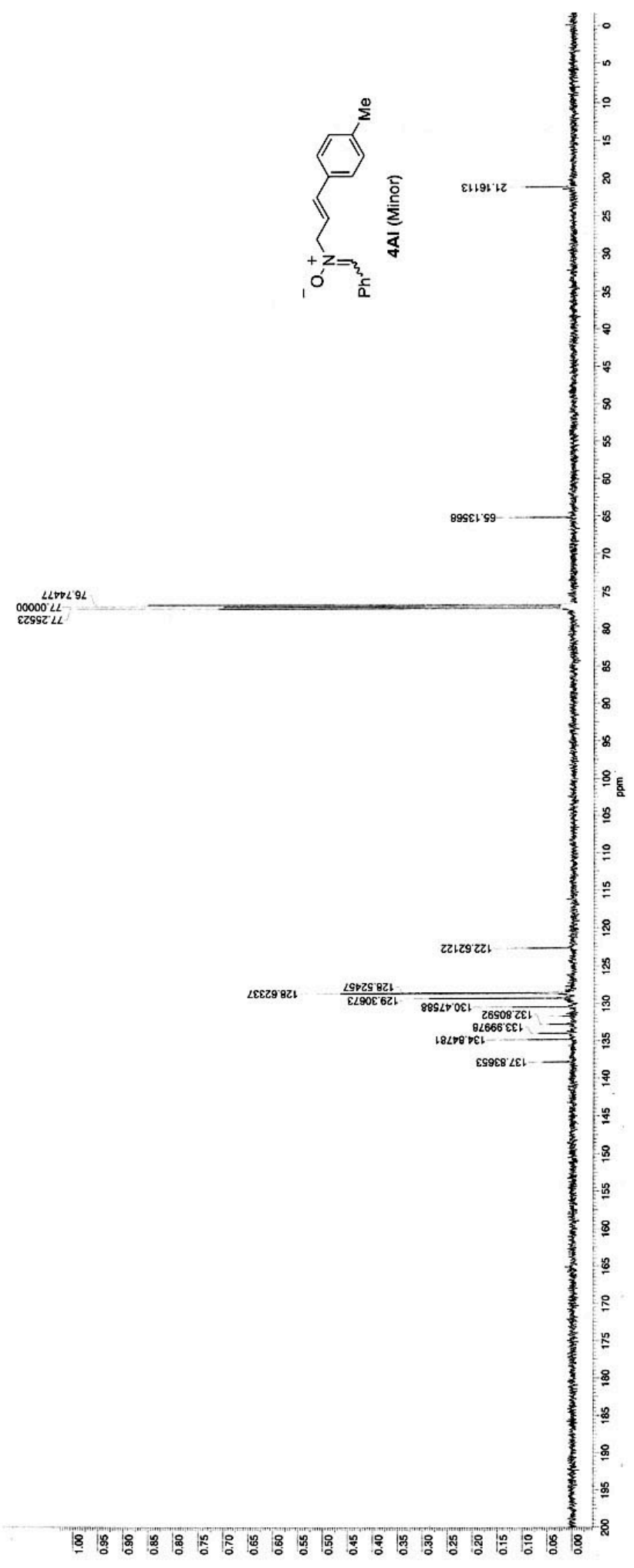




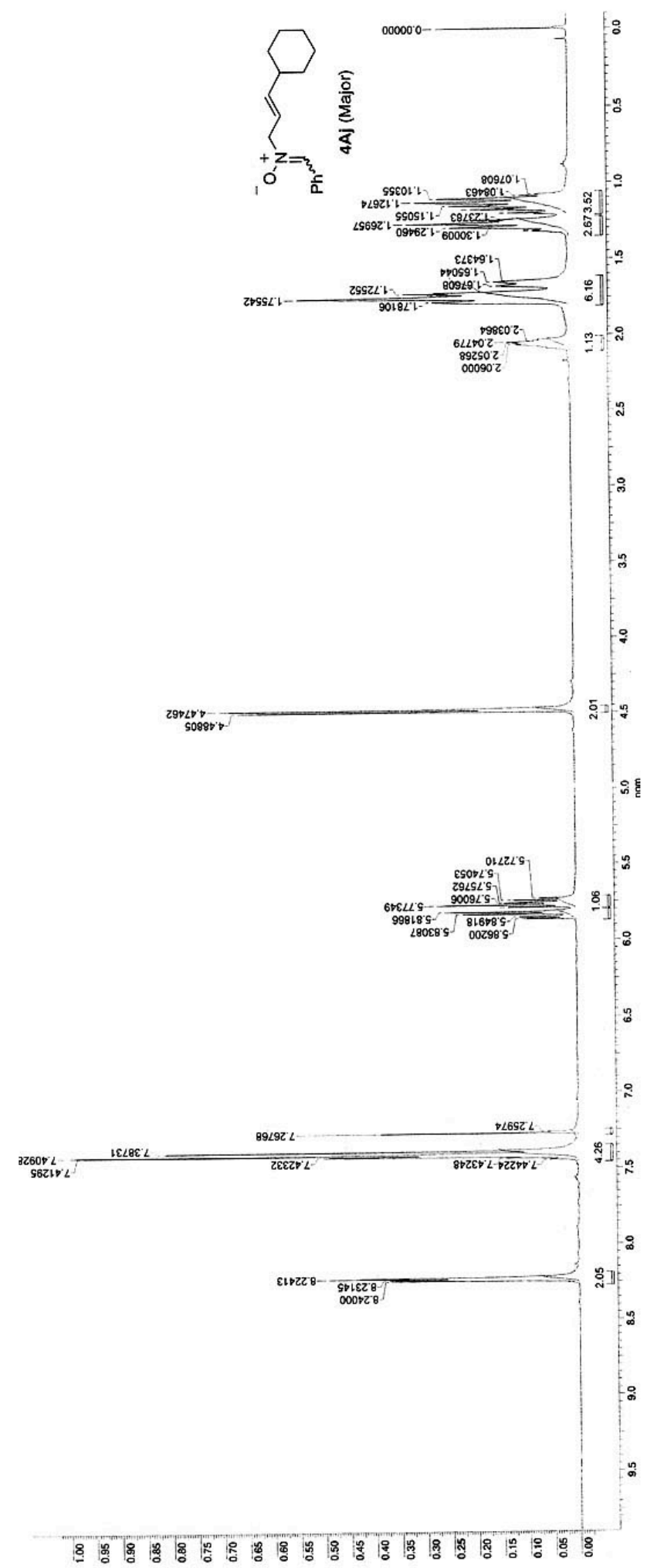




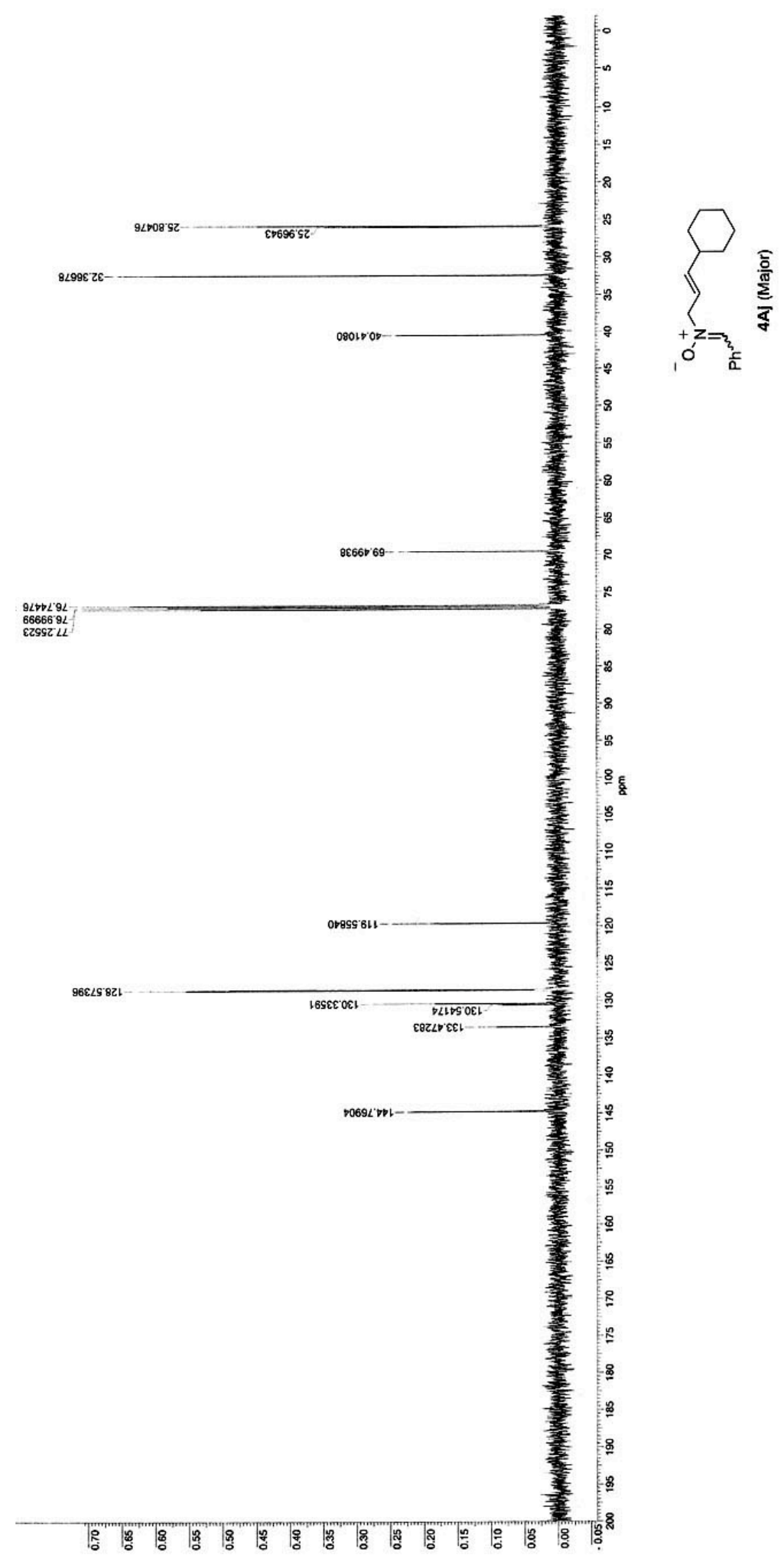




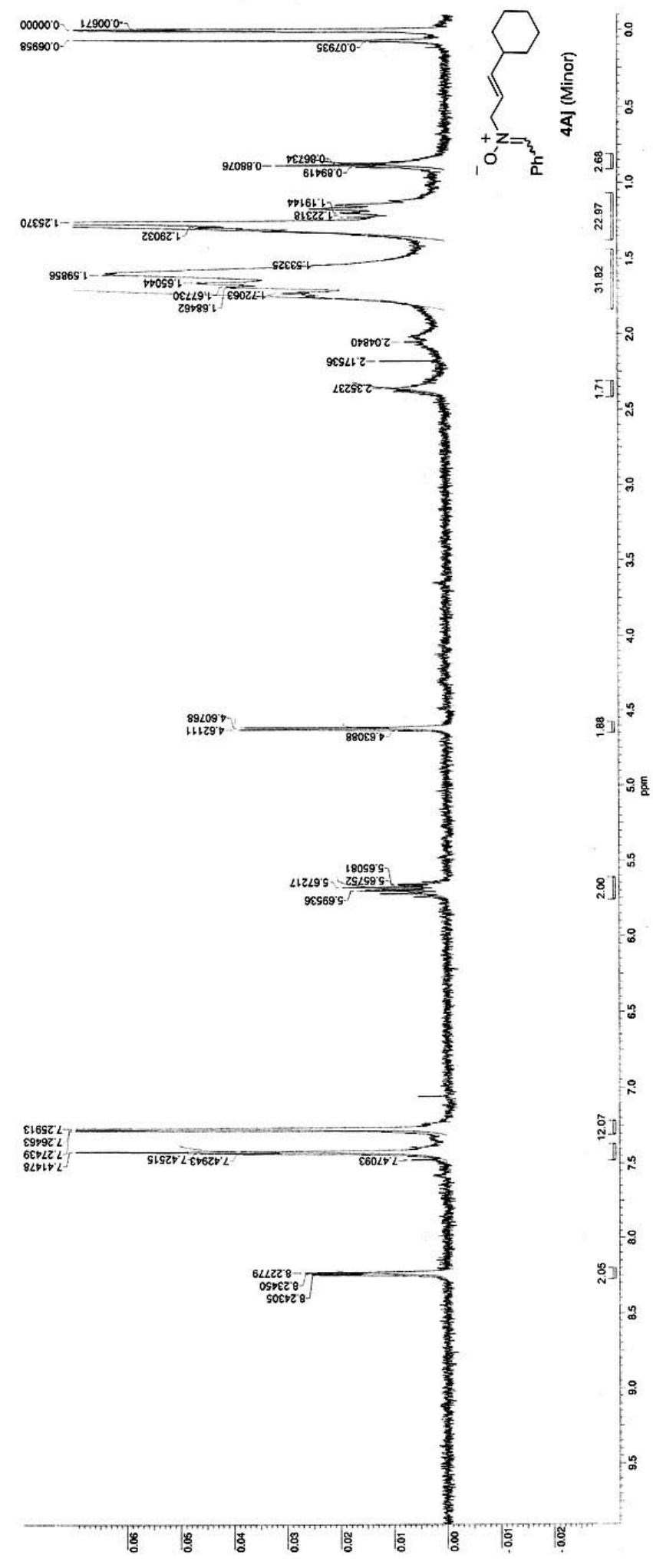




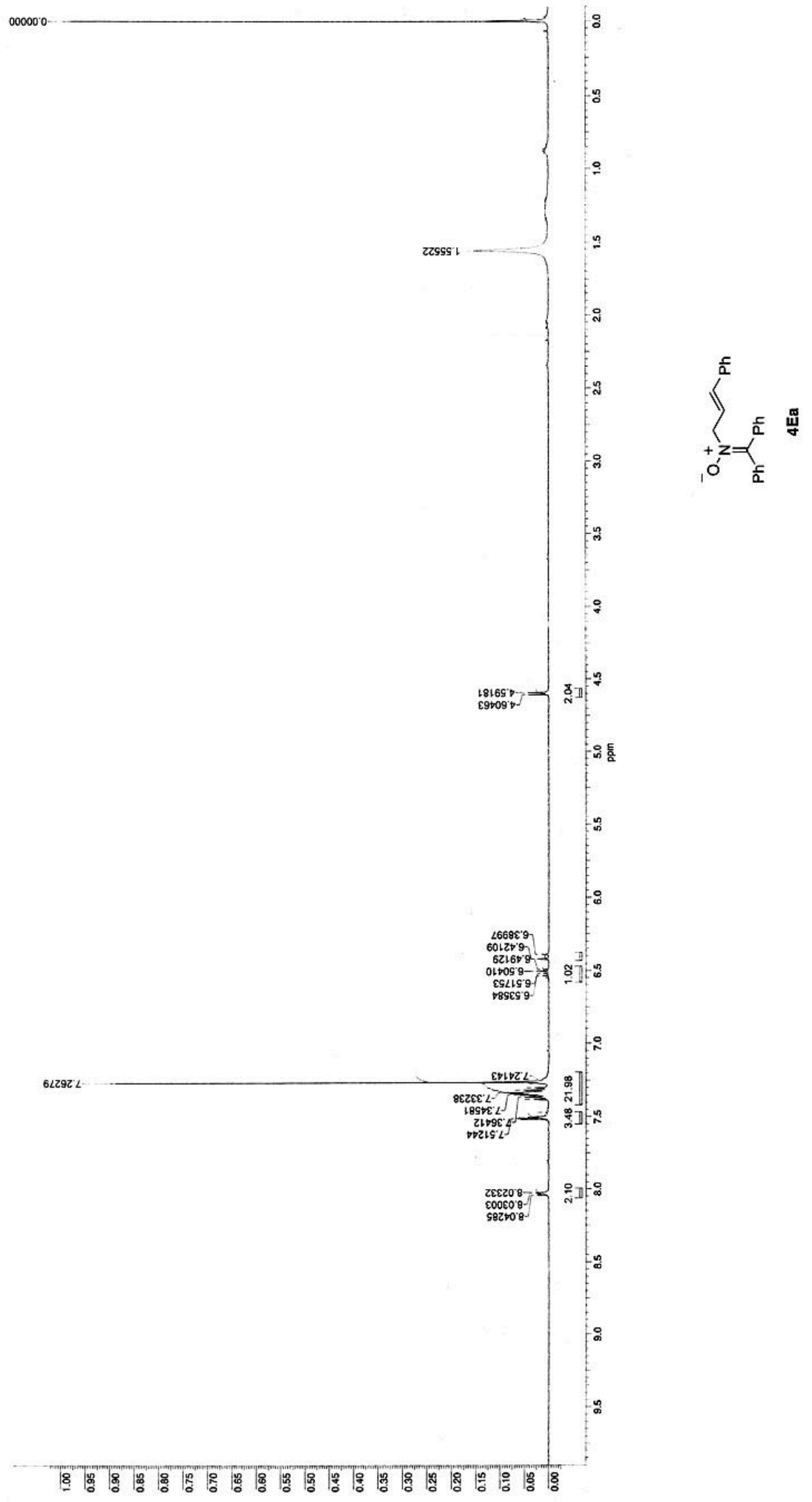




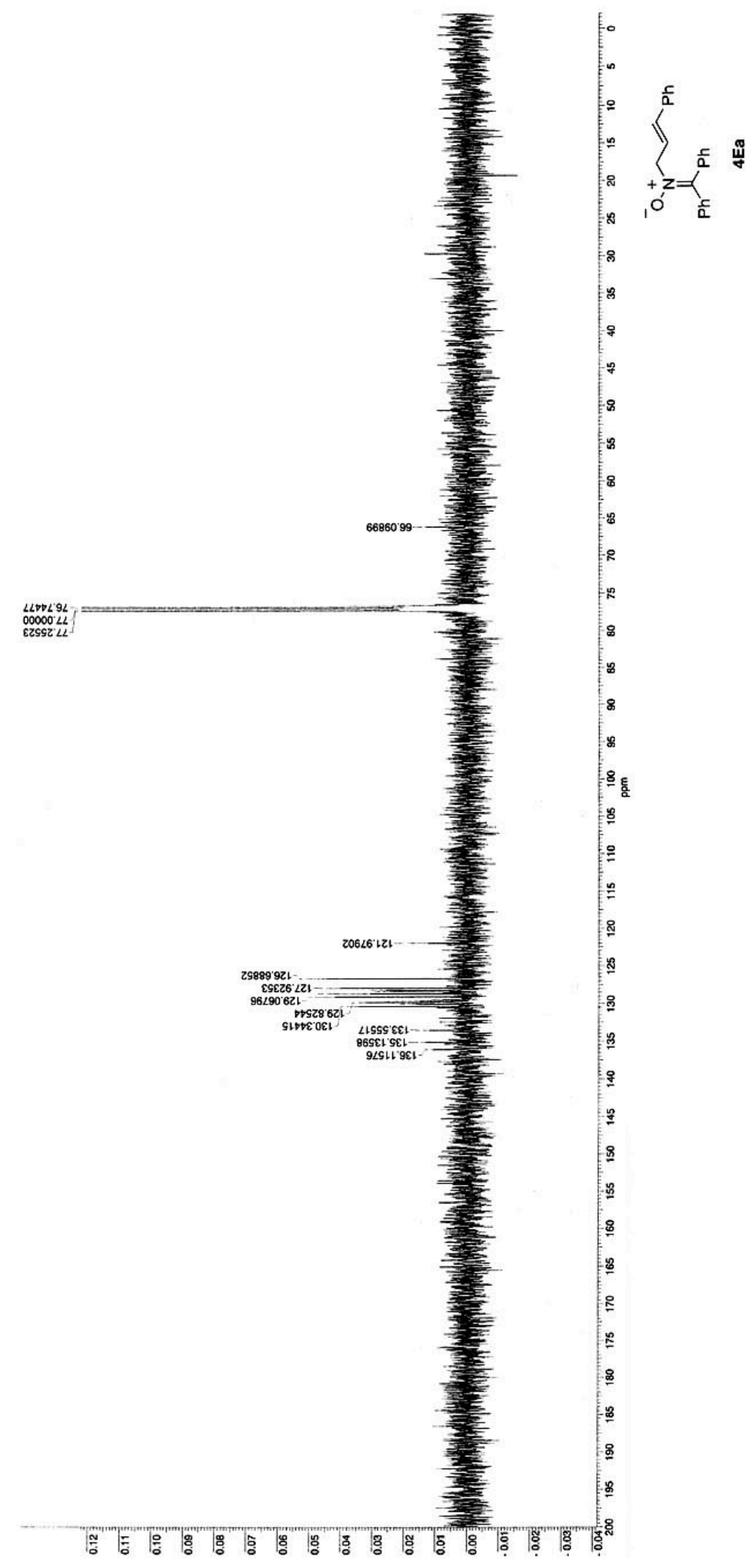




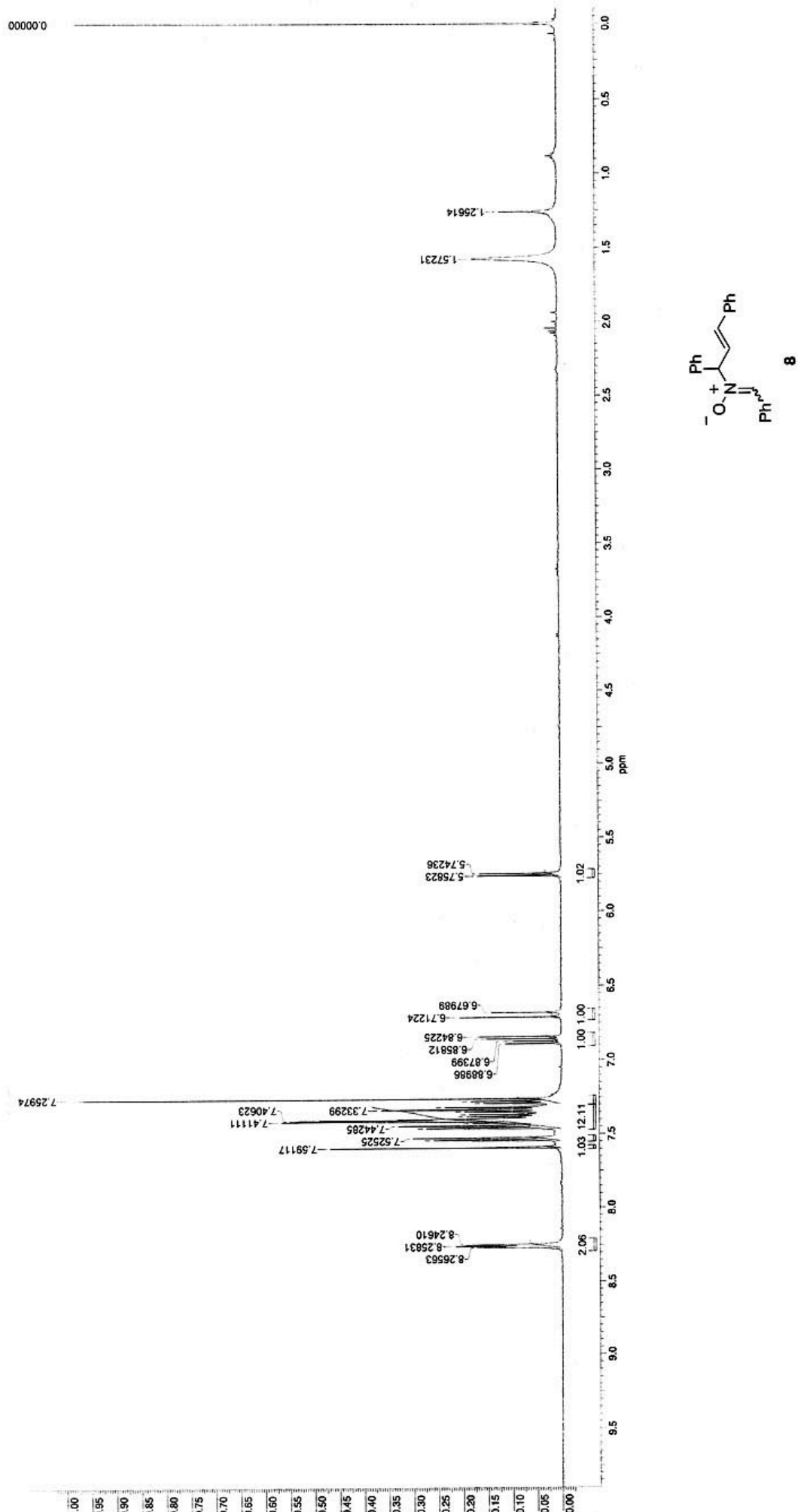

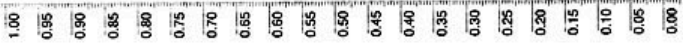




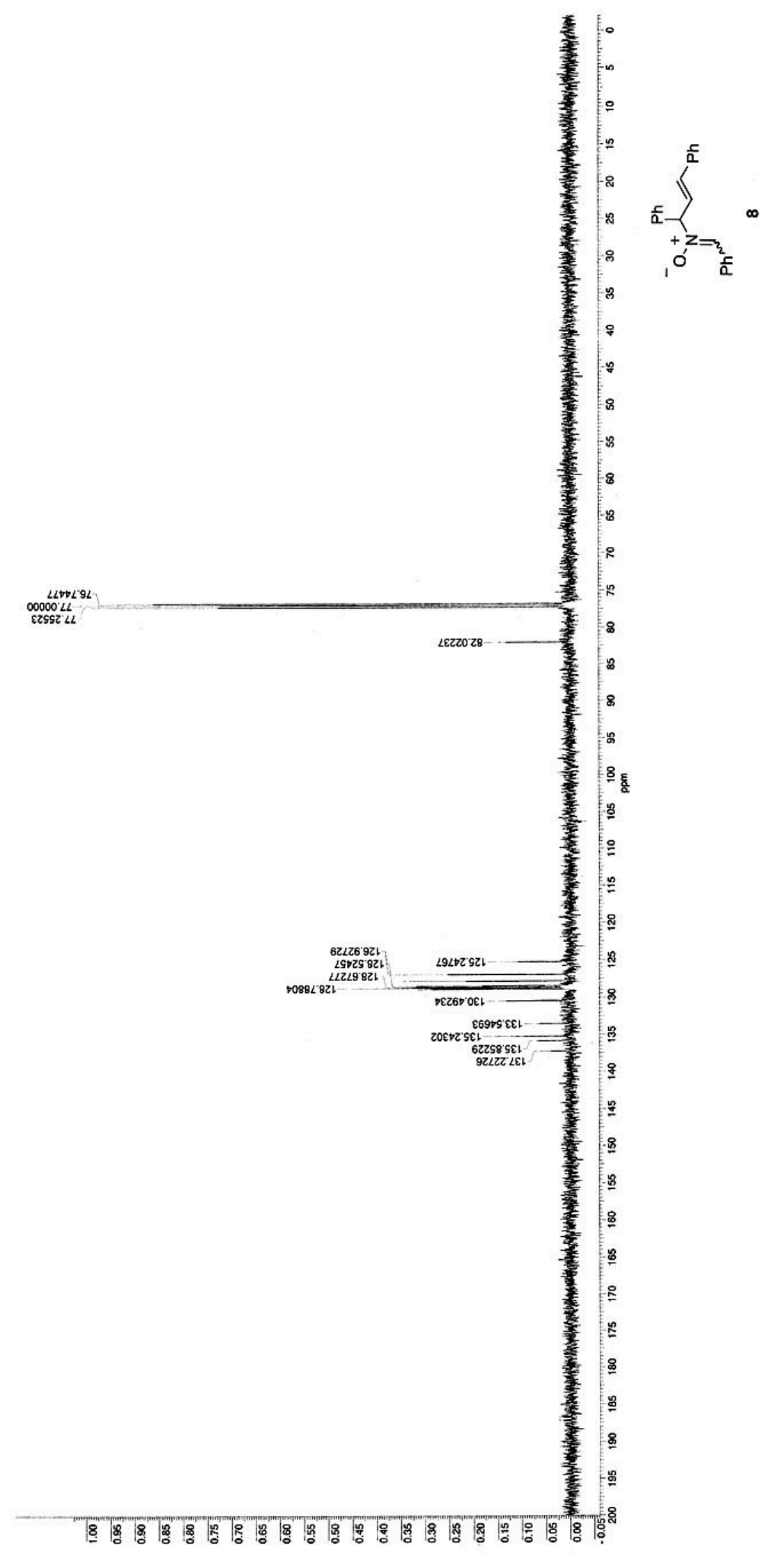




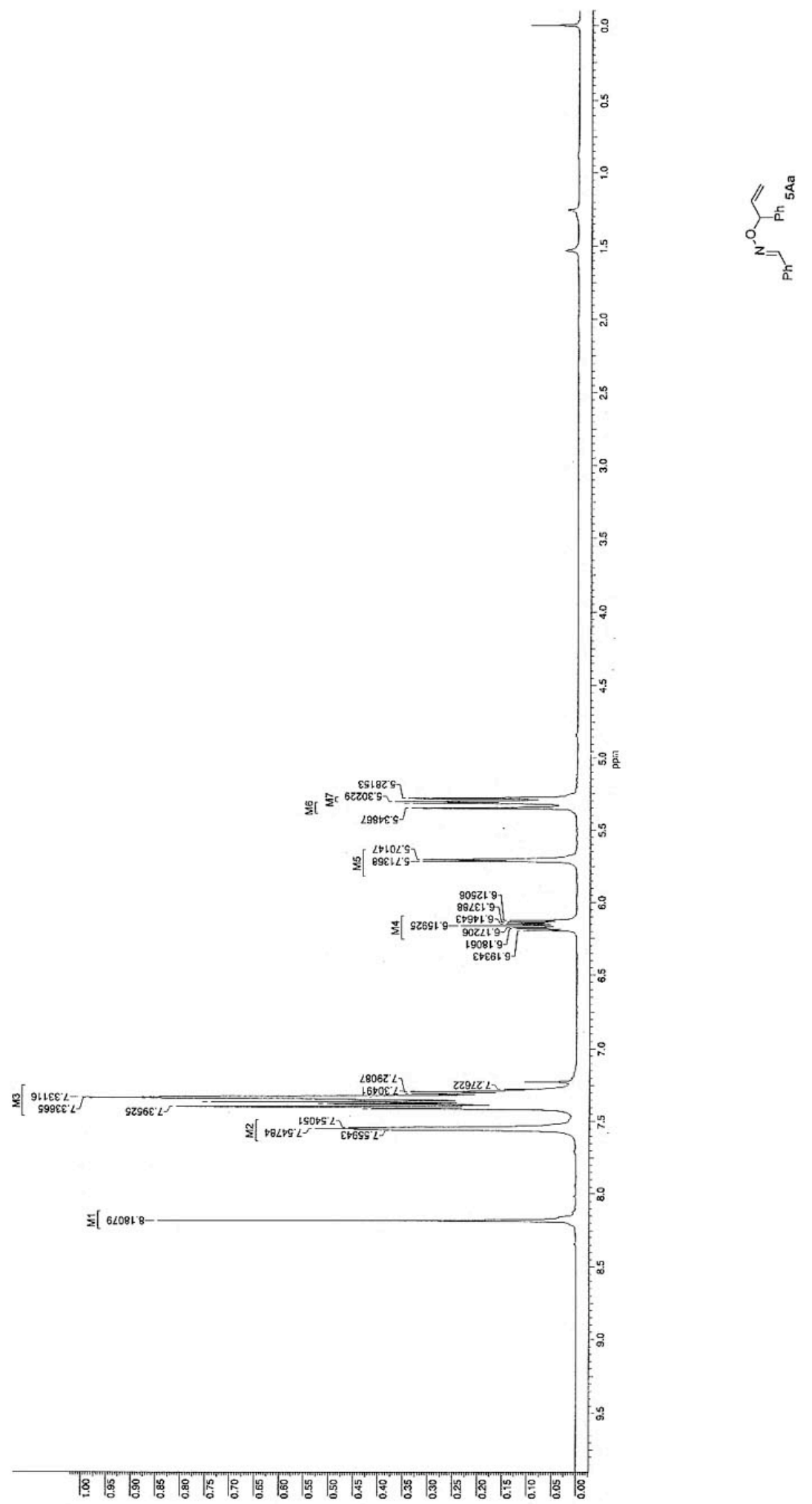




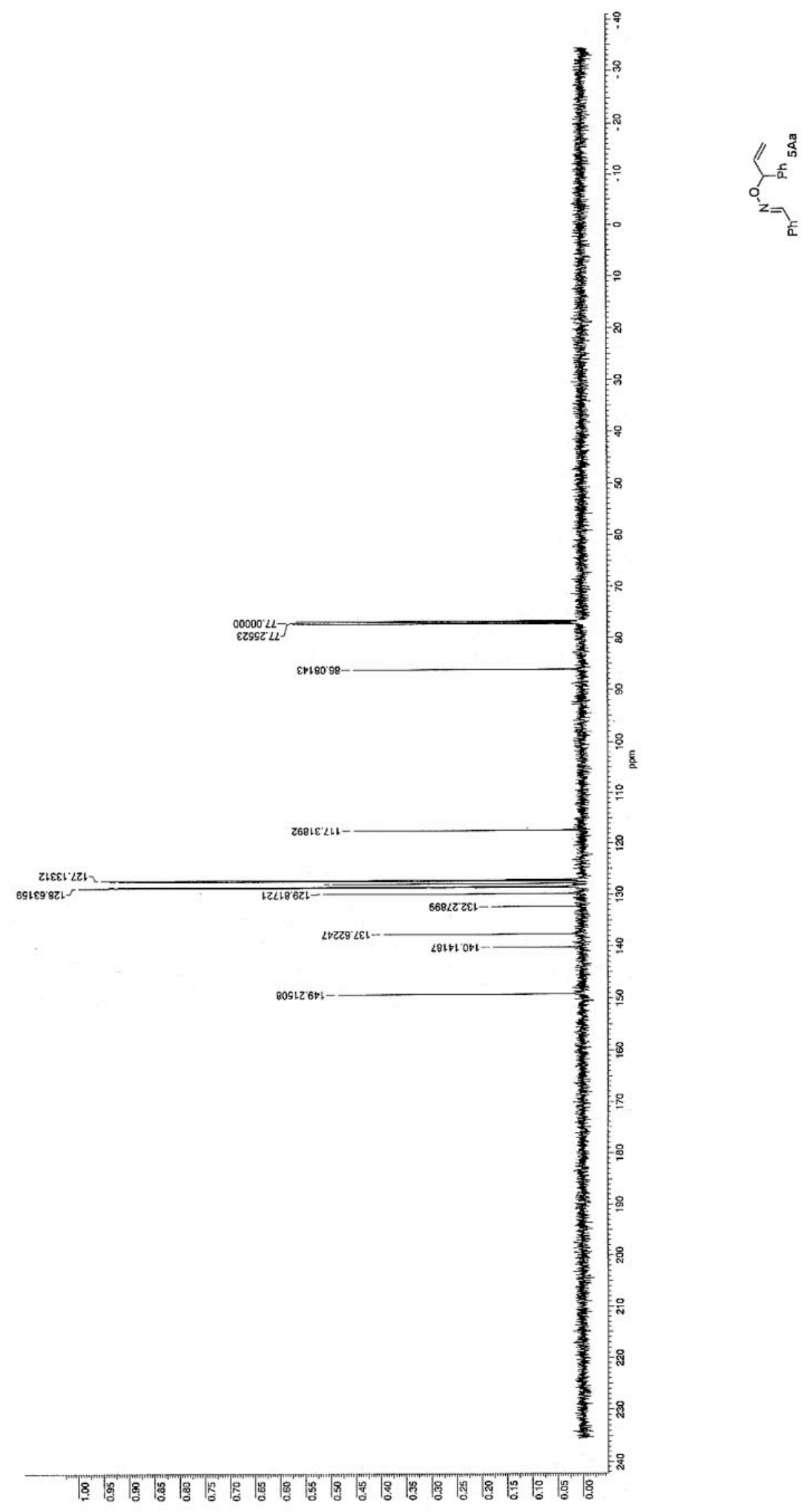

